\title{
THE IMPACT OF ASPECT-ORIENTED PROGRAMMING ON SOFTWARE PERFORMANCE: THE CASE STUDY OF LEADER/FOLLOWERS AND HALF-SYNC/HALF-ASYNC CONCURRENT ARCHITECTURAL PATTERNS
}

by

\author{
Wen-Lin Liu \\ A thesis submitted to the \\ Faculty of Graduate studies and Research \\ In partial fulfillment of \\ The degree requirements of \\ Master of Science in Information System Science \\ Department of Systems and Computer Engineering \\ Carleton University \\ Ottawa, Ontario \\ K1S 5B6 Canada
}

Nov 2010

Copyright $($ Wen-Lin Liu, 2010 


$\begin{array}{ll}\begin{array}{l}\text { Library and Archives } \\ \text { Canada }\end{array} & \begin{array}{l}\text { Bibliotheque et } \\ \text { Archives Canada }\end{array} \\ \begin{array}{l}\text { Published Heritage } \\ \text { Branch }\end{array} & \begin{array}{l}\text { Direction du } \\ \text { Patrimoine de l'édition }\end{array} \\ \begin{array}{l}\text { 395 Wellington Street } \\ \text { Ottawa ON K1A ON4 } \\ \text { Canada }\end{array} & \begin{array}{l}\text { 395, rue Wellington } \\ \text { Ottawa ON K1A ON4 } \\ \text { Canada }\end{array}\end{array}$

Your file Votre référence

ISBN: 978-0-494-79607-8

Our file Notro référence

ISBN: $978-0-494-79607-8$

NOTICE:

The author has granted a nonexclusive license allowing Library and Archives Canada to reproduce, publish, archive, preserve, conserve, communicate to the public by telecommunication or on the Internet, loan, distribute and sell theses worldwide, for commercial or noncommercial purposes, in microform, paper, electronic and/or any other formats.

The author retains copyright ownership and moral rights in this thesis. Neither the thesis nor substantial extracts from it may be printed or otherwise reproduced without the author's permission.
AVIS:

L'auteur a accordé une licence non exclusive permettant à la Bibliothèque et Archives Canada de reproduire, publier, archiver, sauvegarder, conserver, transmettre au public par télécommunication ou par l'Internet, prêter, distribuer et vendre des thèses partout dans le monde, à des fins commerciales ou autres, sur support microforme, papier, électronique et/ou autres formats.

L'auteur conserve la propriété du droit d'auteur et des droits moraux qui protège cette thèse. Ni la thèse ni des extraits substantiels de celle-ci ne doivent être imprimés ou autrement reproduits sans son autorisation.
In compliance with the Canadian Privacy Act some supporting forms may have been removed from this thesis.

While these forms may be included in the document page count, their removal does not represent any loss of content from the thesis.
Conformément à la loi canadienne sur la protection de la vie privée, quelques formulaires secondaires ont été enlevés de cette thèse.

Bien que ces formulaires aient inclus dans la pagination, il n'y aura aucun contenu manquant.

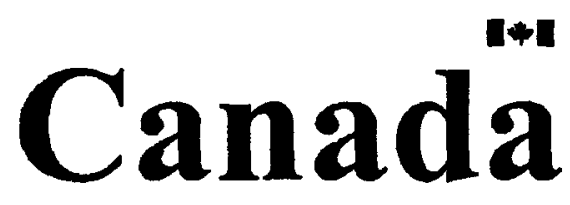




\begin{abstract}
Oftentimes, using Object-Oriented Programming in concurrent systems can leave code tangled and scattered as the complexity of the system increases. The purpose of this study is to investigate the performance impact of using aspect-oriented programming (AOP) while solving addressing the code scattering and tangling issue. Two concurrency architectures, Leader/Followers (LFs) and Half-Sync Half-Async (HS/HA), are used to compare their performance between AOP and non-AOP based program. It is commonly expected that AOP-based program will have lower performance due to the additional overhead that AOP added to the base program. However, the results of this study find out such overhead is very small (negligible), and AOP-based program can even outrun its counterpart. It was discovered the operating system memory management has a role in performance impact. The principal conclusion was that AOP is worthwhile using to mitigate the code scattering and tangling problem, because the AOP-based program's performance is as good as the non-AOP based program.
\end{abstract}




\section{Acknowledgements}

I would like to thanks a lot to my supervisors, Professors Chung-Horng Lung and Samuel A. Ajila, for their wise guidance, patience, funding and continuous support. You both have helped me to find a research topic that I am interested in.

I also owed a grateful gratitude to Xu Zhang, for his comments and supports for providing his tools and codes. I would also like to thank the Faculty of Graduate Studies and Research and NSERC funding this research.

In addition, I am very thankful to my family here in Ottawa, who have been supporting me with all the living expenses and spiritual guidance. Thank you Jacky and Gloria! Finally, I am so grateful to God who always has helped me to meet the right person and providing wise guidance throughout my life. May the world live in peace and let the love of Him live in our heart. 


\section{Table of Contents}

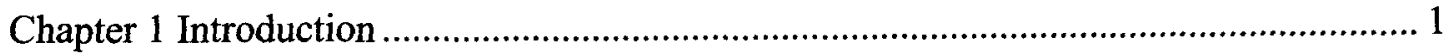

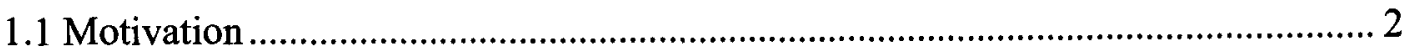



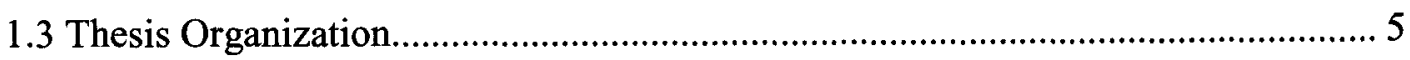

Chapter 2 Background and Literature Review............................................................ 6

2.1 Reverse Engineering ........................................................................................... 6

2.2 Existing Concurrency Software Architecture Patterns............................................ 10

2.2.1 Dynamic thread creation (DTC) .............................................................. 12



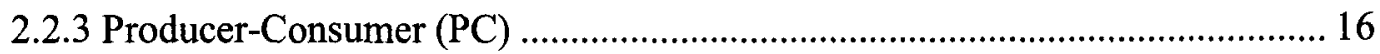

2.2.4 Half-Sync/Half-Async (HS/HA) ………………...................................... 17

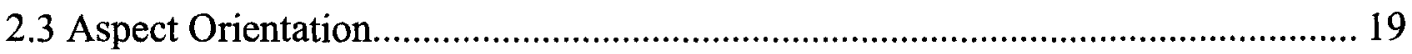



2.3.2 Aspect-Oriented Concerns......................................................................... 21

2.3.3 Reusable Aspect-Oriented Implementation .................................................... 22

2.4 Summary and Scope of the Thesis ................................................................... 24

Chapter 3 Extracting Aspects and Identifying Crosscutting Concerns from the Concurrent

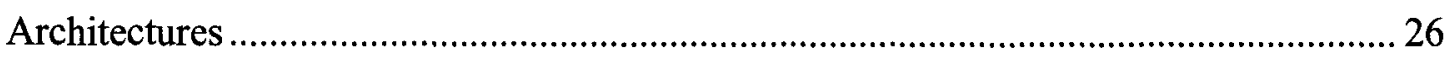

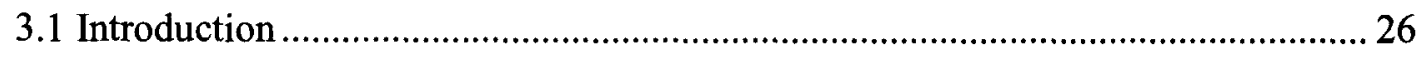

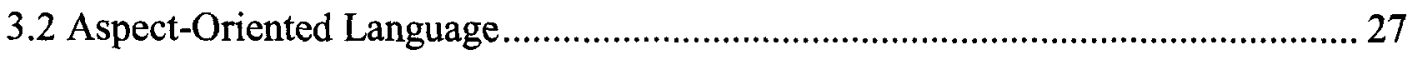



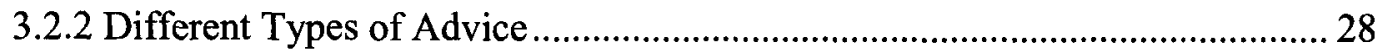

3.3 Extraction of Aspects from LFs Concurrent Architecture ……………………...... 29

3.3.1 Identification of Crosscutting Concerns for LFs ............................................. 32

3.4 Extracting Functional Aspect from HS/HA ……………...................................... 40

3.4.1 Extracting HS/HA Concurrent Architecture................................................... 40

3.4.2 Identifying of Crosscutting Concerns for HS/HA …....................................... 43

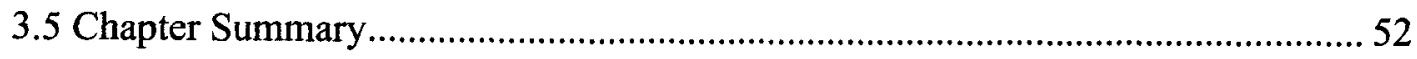


Chapter 4 Experiments and Analysis

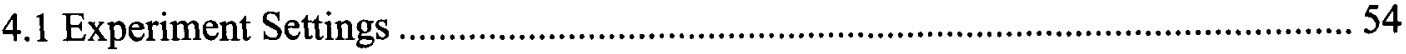

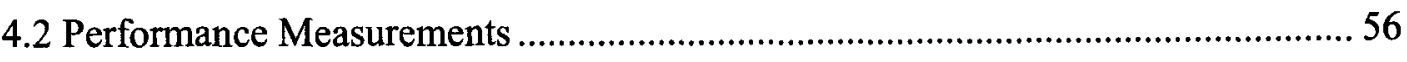

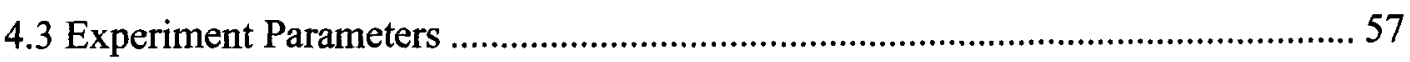

4.4 Performance Evaluation of AOP Using LFs .....................................................5 58

4.4.1 Performance Evaluation of AOP Using LFs with Different Thread Numbers in a CPU-Bound Operating Environment........................................................ 58

4.4.2 Performance Evaluation of AOP Using LFs with Different Number of Threads in a CPU- and $\mathrm{I} / \mathrm{O}-\mathrm{Bound}$ Operating Environment..

4.4.3 Performance Evaluation of AOP Using LFs with Different Number of Join

Points in a CPU-Bound Operating Environment

4.4.4 Performance Evaluation on AOP Using LFs with Different Number of Join

Points in a CPU- and I/O-Bound Operating Environment ............................ 71

4.5 Performance Evaluation on HS/HA

4.5.1 Performance Evaluation on AOP Using HS/HA with Different Number of

Threads in a CPU-Bound Operating Environment.......................................... 76

4.5.2 Performance Evaluation on AOP Using HS/HA with Different Number of

Threads in a CPU- and IO-Bound Operating Environment ........................... 78

4.5.3 Performance Evaluation of AOP Using HS/HA with Different Message Queue

Sizes in a CPU-Bound Operating Environment .......................................... 81

4.5.4 Performance Evaluation of AOP Using HS/HA with Different Message Queue

Size in a CPU- and IO-Bound Operating Environment ............................... 85

4.5.5 Performance Evaluation of HS/HA with Different Number of Join Points in a

CPU-Bound Operating Environment ........................................................ 88

4.5.6 Performance Evaluation of AOP Using HS/HA with Different Number of Join

Points in a CPU- and IO-Bound Operating Environment .............................. 91

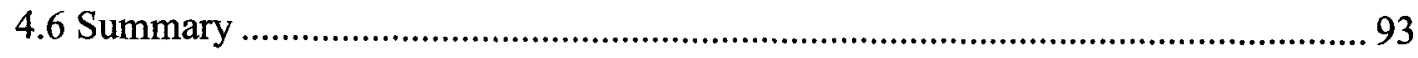

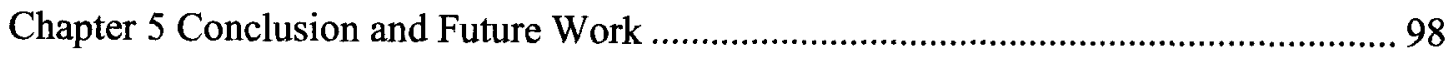

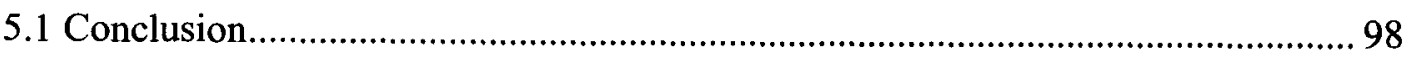




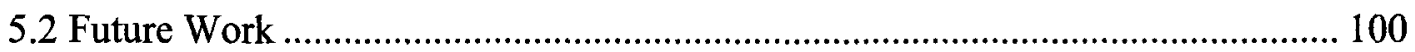

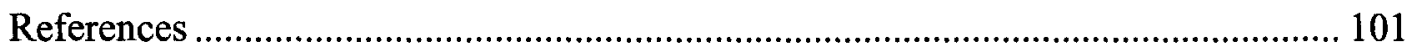

Appendix A Java and AspectJ code for LFs ......................................................... 106

Appendix B Java and AspectJ code for HS/HA .................................................. 112 


\section{List of Figures}

Figure 2.1 Forward, Reverse, and Reengineering [Dem03] ........................................

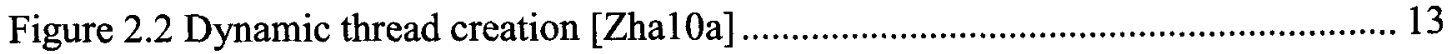

Figure 2.3 Leader/Followers concurrency pattern [Zha10a] ............................................ 15

Figure 2.4 Producer-Consumer problem [Oak04] ....................................................... 16

Figure 2.5 Half-Sync/Half-Async Architecture [Sch96][Zha10a]................................... 18

Figure 2.6 Management of crosscutting concerns with OOP and AOP [Sub05] .............. 20

Figure 2.7 Crosscutting concerns implemented in an application [Lea10] ....................... 22

Figure 2.8 AOP based approach to encapsulate crosscutting concerns into Aspects



Figure 3.1 Class diagram of LFs [Zha10a] ............................................................... 30

Figure 3.2 Modified Class diagram of LFs with aspect module....................................... 33

Figure 3.3 AspectJ code of the aspect class .................................................................... 34

Figure 3.4 Sequence diagram of LFs with Aspect enabled ............................................. 36

Figure 3.5 Sequence diagram of LFs indicating when the advised objects are inactive .. 38

Figure 3.6 Class diagram of HS/HA ........................................................................ 41

Figure 3.7 Class diagram of HS/HA with Aspect enabled ............................................... 44

Figure 3.8 Pointcut description of HS/HA................................................................... 45

Figure 3.9 Advice of HS/HA when writing the message queue ........................................ 46

Figure 3.10 Advice of HS/HA when retrieving the message queue .................................. 47

Figure 3.11 Sequence diagram of HS/HA with Aspect disabled........................................48

Figure 3.12 Sequence diagram of HS/HA with Aspect enabled....................................... 50

Figure 4.1 Emulated multi-tier system of the experiment setup [Zha10a] ....................... 54

Figure 4.2 CPU and cache specification of the server machine ………………………....5

Figure 4.3 Emulated I/O bound operation in request processing [Oso07] [Zha10a]........ 56

Figure 4.4 Average service time of AOP-enabled and AOP-disabled using LFs.............. 59

Figure 4.5 Performance impact of AOP-based program compared with non-AOP based



Figure 4.6 Average service time of LFs with $100 \mathrm{~ms}$ of I/O-bound operation....................64 viii 
Figure 4.7 Performance of AOP-based program compared with the base program 66

Figure 4.8 Average service time of a CPU-bound only operations for AOP-enabled and AOP-disabled programs w.r.t. number of joint points 69

Figure 4.9 Performance impact of the AOP-based program. 70

Figure 4.10 Average service time of a CPU- and IO-bound only operations for AOPenabled and AOP-disabled programs w.r.t. number of joint points. 72

Figure 4.11 Performance of AOP-based program against the base application software 74 Figure 4.12 Average service time of HS/HA with only CPU-bound operation 76

Figure 4.13 Performance impact of AOP-based HS/HA with only CPU-bound operation 78

Figure 4.14 Average service time of HS/HA with CPU- and IO-bound operation 80

Figure 4.15 Performance impact of AOP-based HS/HA with CPU- and IO-bound operation 81

Figure 4.16 Average service time of AOP-enabled and AOP-disabled using HS/HA with only CPU-bound operations. 83

Figure 4.17 Performance impact of AOP-based HS/HA with only CPU-bound operation 84

Figure 4.18 Average service time of HS/HA with CPU- and IO-Bound database operation 86

Figure 4.19 Performance impact of AOP-based HS/HA with CPU- and IO-bound operation. 87

Figure 4.20 Average service time of a CPU-bound only operations for AOP-enabled and AOP-disabled programs w.r.t. number of joint points 89

Figure 4.21 Performance impact of HS/HA AOP-based program 90

Figure 4.22 Average service time of a CPU- and IO-Bound operations for AOP-enabled and AOP-disabled programs w.r.t. number of joint points . 92

Figure 4.23 Performance impact of HS/HA with CPU- and IO-bound operation............ 93 Figure 4.24 Average service time of LFs and HS/Ha with CPU-bound only operations 95 Figure 4.25 Performance impact of LFs and HS/HA with CPU-bound operation .95 
Figure 4.26 Average service time of LFs and HS/HA with CPU- and IO-bound operations 96

Figure 4.27 Performance impact of LFs and HS/HA with CPU- and IO-bound operation 


\section{List of Tables}

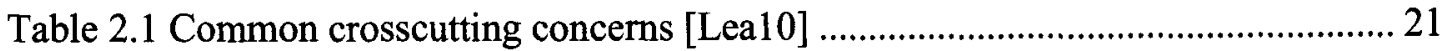

Table 3.1 Terminology of AspectJ [Gra03][Spi10][Cha03] ........................................28

Table 3.2 Different types of advice [Gra03][Spi10][Mil05] ..........................................29

Table 4.1 Average service time of with different thread numbers created .......................6 60

Table 4.2 Cache fault/second with different number of threads created .........................61

Table 4.3 Average service time with different thread number created...........................65

Table 4.4 Cache fault/second of LFs with different number of threads created under CPU-

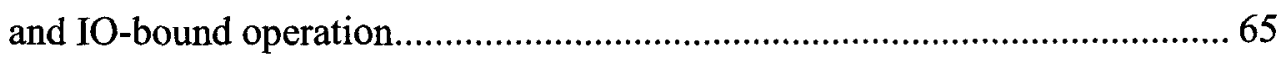

Table 4.5 Cache fault/second of LFs with different number of threads created under CPU-

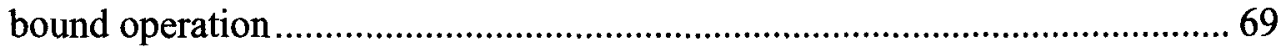

Table 4.6 Cache fault/second of LFs with different number of threads created under CPU-

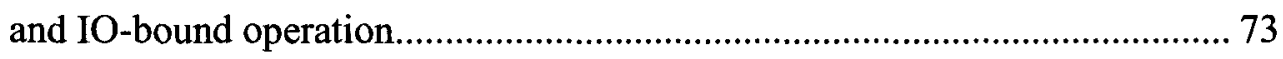

Table 4.7 Cache fault/second of HS/HA with only CPU-bound operation ..................... 77

Table 4.8 Cache fault/second of HS/HA with CPU- and IO-bound operating environment

Table 4.9 Cache fault/second of HS/HA with only CPU operating environment ............ 83

Table 4.10 Cache fault/second of HS/HA with CPU- and IO-Bound operating

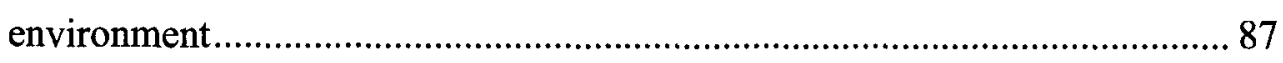

Table 4.11 Cache fault/second of HS/HA with CPU-bound operating environment ....... 90

Table 4.12 Cache fault/second of HS/HA with CPU- and IO-Bound operating environment. 


\section{Chapter 1 Introduction}

The evolution of new theories and technologies in software engineering over the past years has opened up the path for more complex computer systems and large scale requirements.

Constructing quality software within deadline, budget and other constraints specified by customers requires highly experienced personnel with a systematic, disciplined and quantifiable approach to the development, operation, and maintenance of software. Such complex procedures during the course of developing software usually end up with missing requirements due to the time-frame, budget, or other factors such as inexperienced software developers. Software is generally more complete and robust as the software engineer becomes more experienced. However, software technologies are evolving quickly and it is challenging for software engineers to support software evolution efficiently. In the real word scenario, a software development process can undergo several versions before it becomes mature and robust, like patches and updates for an upgraded version. Most of the time, upgrading the software is necessary because of missing requirements during the specification phase or simply in response to the rapidly evolving technologies.

The traditional way of updating the source program can interfere with the existing software which may consist of multiple layers. Potential changes in the code could also deviate from the original behavior of the software, causing unexpected bugs. When the 
source code is modified to suit new requirements, the integrity of the original program is usually compromised and the software structure often drifts, leading the maintenance of the software to become more challenging over time. As noted, the changes or addition of more functionality to the software system can influence the original cohesiveness [Aok07]. Consequently, more work is needed to maintain the software. If the requirements crosscut several functionalities, this will lead to scattered code across the program, which means all the functionalities involved will be modified and the effect will be huge. As a result, subsystems lose their cohesion and become highly coupled instead. The program also becomes more difficult to understand and harder to maintain.

\subsection{Motivation}

The scattered and tangled code problem can be solved by using Aspect-Oriented Programming (AOP) or Aspect-Oriented Design (AOD). AOP can help developers to deal with scattering and tangling of concerns populated in the functionalities of the software. Concerns are the system requirements or features. These concerns of the same nature will be encapsulated into an aspect by using AOP technologies. Thus, the code consisting of the concern is centralized at the same place, called advice. An advice is a piece of code invoked in order to fulfill the specific requirement. However, the base program is oblivious to the invocation of the advice. The invocation of advice is determined by the description of pointcut. Pointcut is a description for a group of join points for inclusion of an advice. 
The base program has no idea of the existence of such concerns, and is not aware of the transfer of the control flow from the base program to the aspect code (advice) when the concern of a specific requirement needs to be fulfilled. The moving of control flow between the base program and advices causes execution overhead. However, the performance impact of the overhead AOP has not yet been reported in the literature, especially for distributed and concurrent systems. Thus, we hypothesize that adding aspects will affect the performance of the base program due to the overhead caused by switching control flow.

The approach in this thesis is to extract aspects from the functionalities of the existing well-known concurrency architectures, Half-Sync/Half-Async (HS/HA) and Leader/Followers (LFs) [Zha10a][Lun06]. The extracted aspects will be analyzed and those aspects which cut across different concerns will be identified. The concerns found mostly in the concurrency architectures are related to the synchronization of critical sections under the multi-threaded environment. Those concerns are to be identified and encapsulated into advices to increase system's maintainability.

The modified program with concerns that are abstracted and encapsulated using AOP has the same functionalities and fulfills the same requirements as the base program. However, the performance overhead needs to be carefully identified and studied to better understand the potential impact of AOP. Therefore, the motivation is that knowing the performance impact by using AOP will give developers a better idea of what AOP can contribute to the overhead of switching the control flow back and forth. Therefore, 
software developers can have a better view of the various quality attributes when using AOD and AOP.

\subsection{Contributions}

The main contributions are the applications of AOD/AOP to complex concurrency architectures and the study of the performance impact of using AOP. Specifically, the contributions of this thesis are as follows:

- Two well-known concurrency architectures (HS/HA and LFs) are studied and crosscutting and tangling concerns of HS/HA and LFs are identified, extracted and encapsulated into advices using AOD and AOP.

- Performance impact of the overhead caused by AOP is measured.

- Propose a way to measure the performance impact of AOP by manually adding method calls which are to be captured as join points. These method calls have no relation with the functionalities or requirements of the program. Additionally, the performance impact of aspect enabled and disabled programs with method calls added are analyzed. These method calls become join points if the aspect code is enabled. If the aspect is disabled, these method calls are just regular method calls.

The research provides useful knowledge to support the evolution of concurrency architectures and presents practical information of performance impact of AOP. 


\subsection{Thesis Organization}

This thesis is organized as follows: Chapter 2 describes the background knowledge and related work on the topic of concurrency and performance analysis using AOP. Chapter 3 describes the approach and the technique of identifying the functionalities that are scattered or tangled in different parts of the software, and discusses what issues are involved in using such techniques from the performance point of view. Chapter 4 presents the analysis and the comparison between the non-aspect woven program and aspect woven program, and discusses the results from the performance point of view. Chapter 5 presents the conclusions and future work. 


\section{Chapter 2 Background and Literature Review}

This chapter provides the relevant background information and literature review on software engineering, different existing concurrency software architecture, and the current state of research on aspect-oriented programming.

\subsection{Reverse Engineering}

Due to the rapid changes of the growing technologies, the software product may undergo a process of adding or removing services to suit new requirements in the real world market. Thus, reverse engineering (RE) emerged in the 90s to suit these needs, in order to use what was already deployed in the market (legacy system), analyze its system and software structures, functionalities and operations. The major purpose of using RE is to deduce what has already been done in the past, and verify whether we can reuse the parts or not, so as not to reinvent the wheels and boost up the development time [Wik10a]. This appears as going backwards to the development cycle [War92] as shown in Figure 2.1. 


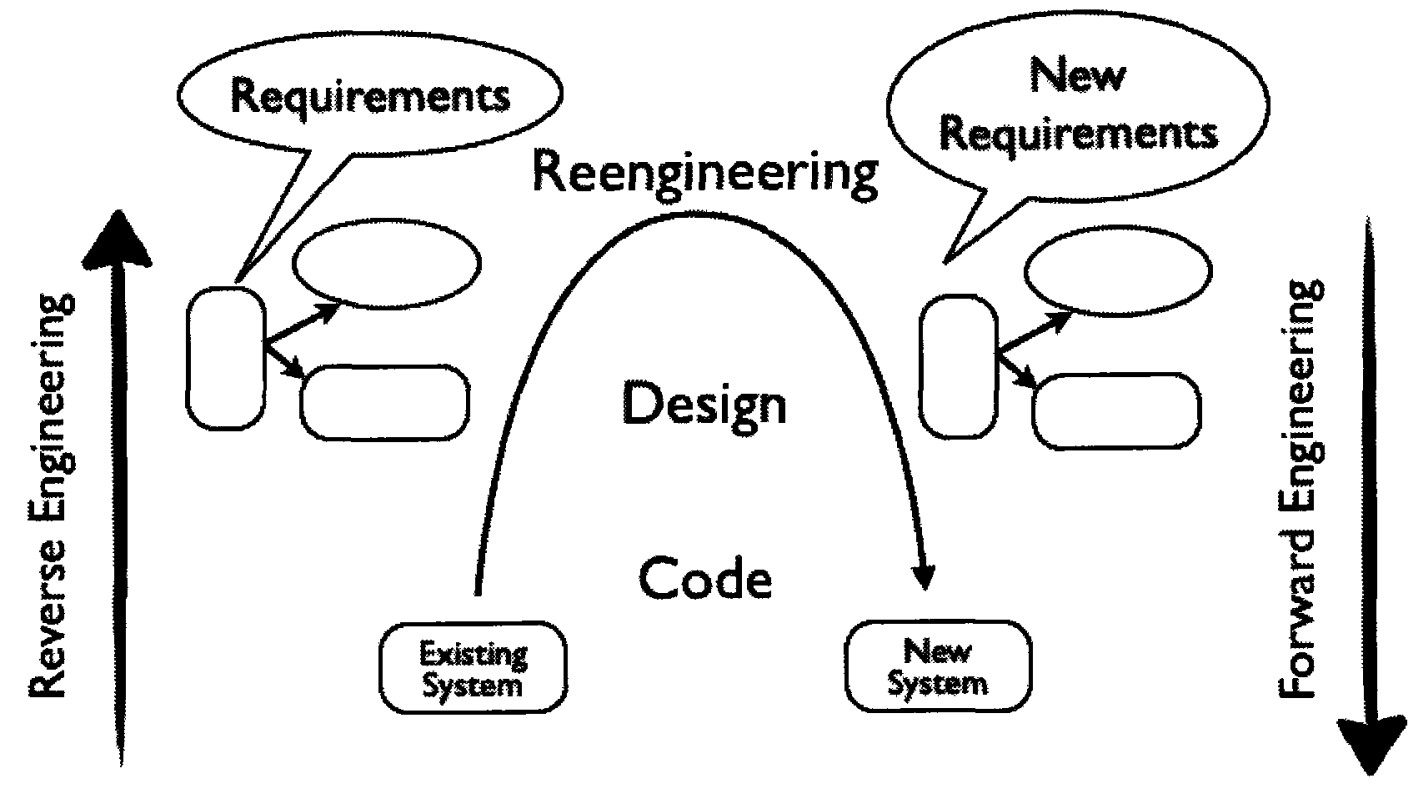

Figure 2.1 Forward, Reverse, and Reengineering [Dem03]

As the analysis goes backwards in the development cycle, the software system itself is not modified; merely its different modules are identified in the software architecture and their relationship with the specification and requirements. A higher level of abstraction representing the software system is being produced during this phase [Chi90]. The steps that are involved in reverse engineering are as follows in [Byr91] [Aik98]:

- Collecting information

- Examining information sources

- Extracting the structure

- Recording functionality

- Creating control-flow diagram 
- Reviewing recovered design

\section{Collecting Information}

Firstly, the overall information about the program and the software system needs to be collected as to understand which problem domain this software focuses on. This information can be program source code, design document, specification of APIs, overview of simulation of the system architecture, etc. In case of reverse engineering on a black-box software system, one can obtain only the specification of APIs, thus a thorough testing of the APIs will be necessary to perform as to know how far this software can go. Domain knowledge in related areas can be helpful, too, as they can be assets to assist in understanding the problem domain of the legacy software system.

\section{Examining Information Source}

Second, it is necessary to get familiar with the software, the architecture of the system and how it works. This procedure allows us to dissect the program of the software system, and match up the information obtained with the development environment.

\section{Extracting the Structures}

This step is to map out the different modules, layer architecture, and subsystems of the software. For better visual analysis, one can use the UML diagram to draw class diagrams, or a sequence diagram to visualize different components the software [Cla99]. Extracting the calling hierarchy, which will reveal all the modules/classes that are involved in such method invocation, can reproduce a sequence diagram. The sequence 
diagram can aid in understanding the control flows of APIs and what are the software's responses to external stimulus. A class diagram is also helpful in documenting the methods and attributes of classes in different modules, and it also reveals which data/information is being exchanged by observing the association between classes [UML97a][ UML97b][OMG07].

\section{Record Functionality}

If the specification of the software is provided, one can easily find out what the system can do in terms of its functionalities. Otherwise, one will have to reverse engineer to find out the function aspects of the software and jot down its functional requirements accordingly. The non-functional requirement of the software will be harder to record, because this depends on how obvious the system's behaviors are.

\section{Creating Control-flow Diagram}

With the structure extracted from the class diagram, one can identify the boundary/interface classes, entity classes and control classes. This task uncovers the actions to which the system can respond with both the internal stimulus (invoked by the system itself) and external stimulus (user input). The sequence of operations taken can be overlaid in a sequence diagram, clearly presenting a data/control flow of a specific stimulus, the functional aspect. This allows us to visually perceive both the data and control flow on the same diagram [Byr91][Chi90]. Thus, the number of sequence diagrams obviously depends on how complex the system is and how much input it handles. 


\section{Reviewing Recovered Design}

This step is to document the information gathered, and verify that the recovered design of the structure does describe the software system; that no functionality of the software has been overlooked and no unrelated information has been recorded. The idea is to document the recovered software architecture, not the software. Obviously, it is impossible to recover the design completely due to the following factors:

- Lack of knowledge in problem and application domain of the software

- The significance and meaning of the data held by different modules could not be documented, thus they can only be guessed at.

- Pre and post condition of operation could be unknown, meaning the range of possible input arguments is not clear.

To summarize software reverse engineering, this is a study/technique that decomposes the legacy software; each piece has commonality and structure as to avoid code redundancy and improve abstraction. It may further identify how the code can be further modularized to enhance code reusability during re-engineering [Rug93] [Byr91].

\subsection{Existing Concurrency Software Architecture Patterns}

The concurrency architecture patterns demonstrate a systematic and disciplined approach to tackle the problems in distributed and concurrent programming. They also provide a communication platform with robust, reliable and reusable solutions to various programming challenges. Design patterns are about design and interactions of objects, as well as providing a communication platform concerning elegant, reusable solutions to 
commonly encountered programming challenges [Sch00][Dat10]. The concurrency patterns that were used are documented in [Sch00]. These are dynamic thread creation (DTC), Leader/Followers (LFs), producer-consumer, and Half-Sync/Half-Async (HS/HA). Among the examples of concurrency design patterns that this thesis adopted, only the Dynamic-Thread-Creation (DTC) is a creational pattern, where the rest are all behavioral patterns. The reasons why these design patterns for concurrency architecture can help to improve an application's overall performance are as follows [Sch00]:

1. If a request is a mixture of CPU- and $\mathrm{I} / \mathrm{O}$-bound operations, the $\mathrm{CPU}$ cycle will be wasted when the task is blocked while the CPU is waiting for the $\mathrm{I} / \mathrm{O}$ operation to complete. In contrast, under the multi-processing or multi-threaded environment, the operating system can schedule the blocking process or thread to wait in the queue, allowing other processes or threads to perform their CPU-bound operations concurrently. The blocking threads will eventual join the wait queue when they finish their I/O-bound operations, and wait for the OS scheduler to dispatch the next thread to the CPU. As a result, the performance and CPU utilization is improved, and it will be more obvious, under a multi-processor hardware environment, where threads/processes can run concurrently.

2. The OS ensures that concurrency enhances the fairness and availability of CPU resources among threads through the scheduling policy. Thus the short running tasks will not get blocked too long because there is a long running task before it [Lea99]. 
3. Concurrency can improve the responsiveness of the software to external stimulus (user inputs). Usually the user of the software wants to receive responses quickly. The thread that handles the user input will become higher priority while other threads can run concurrently in the background to perform the necessary computations. This ensures that the long running task will not freeze the application screen [Shi00].

The following sub-sections describe each of the architecture patterns in more detail.

\subsubsection{Dynamic thread creation (DTC)}

DTC is a thread-per-request concurrency architecture, where a new thread is being created upon each arrival of a request. DTC is mostly seen in server application where a multi-threaded environment is required to ensure responsiveness. Thus, DTC will spawn a thread for each arrived request as shown in Figure 2.2. 


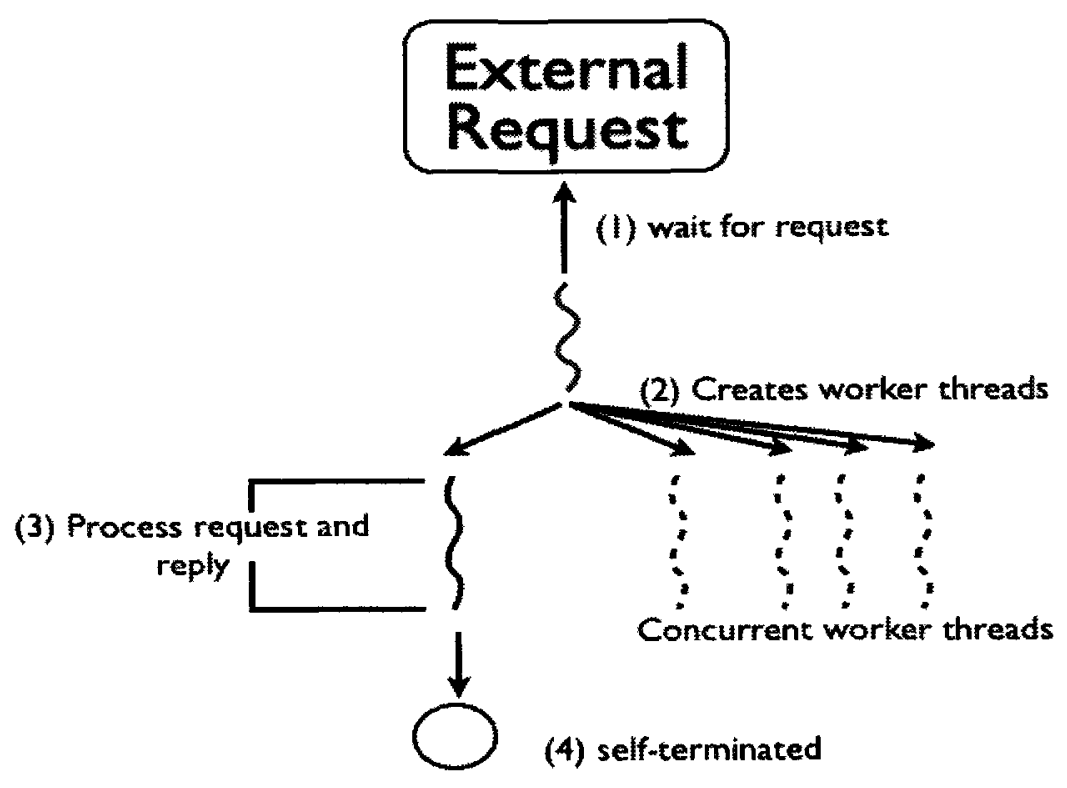

Figure 2.2 Dynamic thread creation [Zha10a]

DTC is fairly easy to program because programmers can simply spawn a thread and let the operating system or thread library handle the thread scheduling, creation and destruction details. DTC is usually used when the task being processed does not require intercommunication with other threads. Thus, the nature of each task is independent. However, there are two downsides of this approach, which are the overhead of dynamic thread creation and the number of threads created that the operating system can handle [Har05].

As stated, the operating system allocates resources to create a thread for each request, and there is an overhead associated with this thread creation. Thus, DTC is used when the amount of execution time taken to process the request is large compared to the 
time taken to create a thread. In this case, the ratio of thread creation to execution time of request is low.

If the arrival rate of requests is high and the execution time of requests is also long, then the number of threads being created in the system will be large. Once the DTC creates too many active threads in the system, the active threads will generate a huge amount of thread context switching activities [Zab98], which in turn will deteriorate the performance and responsiveness of the system [Har05].

\subsubsection{Leader/Followers (LFs)}

The concurrency architecture of LFs can help to minimize the concurrency-related overhead and prevent race conditions. Using LFs, there is only one leader thread in the system at all times, and its sole purpose is to wait for a request. Once the leader receives the request, it promotes one of the follower threads in the thread pool to become the leader, and it becomes a worker thread and proceeds on processing the request. When the thread finishes processing the request, it becomes a follower thread and returns to the thread pool, waiting its turn to become the leader thread again. Thus the active threads are those worker threads running concurrently. The leader thread can have higher priority than the worker(s) and follower(s) thread in order to intercept the incoming request as shown in Figure 2.3 [Sch00][Zha10a]. 


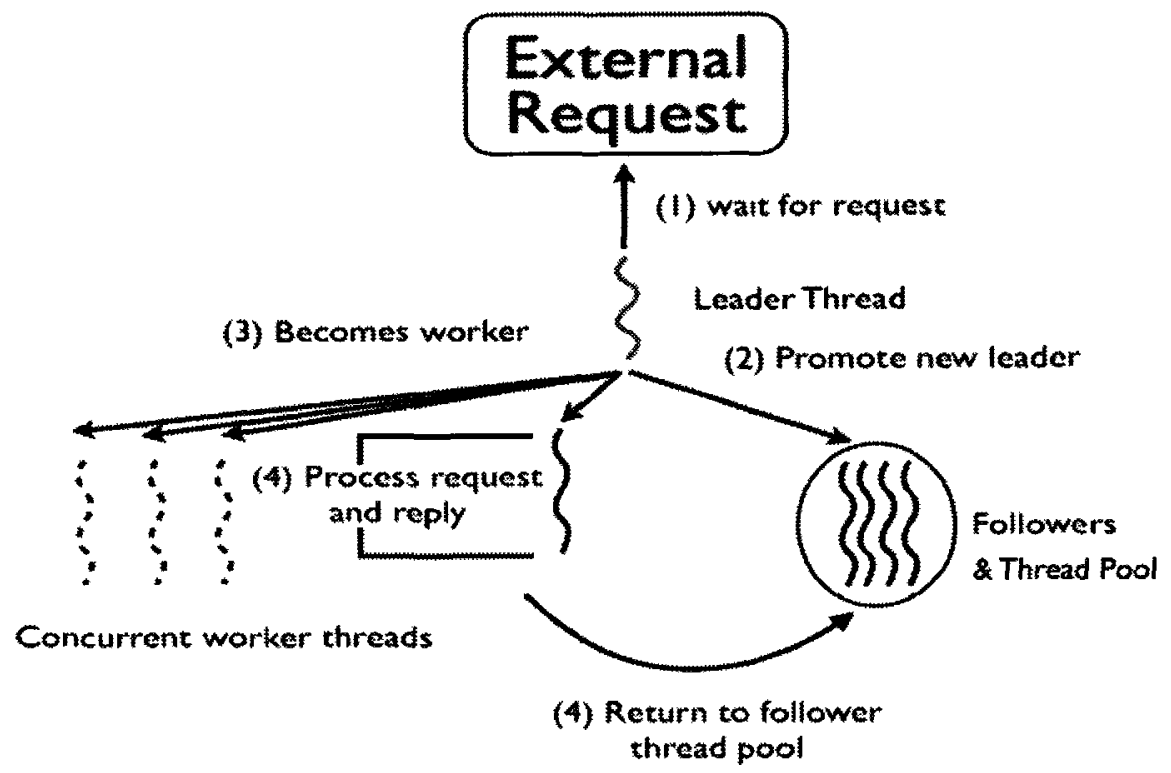

Figure 2.3 Leader/Followers concurrency pattern [Zha10a]

The critical/contention section of LFs is the thread pool as presented in Figure 2.3. Both the leader and workers have reference to the thread pool when they want to promote a follower thread to become the leader or return to the thread pool when worker threads terminate processing the request.

The advantages of using LFs are performance enhancements under an event driven environment and prevention of race conditions because there is only one leader thread listening to the event. In contrast, the disadvantages of LFs are a lack of flexibility, implementation complexity and the network $\mathrm{I} / \mathrm{O}$ bottleneck. The implementation complexity is due to the fact that the state of the threads is frequently changing concurrently. If the tasks consist of different execution time of CPU- and I/O-bound, it is difficult to tell what the order of execution is while the system needs to maintain 
operation atomicity. Moreover, LFs does not have a queue, thus it is more complex if the programmer wants to reorder the requests with different priorities [Sch00].

\subsubsection{Producer-Consumer (PC)}

The PC problem is also commonly known as bounded-buffer problem as illustrated in Figure 2.4. In the simple PC concurrency architecture, the producer can be a thread/process, which runs concurrently with the consumer thread/process. The producer runs in a loop, constantly generating data and putting them into the bounded buffer. The consumer, on the other hand, consumes the data by extracting the data from the bounded buffer. It may take some time for the producer/consumer to generate/consume the data, thus the accessing time of the bounded buffer is unpredictable.

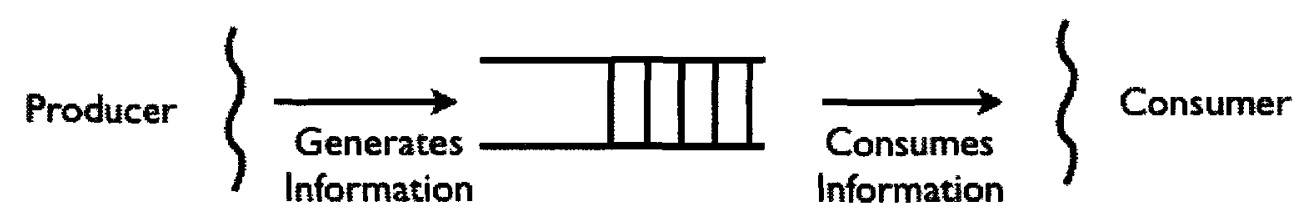

Figure 2.4 Producer-Consumer problem [Oak04]

Since the order of accessing the bounded buffer (critical section) is unpredictable, the data being queued up in the buffer must be conducted in an orderly fashion with a synchronization mechanism, e.g., mutual-exclusion lock or semaphore. This synchronization mechanism will ensure that writing and reading from the buffer is always consistent. The advantages of the PC problem are [Oak04]:

1. Decoupling of the work between the producer and the consumer, so they can operate concurrently at different rates 
2. Management of the bounded buffer size is flexible according to the system's needs

3. Separation of concerns: both the producer and the consumer can be replaced or modified without affecting each other.

\subsubsection{Half-Sync/Half-Async (HS/HA)}

The HS/HA concurrent architecture, presented in Figure 2.5, is a modified Producer-Consumer problem, where HS/HA has only one producer (the asynchronous layer) and multiple consumers (the synchronous layer). The asynchronous layer mainly deals with listening and receiving the requests, and then saves the requests in a message queue: the queuing layer. Thus the asynchronous layer usually possesses higher priority as to capture requests to avoid any packets loss. The synchronous layer's main purpose is to decode the requests being stored in the queuing layer and perform the necessary $\mathrm{I} / \mathrm{O}$ and/or computational operations. HS/HA has the same advantages as the PC pattern in decoupling the asynchronous and synchronous services in a multi-threaded and concurrent system [Sch96]. 


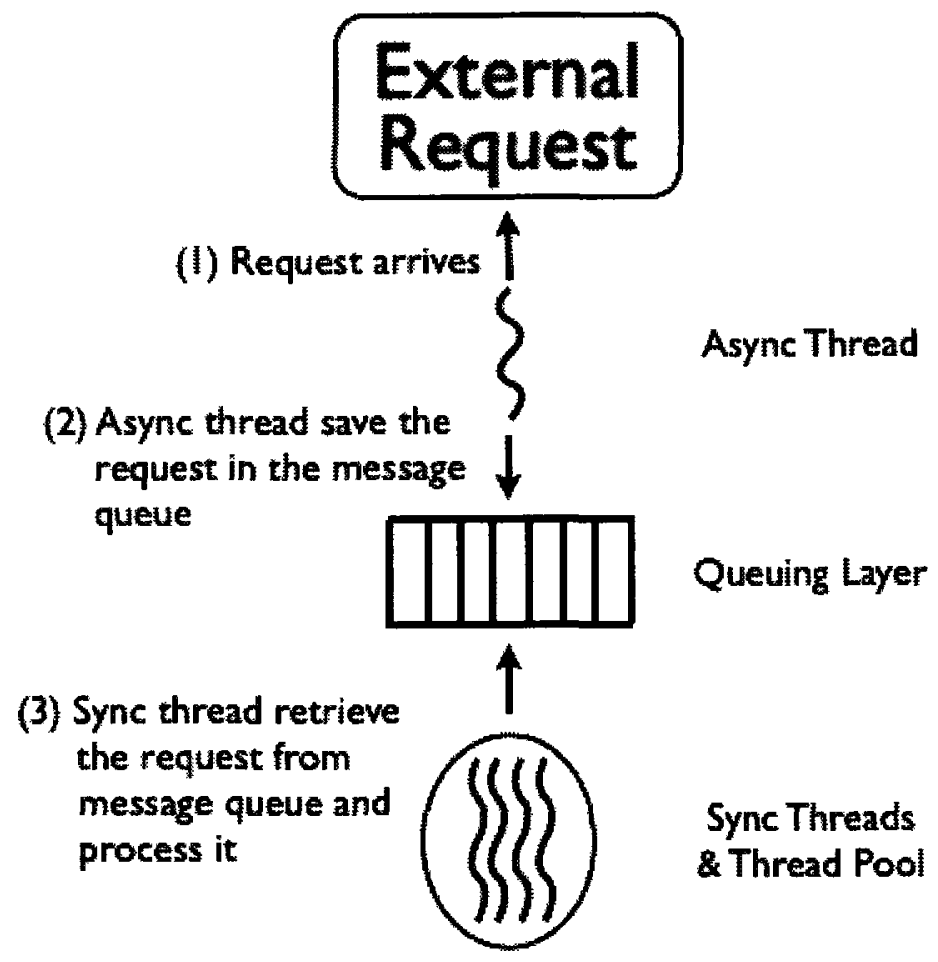

Figure 2.5 Half-Sync/Half-Async Architecture [Sch96][Zha10a]

The major functionality of the queuing layer is to decouple the asynchronous and synchronous threads, and buffer the requests into the message queue. In the HS/HA architecture, each layer has low coupling to each other and can perform operations concurrently and independently. However, the queuing layer is where the critical section resides; i.e., no request should be put into the message queue when it is full, and no synchronous threads should read the message queue whenever it is empty. Another limitation of HS/HA is the context switching overhead of threads and the synchronization of the critical section (message queue) when requests are transferred from one layer to the other [Sch00][Sch96]. 


\subsection{Aspect Orientation}

Aspect-Oriented Programming (AOP) is a further extension of the existing Object-Oriented $(\mathrm{OO})$ programming. AOP enhances the capabilities of $O O$ in separation of concerns, scalability of services and maintainability. AOP aims at the management of crosscutting concerns. Concerns are the system requirements or features. These concerns are the same nature, and they can be populated in several modules/classes of the system. These concerns crosscut (crosscutting concerns) modules/classes and create difficulties in maintenance and degrade modularity of the system. This AOP paradigm enables the software and system developers to extract the crosscutting concerns embedded in different parts of the system and centralize each concern into a module, the so called aspect, to achieve cleaner code. Thus, aspect is the modularization of concerns implemented across various modules/classes.

\subsubsection{Aspect-Oriented Programming}

The difference between the traditional OOP paradigm and AOP paradigm is illustrated in Figure 2.6. The traditional OOP approach abstracts similar functionalities or characteristics of classes together and creates a high level abstraction of modules or packages in a layered architectural approach [Bla10]. However, this approach can leave a certain level of code scattering and code tangling. Thus, whenever the concerns need to be changed using OOP, many of the classes in different modules can be potentially modified. This action can compromise the integrity of the system and the effect can ripple across various parts of the system, which causes difficulties in maintaining the 
structure of the whole software system. On the contrary, AOP can maintain the structure of the original software system, change existing concerns, add new concerns, or even replace aspects without affecting other parts of the system. In general, an AOP-based approach should be more efficient than OOP in terms of modularity.

OOP Paradigm

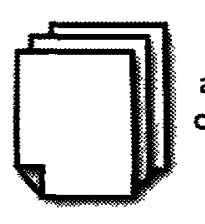

adding crosscutting

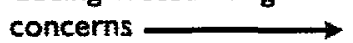

AOP Paradigm

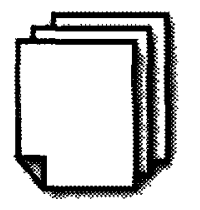

adding crosscutting concerns
Objects

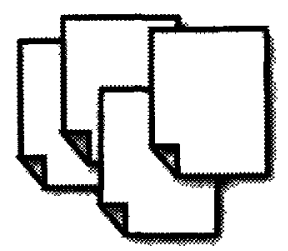

Objects



changing

concerns

Aspect

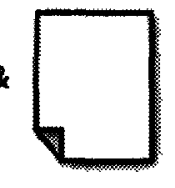

Objects

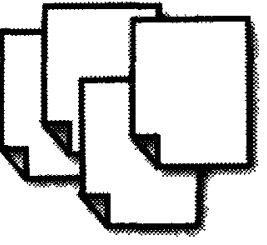

concerns



Figure 2.6 Management of crosscutting concerns with OOP and AOP [Sub05]

The two issues in the traditional OOP, which are mostly mentioned in AOP are code scattering and code tangling. They are considered symptoms of non-modularization [Lad03] and these symptoms are what AOP is trying to improve. Brief descriptions of code scattering and code tangling are as follows:

- Code tangling is when code with mixing and/or multiple concerns are embedded within the same module/class object.

- Code scattering is when the same issue or repeated code is implemented in multiple module/class objects. 


\subsubsection{Aspect-Oriented Concerns}

A desirable design of modularity of the core concern is to encapsulate the central functionality of a module/class object, so the module/class object focuses on its major task-related concern only. A crosscutting concern is a functionality of system-level requirements, which appear in multiple modules/classes of the software, and such crosscutting concerns are what AOP will capture and encapsulate into an aspect. Some common concerns, which cut across modules/classes, are shown in Table 2.1.

\begin{tabular}{|c|c|}
\hline Concerns & Examples \\
\hline Reliability & Logging, Tracing \\
\hline Security & Authentication, Authorization \\
\hline Correctness & Invariant maintenance \\
\hline Performance & Buffering, Pooling, Caching \\
\hline Functionality & Billing, Taxes \\
\hline
\end{tabular}

Table 2.1 Common crosscutting concerns [Lea10]

Figure 2.7 illustrates OOP based software with concerns scattered around at all different modules/classes of the software. Each color represents a type of crosscutting concern as demonstrated in Table 2.1. A code tangling is when a module/classes object performs more than its core concern, as shown by multiple colors (concerns) within the same module/class object. For instance, in Figure 2.7, each module/class has the issue code tangling, except the one in the middle, has more than one color. Code scattering on 
the other hand, is when the same color (concern) is observed in different modules. In Figure 2.7, red, green, blue, and purple appear in multiple module/class.

OOP Paradigm

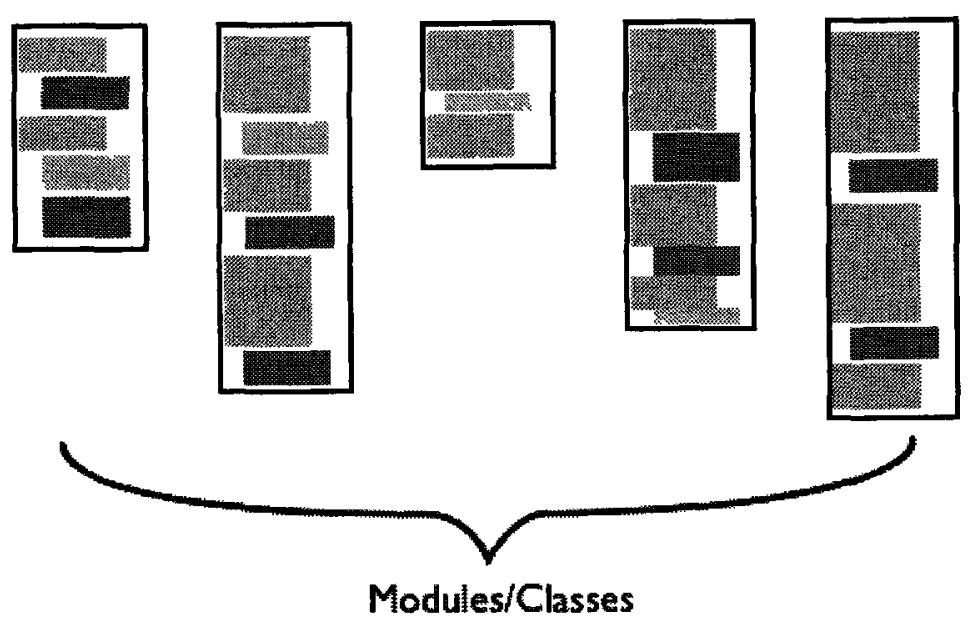

Figure 2.7 Crosscutting concerns implemented in an application [Lea10]

\subsubsection{Reusable Aspect-Oriented Implementation}

As the crosscutting concerns are extracted from the modules/classes and encapsulated into aspects, software developers can extend, implement and subclass aspect modules like the regular OO modules. This means that interface and inheritance also exist in aspect modules. The aspect modules then become highly reusable. There are mainly two approaches of using these reusable AOP [Lea10]:

1. Identify and separate concerns, which are embedded across the functional components.

2. Integrate the new feature into the existing system without affecting the original functionality. Such newly added features are results of unforeseen functional 
and non-functional behaviors during the design phase of the software engineering development.

Both of these approaches can help produce a cleaner design and substantial code savings. Figure 2.8 illustrates an example of concerns being extracted from different modules in Figure 2.7. Each of these extracted concerns is later encapsulated into an aspect represented by a color. Each aspect can be functional or non-functional behavior of the applications listed in Table 2.1. AOP enables programmers to exploit most of the features of OO paradigm on AOP paradigm, thus inheritance, abstraction, and interface are all implementable in aspect modules [Ape08a][Cun06].

\section{AOP Paradigm}

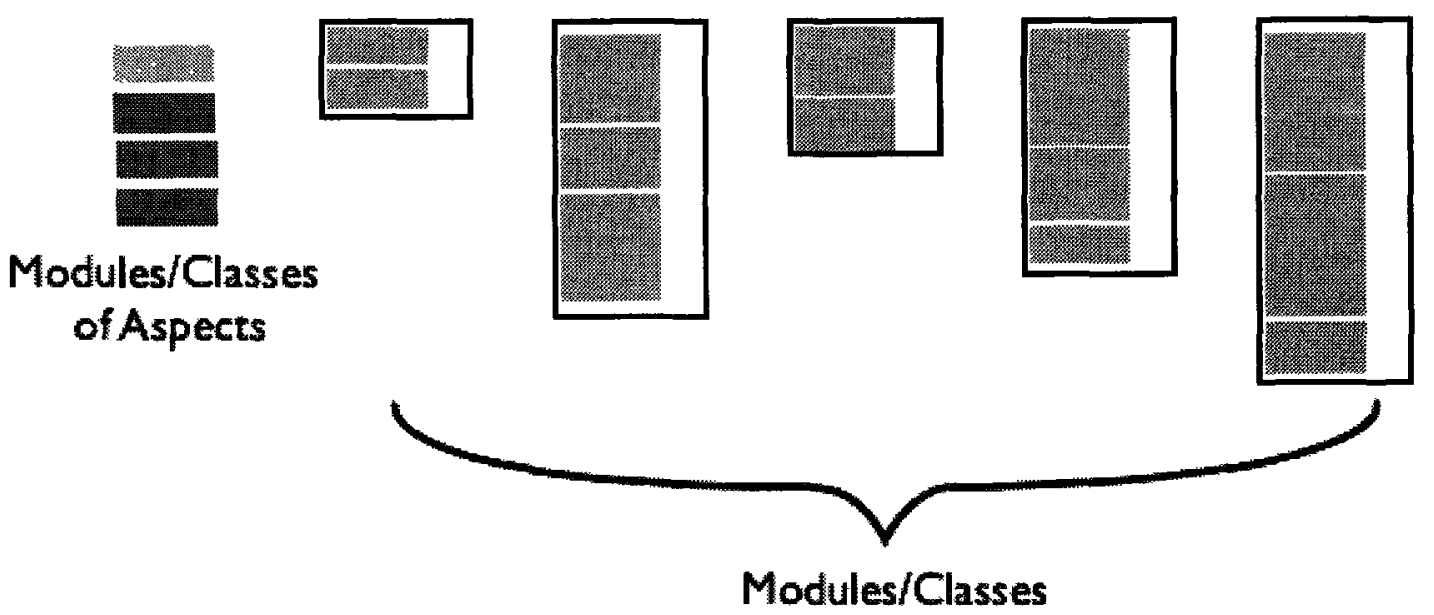

Figure 2.8 AOP based approach to encapsulate crosscutting concerns into Aspects [Lea10]

Aspect can be further categorized into two types: development aspects and production aspects. Development aspects are those aspects used during the development 
process of the software. Some of the development aspects can further become the functional aspects or non-functional aspects, and will remain in the final product. The non-functional aspects of testing and debugging purposes will be removed from the product. Thus, those aspects that remain are addressed as production aspects. These modularized aspects help to improve design and reusability by enhancing the principle of separation of concerns. The resulting program provides cleaner distribution of responsibilities with higher modularization for system evolution, and produces a software system that is easier to maintain and understand [Sor07][Sub05].

\subsection{Summary and Scope of the Thesis}

The common thing between LFs and HS/HA is the thread pool. The thread pool is the place where threads are pre-allocated. DTC on the other hand spawns a thread as per request on the fly when a request has been captured. The major difference among these concurrency architecture is that the thread pool has threads pre-allocated after the initialization of the system, while threads spawned by DTC are self-terminated after they are finished with the request. The pros and cons of these three concurrency architecture are stated in [Lun06], [Sch00] and [Zha10a].

The concurrency architectures mentioned above are very commonly used today, and they have been proved for their robustness and efficiency. However, as the system gets bigger and larger, the architecture of the software becomes more complex over time. System requirements and features can be populated within multiple modules/classes. 
Such problems are called code scattering and tangling. These problems can be solved by using AOP.

AOP paradigm originates from the same OOP approach but focuses mainly on the extraction and modularization of concerns. These concerns complete the system requirements and features of the software, but are all scattered around in different modules/classes. AOP helps to centralize concerns of the same nature in one place, namely aspect. The rest of this thesis is dedicated to two common concurrently distributed structures, HS/HA and LFs, with AOP paradigm added. The performance impact of how AOP may affect the transition between base code and aspect code depend on the different parameters that should be exploited. 


\section{Chapter 3 Extracting Aspects and Identifying Crosscutting}

\section{Concerns from the Concurrent Architectures}

\subsection{Introduction}

A common concern for concurrent programming is the critical section of the software. This critical section must be synchronized to avoid race conditions and prevent data inconsistency. Other concerns which need to be synchronized may be the management of scheduling policy due to the threads created, and the assignment of tasks that can run in a separate thread. These concerns contribute to the negative phenomena of code scattering and code tangling in the software [Ape08b]. Designing such concurrent applications is difficult because it is impossible to know the order of threads being activated. In addition, the scheduling of threads depends on the OS and Java virtual machine.

Even though Java [Lea99][Oak04][Goe06] has evolved and has been improved for the development of concurrent applications with some specialized libraries in Java 1.5 , Java was not explicitly designed to address the crosscutting phenomena. It is still possible for programmers to build software that has scattering and tangling issues using traditional Java.

To help programmers deal with the complexity of concurrency and avoid the scattering and tangling problems, this chapter presents the application of AOD and AOP for two well-known concurrency patterns, HS/HA and LFs. 


\subsection{Aspect-Oriented Language}

Aspect-Oriented Programming is a well known paradigm that provides the programmer with the ability to separate crosscutting concerns in a modular fashion and still use the traditional OOP paradigm [Kic97]. AOP further supports new behaviors of switching AOP's code (advices) at some very specified points (join points) of the program, while still maintaining the system's modularity and architecture. There are many providers and open source tools available that support AOP, such as AspectC++, JBoss AOP, Aspect\#, Jasco, Spring AOP and AspectJ [Ker05][Wik10b]. The differences between these AOP languages can be found in [Ker05]. There are three major differences of how AOP is implemented: using code style, annotation, and xml [Ker05]. There are pros and cons of each one. There is no exact answer as to which AOP language to choose. It depends on the application domain and feasibility of the software developer.

With all different AOP languages supported in the market, AspectJ is chosen as the platform for this research because it is used most in the industry and academic research. AspectJ is also an open source project in Eclipse [Asp10], which is also one of the most commonly, used Java development platforms. Furthermore, AspectJ can be imported into other Java platforms, like NetBeans, JBuilder, Emac and JDEE.

\subsubsection{Semantics of AspectJ}

The terminology of AOP is not intuitive, but fortunately most of the AOP languages use the same terms in naming the semantics [Gra03][Spi10] as shown in Table 3.1 . 


\begin{tabular}{|l|l|}
\hline Terminology & \multicolumn{1}{|c|}{ Explanation } \\
\hline Join Point & $\begin{array}{l}\text { A specific point within the execution of the program. It can be } \\
\text { method execution, method call, constructor execution, object } \\
\text { instantiation and exception handles. }\end{array}$ \\
\hline Pointcut & $\begin{array}{l}\text { It is a language construct, which defines the specific group of join } \\
\text { points for inclusion of a new behavior, namely advice. Thus, these } \\
\text { particular join points which match up with the pointcut definition } \\
\text { will be added with new functionalities, i.e., new code will be weaved } \\
\text { into the original program where the join points are. Pointcut } \\
\text { description (PCD) allows Boolean operations and wildcard pick join } \\
\text { points flexibly. }\end{array}$ \\
\hline Advice & $\begin{array}{l}\text { Actions and behaviors taken at the join points specified by the } \\
\text { Pointcut Description (PCD). }\end{array}$ \\
\hline
\end{tabular}

Table 3.1 Terminology of AspectJ [Gra03][Spi10][Cha03]

\subsubsection{Different Types of Advice}

An advice is a behavioral action included at the join point where it matches up. There are five different types of advice in AspectJ [Gra03][Spi10] as shown in Table 3.2. 


\begin{tabular}{|c|c|}
\hline Advice & Explanation \\
\hline Before & $\begin{array}{l}\text { An advice that runs before the execution of a join point. Setting up or } \\
\text { checking up the pre-conditions can be done within this advice. }\end{array}$ \\
\hline Around & $\begin{array}{l}\text { An advice that runs before and after a join point. A custom behavior } \\
\text { can perform before and after the joint point, and it can also choose to } \\
\text { proceed to the join point or completely bypass the advised join point } \\
\text { by returning its own processed return value. }\end{array}$ \\
\hline After returning & $\begin{array}{l}\text { An advice which gets executed when the execution of a join point } \\
\text { complete normally without throwing any exception. }\end{array}$ \\
\hline After throwing & $\begin{array}{l}\text { An advice that gets executed when an execution of a join point exits } \\
\text { by throwing an exception. }\end{array}$ \\
\hline After & $\begin{array}{l}\text { An advice that gets executed no matter what kind condition does the } \\
\text { join point exit. }\end{array}$ \\
\hline
\end{tabular}

Table 3.2 Different types of advice [Gra03][Spi10][Mil05]

\subsection{Extraction of Aspects from LFs Concurrent Architecture}

The concurrency architecture of LFs has one leader and multiple follower threads. Essentially, these threads are of the same type, LFsThread. The state of threads differs, and such states determine whether they are an active or non-active thread in the system. The structure of the existing LFs concurrency architecture was captured and converted into a UML class diagram as shown in Figure 3.1. There are mainly five different classes in this system. In this structure, multiple LFsThread threads share the same instance of 
LFsThreadPool pool. The thread pool itself ensures the correctness of coordination among the threads [Zhal0a].

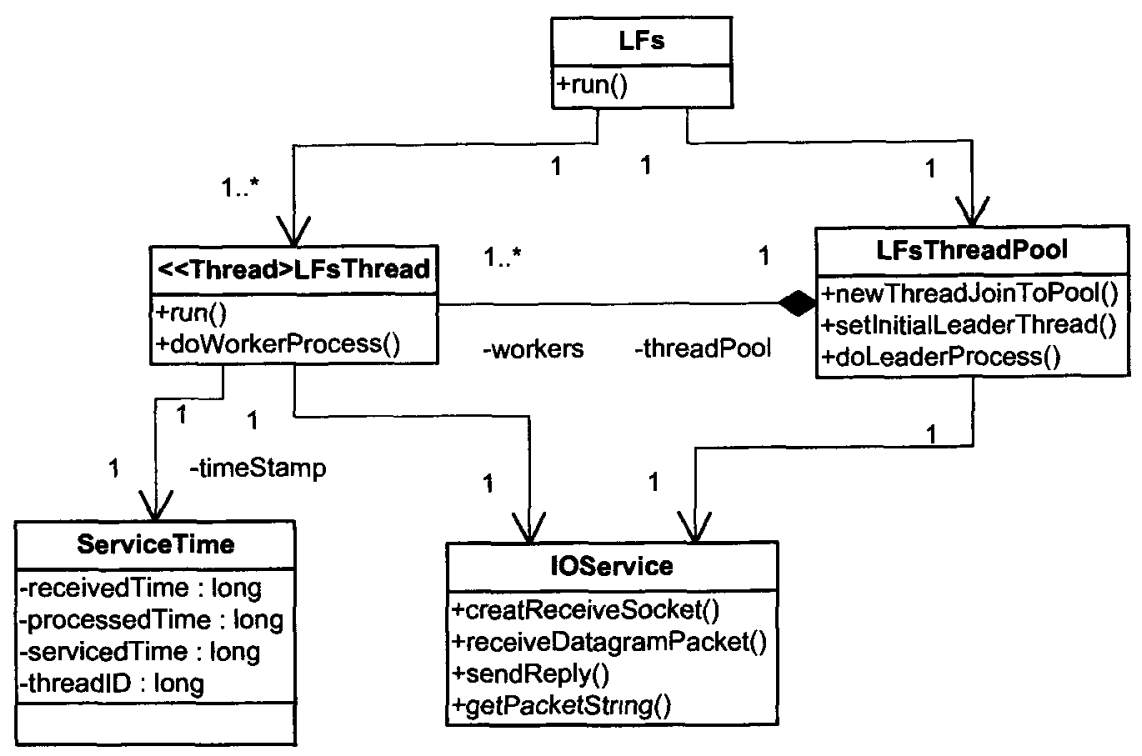

Figure 3.1 Class diagram of LFs [Zha10a]

LFs is the first class being instantiated. Its main function is to create the thread pool and generate multiple threads, and then give the directives to the threads created to join them to the thread pool. $L F s$ itself is a thread as well. Once the thread completes its task, it terminates and exits.

LFsThreadPool is where the contention of the leadership resides. The threads in the pool all share a common synchronized lock and perform the same action for getting the leadership and promote a new leader before resigning as a leader. The threads have three states: leader, follower and worker. The role of the leader thread is to listen to external requests as presented in Figure 2.2. Once the leader thread receives a packet of 
request, it moves on promoting one of the follower threads to become the leader and resigns the leadership.

LFsThread is a general class definition of instantiated threads. All the threads, leader, follower and worker threads, are instances of this class. Unlike the LFsThreadPool instance, LFsThread is a thread. The follower threads try to obtain the access right of the thread pool in order to receive the external request. When a thread obtains the access right, its thread-state changes to leader. Once the request is captured, the leader thread will give up the access right of the thread pool and promote one of the follower threads to become a leader thread. The resigned leader thread becomes a worker thread and carries on processing the request. At all times, there is only one leader thread possessing the lock of the thread pool in order to ensure the correct coordination of all threads. At the end, the worker thread terminates the task and returns to the thread pool to become a follower thread, waiting for its turn to become the leader again.

IOService is a class composed of a few methods for $\mathrm{I} / \mathrm{O}$ operations, for example, creating sockets, sending and receiving data packets and extracting message from the encapsulated packet.

ServiceTime is a class for recording relevant information of data or request processing. It consists of the time slot when the request is received, when a request is sent, the amount of time taken to process the request, and the thread's ID which indicates which thread is responsible for such request. 


\subsubsection{Identification of Crosscutting Concerns for LFs}

Since the LFs concurrent architecture is a concurrency architecture, the most obvious crosscutting concern is the concurrency issue dealing with synchronizing the lock on the thread pool. Figure 3.2 illustrates the class diagram with the aspect that handles the concurrency feature. The concerns of how the leader is chosen and their respective thread priority scheduling in the original base design was implemented in two classes, which are LFsThread and LFsThreadPool illustrated in [Zha09]. These concerns which spread across in LFsThread and LFsThreadPool are identified and abstracted out into a new aspect class: SyncPriorityAspect. These concerns can be identified by the use of concurrency control code like synchronized, wait, notifyAll, and Thread.setPriority. 


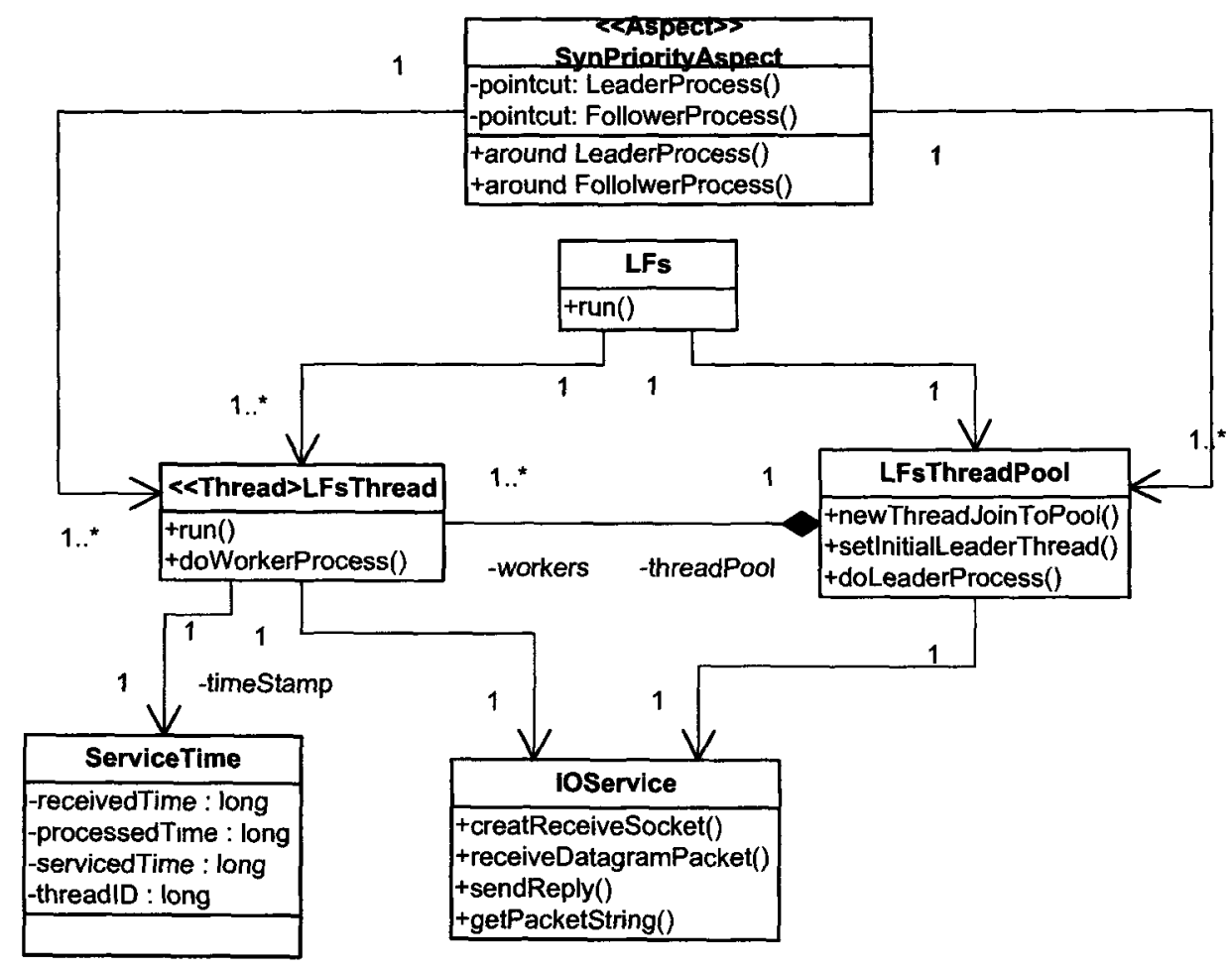

Figure 3.2 Modified Class diagram of LFs with aspect module

SyncPriorityAspect is a class of stereotyped aspect. This class consists of two pointcuts and two advices. Each pointcut selects its specific join points according to the pointcut description (PCD). The around advice is weaved into the original program whenever the program counter reaches the join point. 




\section{Figure 3.3 AspectJ code of the aspect class}

The PCD can be seen from line $10 \sim 13$ in Figure 3.3. The rest of the code is the body of the advice, which uses the around advice to bypass the original code. However, using the special call proceed (o can return the execution flow of the program back to the original method invocation. This proceed() call can be invoked anywhere within the around advice. The advices here are dealing with the thread priority, which is giving the 
leader thread more precedence than the worker thread. Thus the leader thread can preempt all other running threads and become the only active thread in order to capture the incoming request from the socket. At line 19 of the code, the threadPool object is being synchronized, thus the advice has the lock of this object even when the program counter returns the control flow back to the original program with the proceed () call. The complete code of AspectJ and the class they advised are documented in Appendix A.

Figure 3.4 presents the sequence diagram for the new design based on AOD. There are four objects: LFsThread, LFsThreadPool, IOService and SynPriorityAspect. SyncPriorityAspect is an aspect object; the other three objects are obtained from the original baseline design. 


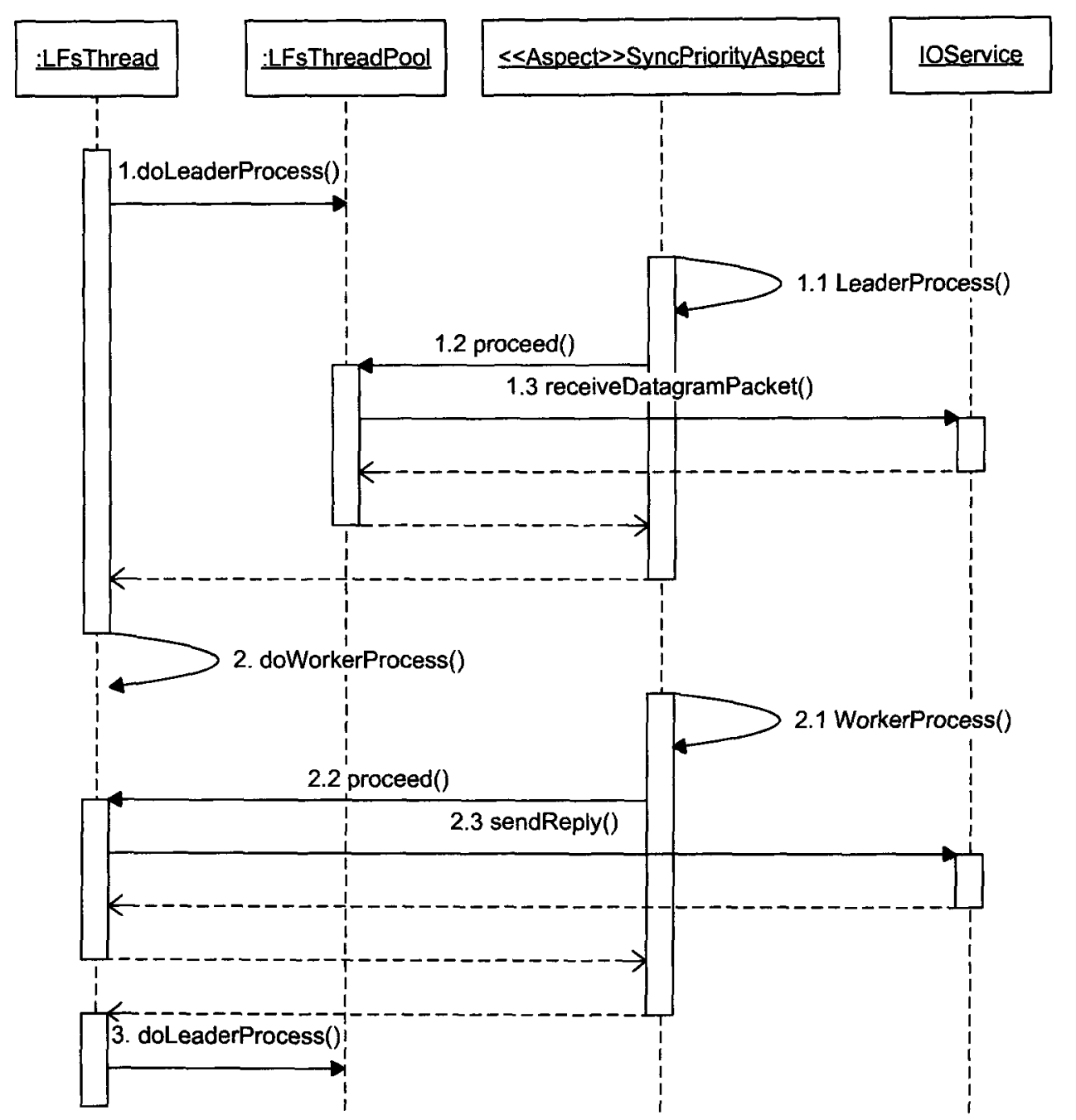

Figure 3.4 Sequence diagram of LFs with Aspect enabled

Aspect-Oriented programming has the ability to intervene the original base program as demonstrated in Figure 3.4. When doLeaderProcess () is called (message 1) upon the instance object of LFsThreadPool, it is noted that such an invocation is not active until message 1.2 called proceed() from the advice, which returns the control flow 
back to the base application. The instance object of $L F s T h r e a d P o o l$ has no idea of such intervention from the aspect. The same effect of doWorkerProcess() is illustrated at message 2. Notice that the message doWorkerProcess( is called within the same object, and it is also identified as a join point, thus advised by an advice. Therefore, the object of LFsThread is out of the scope when the advice intervened. It becomes active till the advice returns the control flow at message 2.2 called proceed(). A clearer illustration is shown in Figure 3.5. 


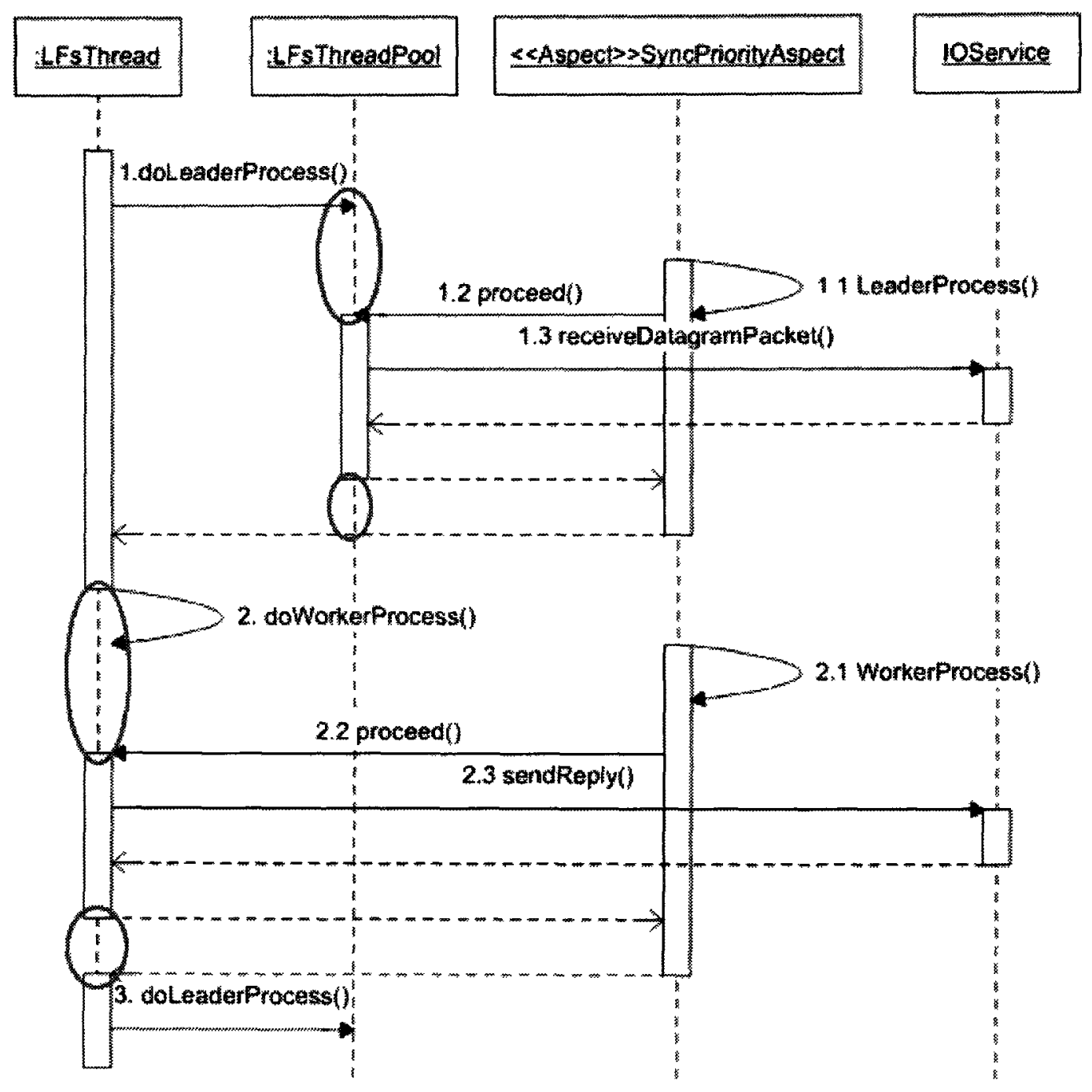

Figure 3.5 Sequence diagram of LFs indicating when the advised objects are inactive

The following steps explain the execution sequence presented in Figure 3.5:

1. An LFsThread object invokes doLeaderProcess () message in LFsThreadPool. In the original base design, the message will be activated immediately. However, with the doLeaderProcess $($ having been captured 
as a join point in the SyncPriorityAspect aspect, the control flow will be moved from LFsThread to SyncPriorityAspect.

2. The advice, LeaderProcess(), is executed when the control flow is obtained from the base program.

3. Within any part of the advice LeaderProcess(), proceed() can be invoked to return the control flow back to the original base program. The proceed() call will execute the original message, doLeaderProcess(), invocated from the base program.

4. Once doLeaderProcess() is done with its operation, the control flow will be moved to the aspect again. At this time, advice LeaderProcess() can ensure post-condition of the message doLeaderProcess 0 and perform additional control or logging information if needed.

5. SyncPriorityAspect will move the control flow back to LFsThread. LFsThread then carrying the calling message doWorkerProcess(), which is also captured as a join point by SyncPriorityAspect.

6. SyncPriorityAspect calls the message WorkerProcess() to update the priority of the thread, and ensures the pre-condition of doWorkerProcess(). Then SyncPriorityAspect moves the control flow back to doWorkerProcess() by invoking the message proceed().

7. When doWorkerProcess () is done, the control flow is returned to SyncPriorityAspect. 
8. SyncPriorityAspect ensures the post-condition of doWorkerProcess(), then moves the control flow back to the original program.

The red oval shape reveals the time length of how long the original base program is inactive due to the intervention of advices from SyncPriorityAspect.

\subsection{Extracting Functional Aspect from HS/HA}

The process of reverse engineering HS/HA is exactly the same as LFs', that is it undergoes the same reverse engineering process of LFs. The functional aspects that cut across multiple modules/classes in HS/HA are identified for AOD and AOP.

\subsubsection{Extracting HS/HA Concurrent Architecture}

The HS/HA concurrency architecture shown in Figure 2.5 of the existing code is converted into a UML class diagram as presented in Figure 3.6. There are mainly six different classes in this program. Unlike LFs, there is a queuing layer in between external events (asynchronous) and internal computation (synchronous). The worker threads in this case are the multiple synchronous (sync) threads, whose tasks are to de-queue the request form the message queue one at a time. Then the synchronous thread performs $1 / O$ and computational bound operations, and replies the result back to the original source of the request. The asynchronous (async) thread functions like the leader thread in LFs. However, the asynchronous thread does not resign from listening to the external event in HS/HA. 


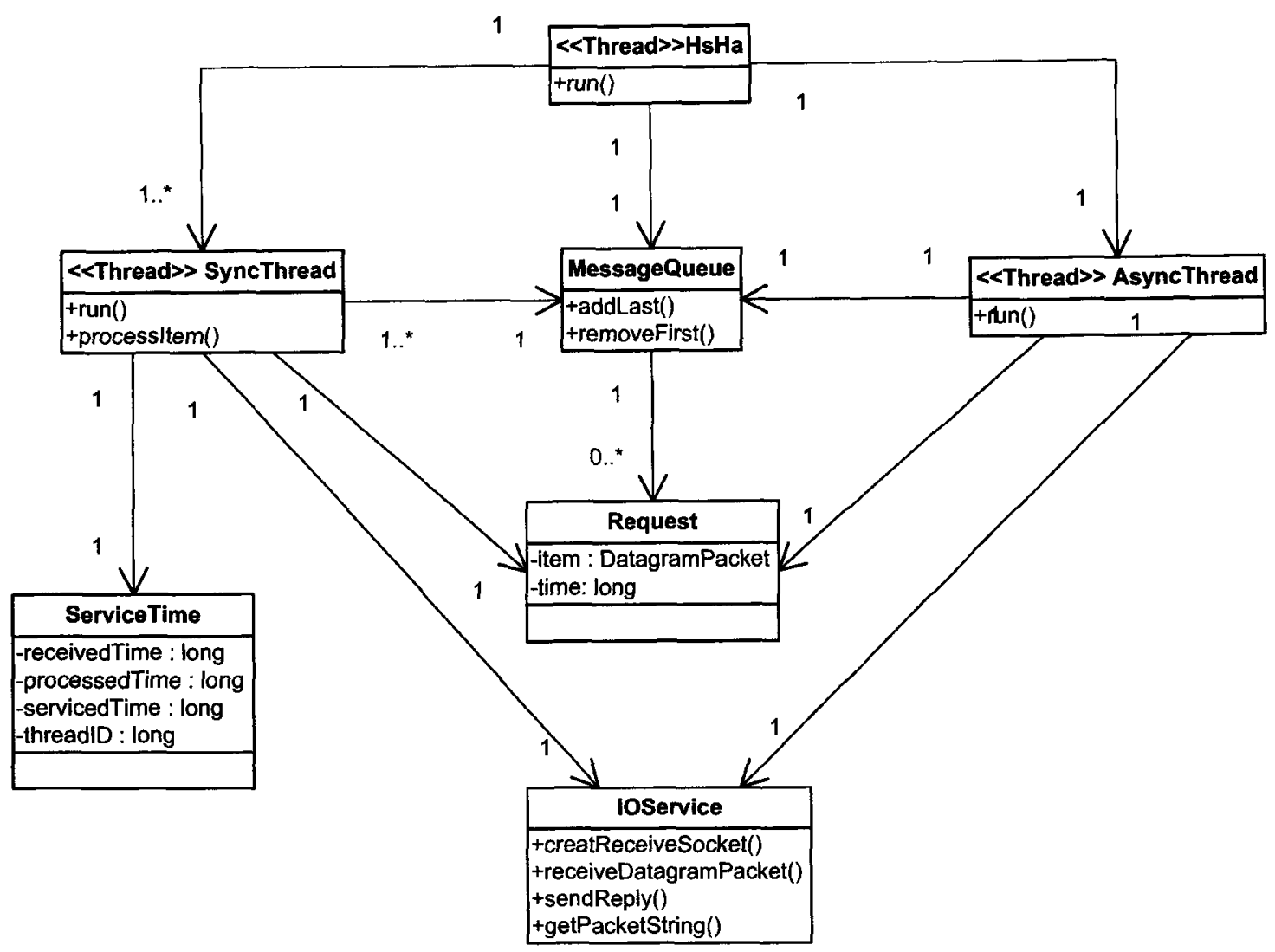

Figure 3.6 Class diagram of HS/HA

HsHa is the first class being instantiated. Its major functions are to create a async thread, a message queue and multiple sync threads. $\mathrm{HsHa}$ also has to link the references of the message queue to synchronous threads and asynchronous threads, and then release all the threads to active state.

AsyncThread is the asynchronous thread instance. There is only one thread of this type in HS/HA, and its major function is to stand by, listening to socket for incoming request. The asynchronous thread encapsulates the request as a message object and 
moves it to the message queue. The thread priority of an asynchronous thread cannot be lower than the synchronous threads [Zha08], otherwise the system will experience serious packet loss if the arrival rate of requests is much higher than the service rate [Sch00][Oak04]. However, an asynchronous thread can be either higher or equal to the synchronous threads' priority, so that an asynchronous thread can preempt or time-slice with all other threads.

SyncThread is the class definition of synchronous threads. There is at least one of this instance being instantiated in HS/HA. Their major functions are to run, de-queue the message from the message queue, verify what the request is, and perform the CPUand I/O-bound concurrently. All the synchronous thread objects have the same priority. The number of this type of thread is bound to a specific threshold. Overloading the system with too many threads not only consumes the hardware resources, but also degrades the overall performance due to the thread contention and context switching among threads.

MessageQueue is the class definition of the message queue instantiated in the queuing layer. It is the bounded buffer in the Producer-Consumer problem. Its function is to store the encapsulated request from the asynchronous layer and to de-queue the request in a first-in-first-out FIFO style when the synchronous threads come to retrieve it. Therefore, the queuing layer is dealing with a single writer (async thread) and multiple readers (sync threads) in this HS/HA concurrency architecture. The message queue has to be locked for single access (storing and retrieving) at all times to ensure data consistency. 
Request is the class definition consisting of two fields, which are item and time. item is the DatagramPacket sent from the client. It can be used to retrieve the address of the client afterwards by the synchronous thread when performing the $\mathrm{I} / \mathrm{O}$-bound operation. time is the timestamp taken at the very moment when the async thread captures the request from the socket.

IOService and ServiceTime are the same as those in LFs as shown in Figure 3.1. They are the common classes that both LFs and HS/Ha use in their concurrency architecture.

\subsubsection{Identifying of Crosscutting Concerns for HS/HA}

There is also a place where contention takes place in HS/HA concurrency architecture, which is at the queuing layer. Both the asynchronous thread and synchronous threads have to access the message queue in order to accomplish their works. Thus, some code scattering of synchronization aspects on the queue can be extracted as a concern of functional aspects and encapsulated into aspect module/class [Zha10b][Sub05][Zha08]. The functionality of extracted synchronization aspect is to ensure that, at most, one active thread is accessing the message queue at all times in this concurrent environment. Encapsulating this concern as an aspect module/class, the synchronizing of the message queue is done obliviously by the aspect module/class. Both the synchronous threads and asynchronous thread don't have to know the existence of the aspect module/class at all. 
The identification of code that deals with concerns of synchronization can be spotted by the use of the concurrency control code like synchronized, wait, and notifyAll.

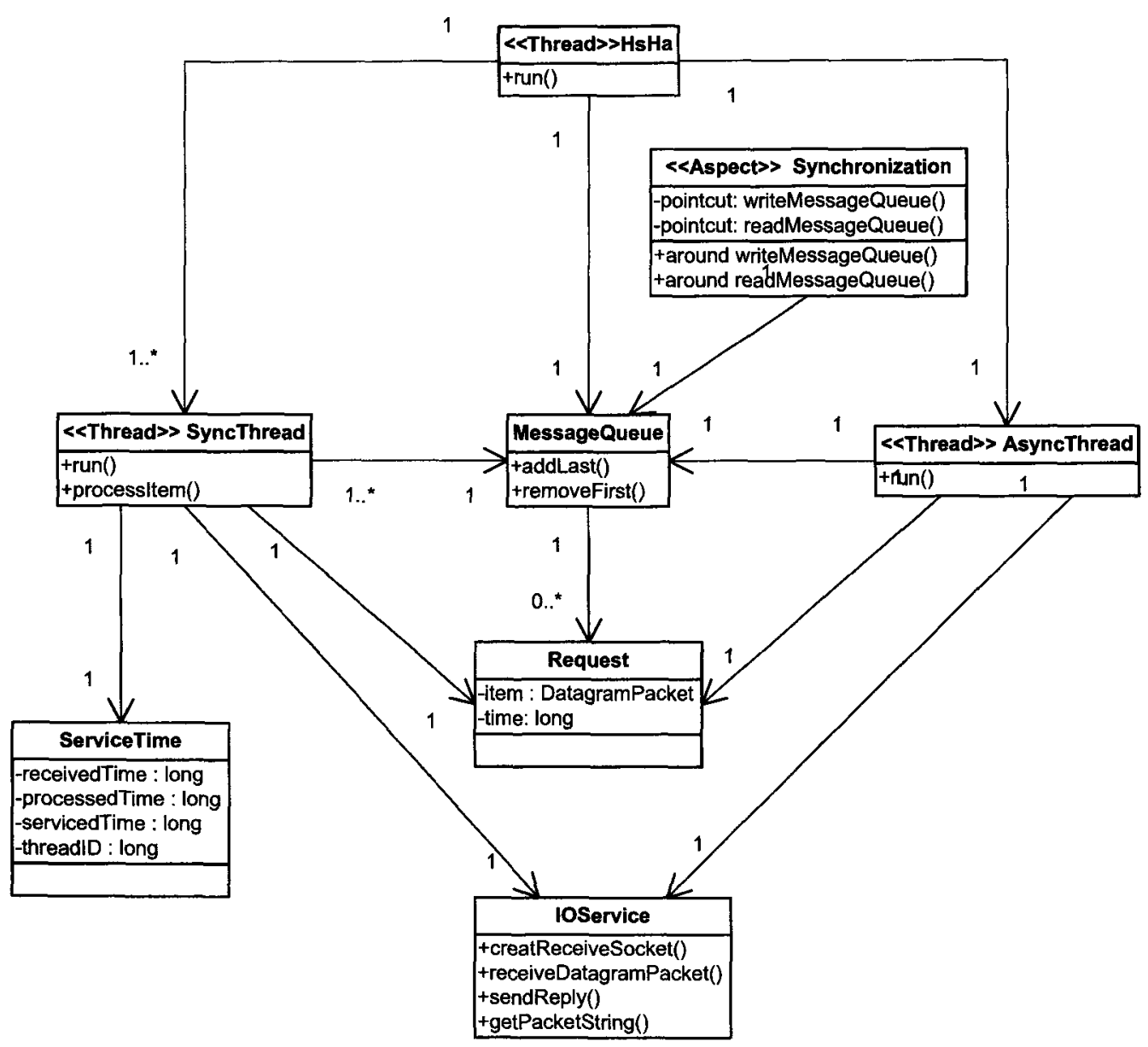

Figure 3.7 Class diagram of HS/HA with Aspect enabled

Synchronization is a class of stereotyped aspect, which consists of two pointcuts and two advices. These two pointcuts pick up the join points when the message queue's public methods are invoked. Since these public methods of the message queue are called 
within some method body of either objects of SyncThread or AsyncThread, the pointcut description (PCD) has to be specific in order to capture the object that holds the right to using the message queue.

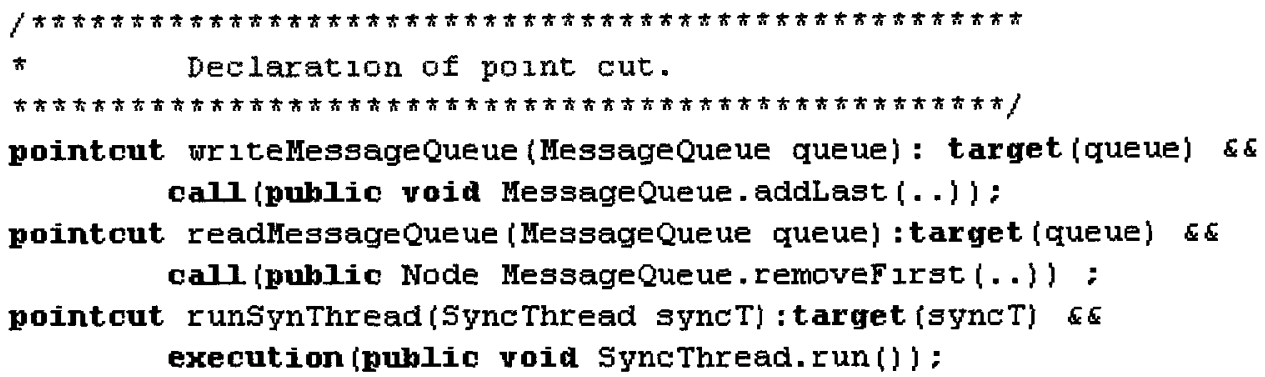

Figure 3.8 Pointcut description of HS/HA

Figure 3.8 illustrates the code portion of the Synchronization aspect. There are three PCDs shown in Figure 3.8. PCD writeMessageQueue picks up the call of public method addLast() of MessageQueue. This PCD also passes in queue as an argument to the advice, so that advice can do the necessary CPU-bound operation within itself. PCD readMessageQueue in this case picks up the calls of removeFirst(). Similarly, it also passes in queue as an argument to perform necessary CPU-bound operations. The last PCD runSynThread()captures the object and calls upon the message queue's public method. In this case, the argument that got passed into PCD runSynThread() is of typed SyncThread. This PCD picks up join points within the execution of run() call in SyncThread objects. 


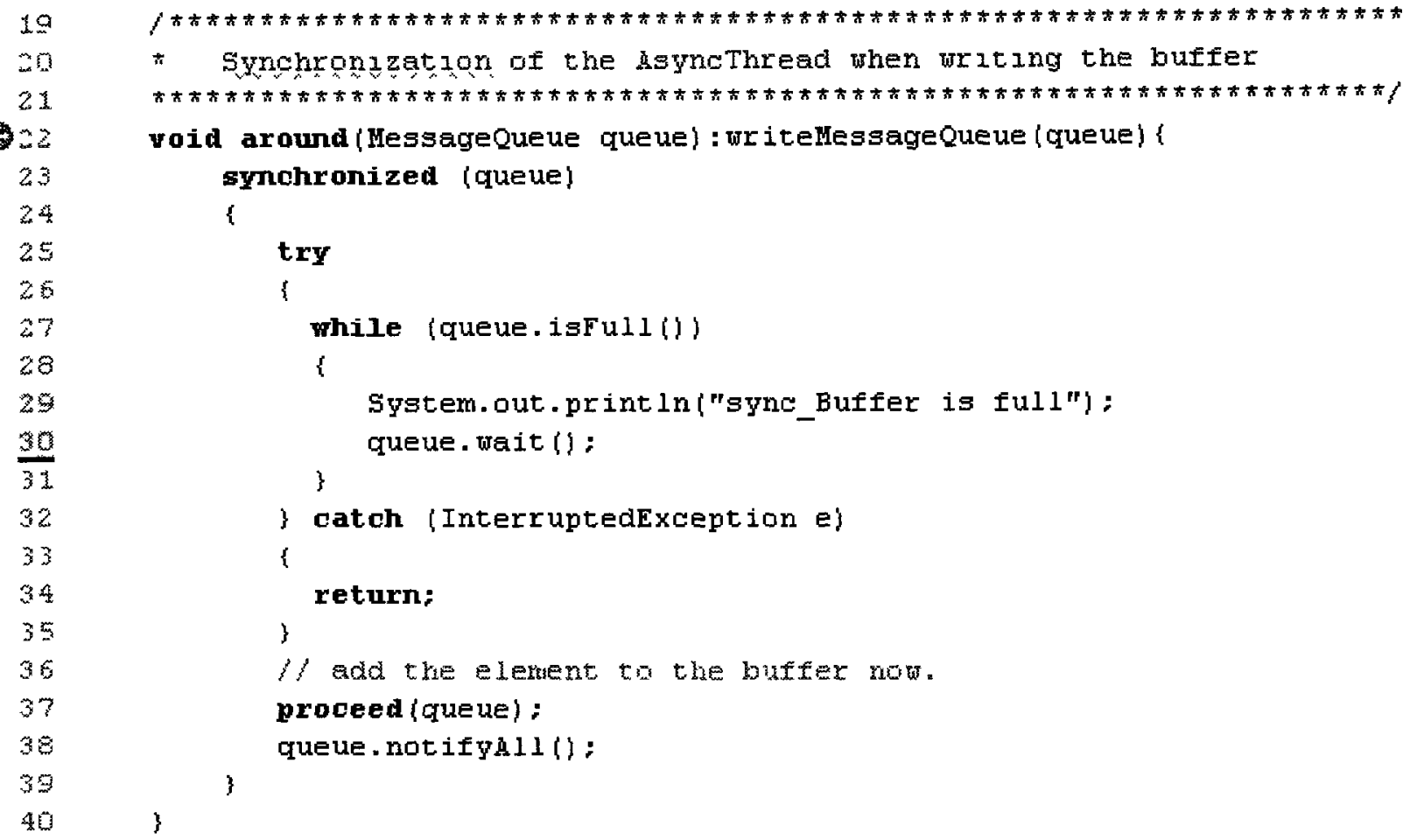

\section{Figure 3.9 Advice of HS/HA when writing the message queue}

The advice on writing the message queue is presented in Figure 3.9. This advice is constructed by an around advice upon a PCD writeMessageQueue(). This advice firstly locks the message queue queue using synchronized. Thus, no other synchronous threads can retrieve messages from the queue. This ensures data consistency. If the queue is full, advice will give up the lock on the queue, notify other threads to wake up and consume the messages that are stored in the message queue. Since the advice detects that the queue is not full anymore, it calls proceed(queue) and returns the program control back to the base program with an argument queue that was originally passed on to the advice. The 
advice then notifies all other non-active threads that are waiting their turn to access the message queue.

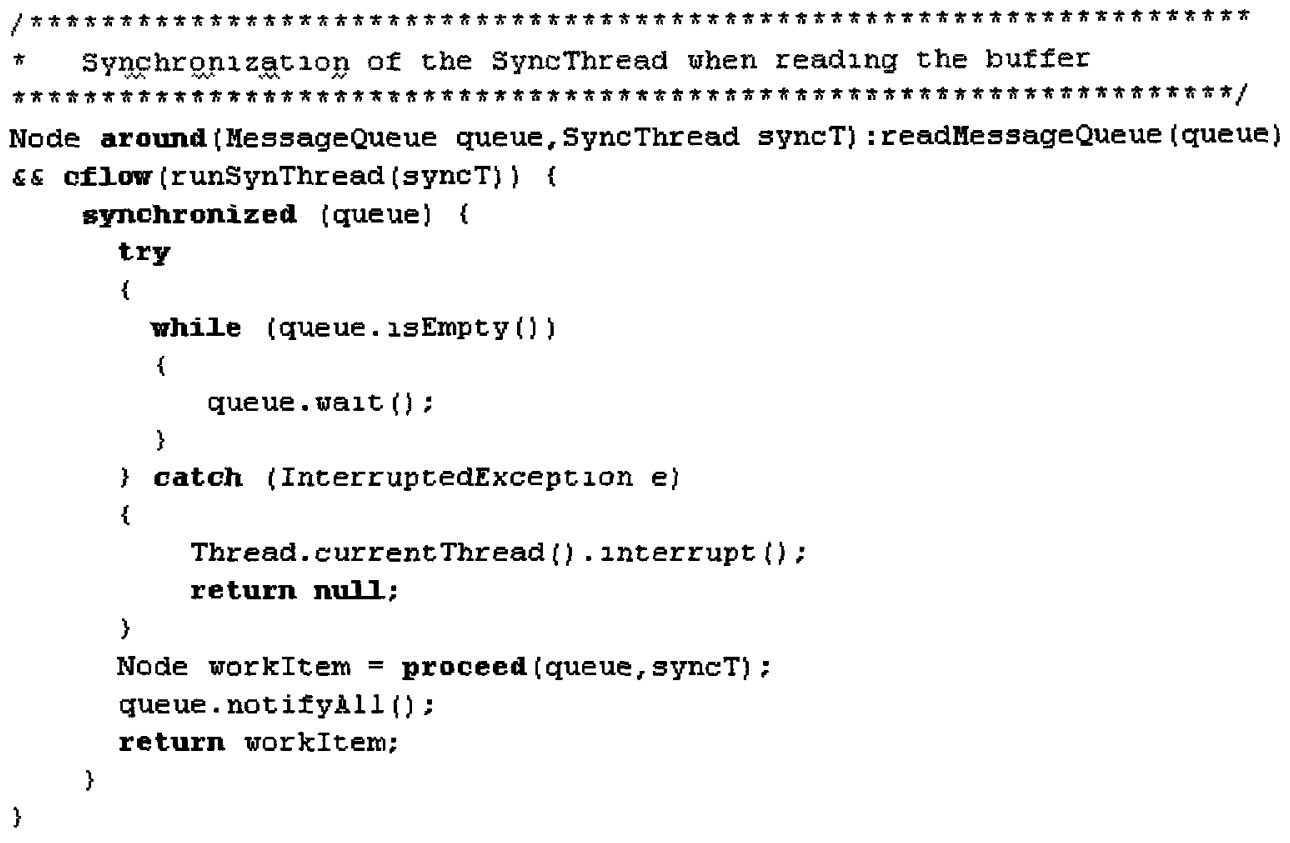

Figure 3.10 Advice of HS/HA when retrieving the message queue

The advice of retrieving a message from the message queue is presented in Figure 3.10. The advice code is constructed by an around advice upon a PCD readMessageQueue( and runSyncThread(). By using the Boolean operator, PCDs can combine to capture a more specific group of join points. Thus, this around advice only intervenes with the base program when accessing of the message queue takes place within the run() loop of a SyncThread object. The cflow() stands for control flow; it is an AspectJ specific construct of a primitive pointcut. With the help of cflow(), the PCD runSyncThread() defines that the advice will intervene only within the control flow of run() loop, and no other place. So the argument syncT (synchronous thread) can be 
accessed within the body of advice. AspectJ has another primitive which can extract the object where the join point is captured, which is thisJoinPoint. With this primitive, thisJoinPoint, the information of the join point can be retrieved at any place within the advice. However, the object retrieved by using thisJoinPoint can be of any type of instance object. Thus, using a PCD runSyncThread() can help to eliminate the join points from other anonymous object, calling on the public method of the message queue. The complete code of AspectJ and the classed they advised is presented in Appendix B.

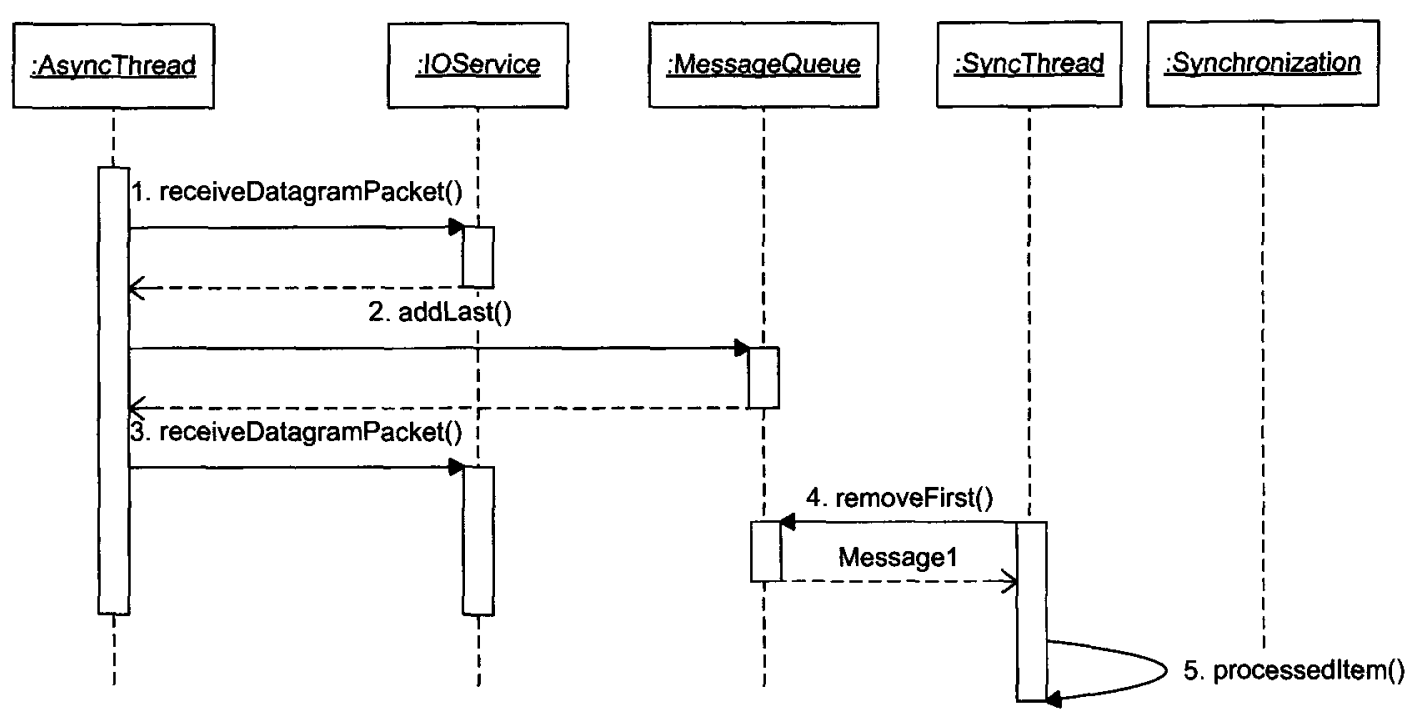

Figure 3.11 Sequence diagram of HS/HA with Aspect disabled

Figure 3.11 presents the sequence diagram of how the AOP can affect the performance impact during the switching of the control flow advices and base program. This sequence diagram of HS/HA has no aspect enabled. First, the asynchronous thread tries to access the $\mathrm{I} / \mathrm{O}$ operation in order to capture the incoming requests from clients, 
and then stores the request as an encapsulated packet in the message queue. The asynchronous thread continues to capture the incoming requests. The asynchronous thread can yield itself when the message queue is full, or wait for more requests to come in. The asynchronous thread yields the CPU resource to other threads (synchronous threads) in order to work on the packets that have been previously stored in the message queue. Once the message queue is not empty or no other threads are accessing it, one of the synchronous threads can retrieve a packet from the message queue and perform the CPU and emulated database operation accordingly.

In contrast to Figure 3.11 , Figure 3.12 illustrates the sequence diagram of HS/HA with aspect enabled. With the power of AOP paradigm, the Synchronization aspect uses the around advice to ensure the pre-conditions are made and post-conditions are satisfied whenever there are join points being picked up by the pointcuts description. The red circles that are shown in Figure 3.12 indicate the amount of extract time taken by the advice to complete the work and ensure the pre- and post-conditions are made before returning the control flow back to the base code. 


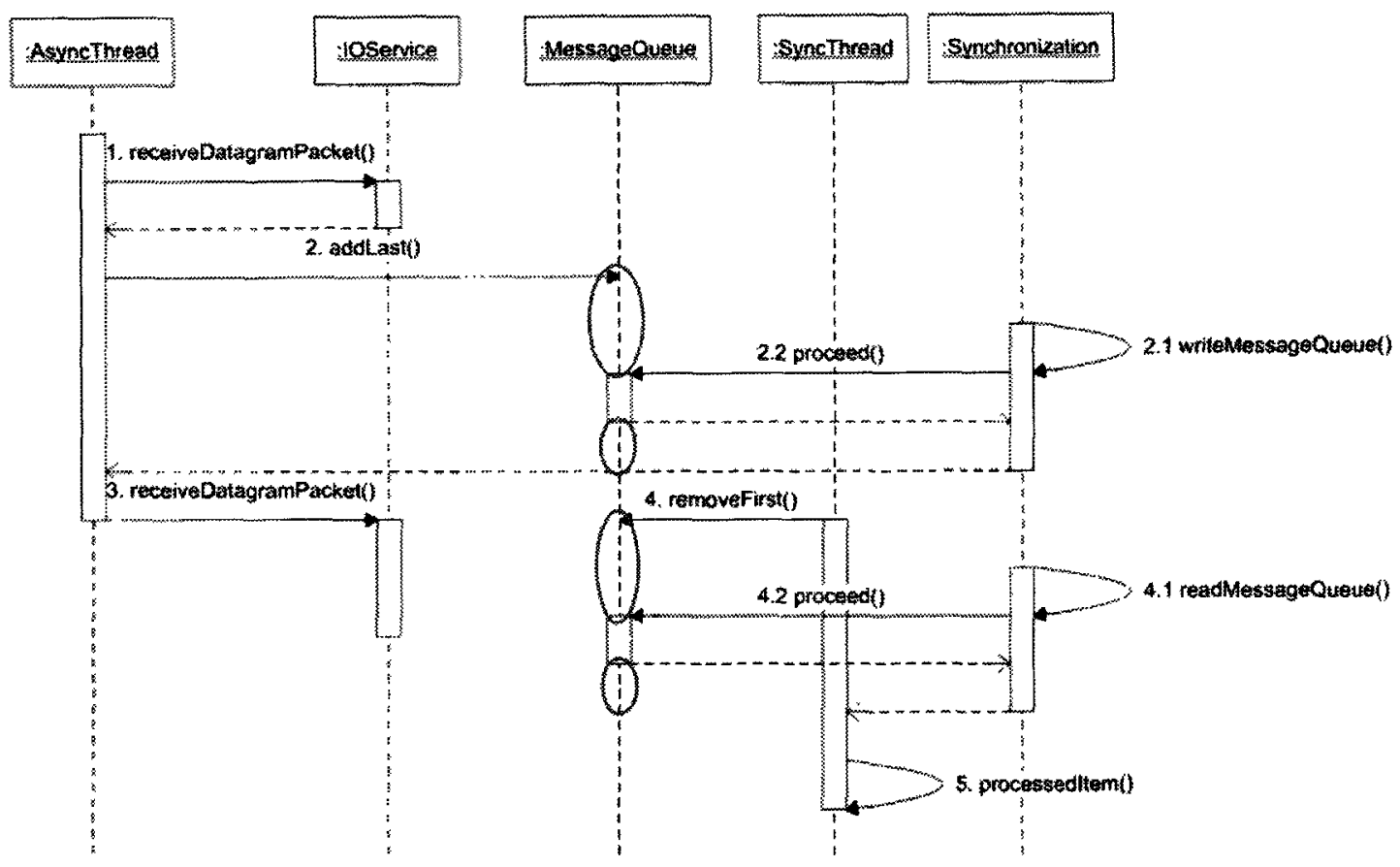

Figure 3.12 Sequence diagram of HS/HA with Aspect enabled

The following steps explain the execution sequence presented in Figure 3.12:

1. An AsyncThread object tries to receive the request coming from the client by invoking public method receiveDatagramPacket() on the class IOService.

2. Once the request is captured, it is stored into the MessageQueue object by invoking addLast(). The MessageQueue object works in a first-in-first-out fashion.

3. Method addLast() has been captured as a join point, and advice writeMessageQueue() is activated instead. The control flow of the program is now moved towards the aspect class. 
4. proceed() is called within the advice when the pre-conditions are made. The program's control flow returns back to the base program. The MessageQueue object is active now.

5. Once the method addLast() terminates, the MessageQueue object becomes inactive and the control flow is moved to the aspect class. The advice ensures the post-condition of addLast $($ and returns the control flow to the base program.

6. The AsyncThread object keeps on receiving for more incoming requests from the client.

7. Whenever the MessageQueue object is not empty, one of the SyncThread objects can gain access on the MessageQueue object and invoke removeFirst() in order to retrieve one request packet from the queue.

8. Method removeFirst 0 has been captured as a join point and the advice readMessageQueue( is activated instead. Once the advice ensures the preconditions of removeFirst(), proceed() is called within the advice. The control flow is returned back to the base program and the MessageQueue object is active.

9. When the removeFirst () terminates, the control flow is moved to the Synchronization aspect. The advice will ensures all the post-conditions of removeFirst(), and then moves the control flow from Synchronization to the SyncThread object. 
10. The SyncThread object goes on processing the request by invoking processedItem().

The red oval shaped circles represent the length of overhead when the control flow is moved between the base program and code of advices.

\subsection{Chapter Summary}

This chapter described the design of two existing software programs through a reverse engineering effort. These two existing systems are HS/HA concurrency architecture and LFs concurrency architecture. During the reverse engineering process of these two systems, concerns that cut across multiple classes are identified and extracted. These concerns were identified by the use of the concurrency control codes like synchronized, wait, notify and notifyAll [Sor07][Lea99]. New design using AOD and AOP was realized to simplify the concurrency control of the two original software systems under study. 


\section{Chapter 4 Experiments and Analysis}

This chapter outlines the details of the experiments conducted and presents the results. This chapter is organized as follows. Section 4.1 describes the physical environment of the experiments, the hardware, and network facilities. Section 4.2 describes how the performance measurements have been conducted. Section 4.3 illustrates the parameters and invariants that should hold. Sections 4.4 and 4.5 present final results of all the parameters tested and the performance impact of aspect-oriented design and implementation. Finally, the overall results of the experiments are summarized.

Essentially, the major focus of these experiments is to measure the performance impact of AOP paradigm based on two hypotheses:

1. The performance of the base code is somewhat degraded when AOP approach is applied to the base application software.

2. The incremental effect on performance becomes more obvious as the number of join points increases.

Programmers and software developers may choose the AOP paradigm for better maintainability and modularity, or simply the traditional OOP without AOP features because of performance reasons. The results presented in this work are not meant to advocate for AOP paradigm, but to investigate the impact of using AOP versus OOP in the area of concurrent programming. 


\subsection{Experiment Settings}

The experiments were carried out under an emulated multi-tier system environment as illustrated in Figure 4.1. The server simply receives the requests sent by multiple clients across the network and replies accordingly. Due to the constraints of hardware resources, these multiple clients are emulated by a single computer with multiple threads created in order to mimic the behaviors of multiple clients in a multi-tier system. Each client thread has its role in the network's traffic for its short bursts of request sending to the server.
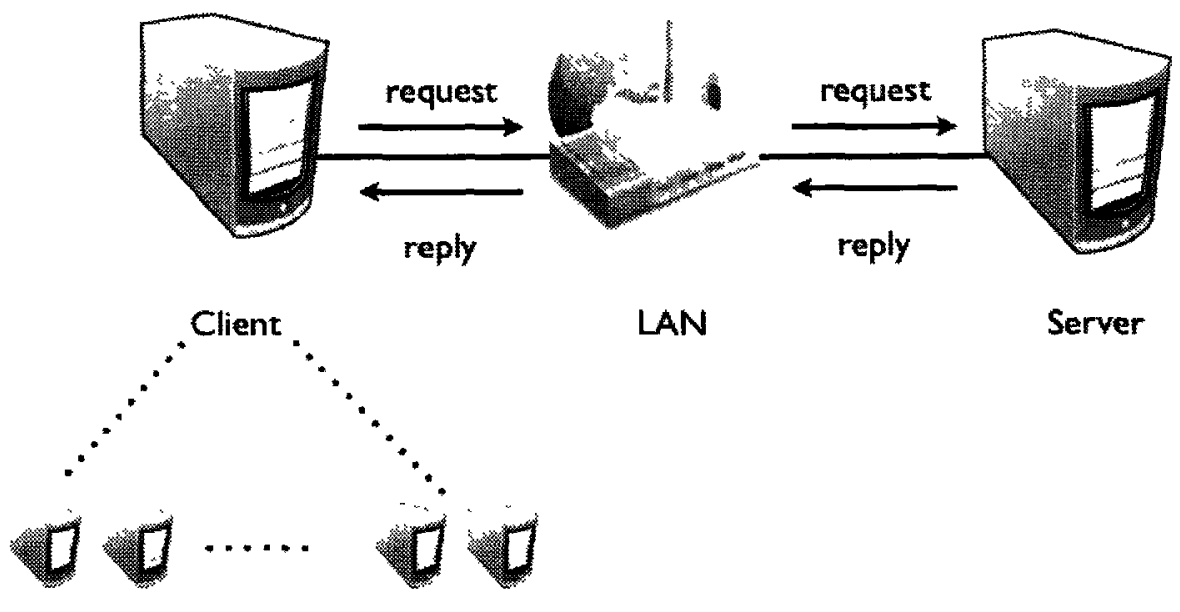

LAN

Server

Emulated Multiple Clients

Figure 4.1 Emulated multi-tier system of the experiment setup [Zha10a]

Figure 4.2 presents the hardware specifications of the server machine, which are obtained from CPU-Z [CPU10]. The server machine is composed of $2.4 \mathrm{GHz}$ Intel Core 2 Quad CPU with 3GB of RAM. The client machine which is used to emulate the multiple clients is a Macbook, with $2 \mathrm{GHz}$ Intel Core 2 Duo CPU with $1 \mathrm{~GB}$ of RAM. These two machines are connected through a local area network (LAN) in the Systems \& Computer 
Engineering department. The server side software is SUN's JDK Version 6 with JAVA platform running on Microsoft XP. The client side is set to JAVA SE 6 with a Mac Leopard operation system.
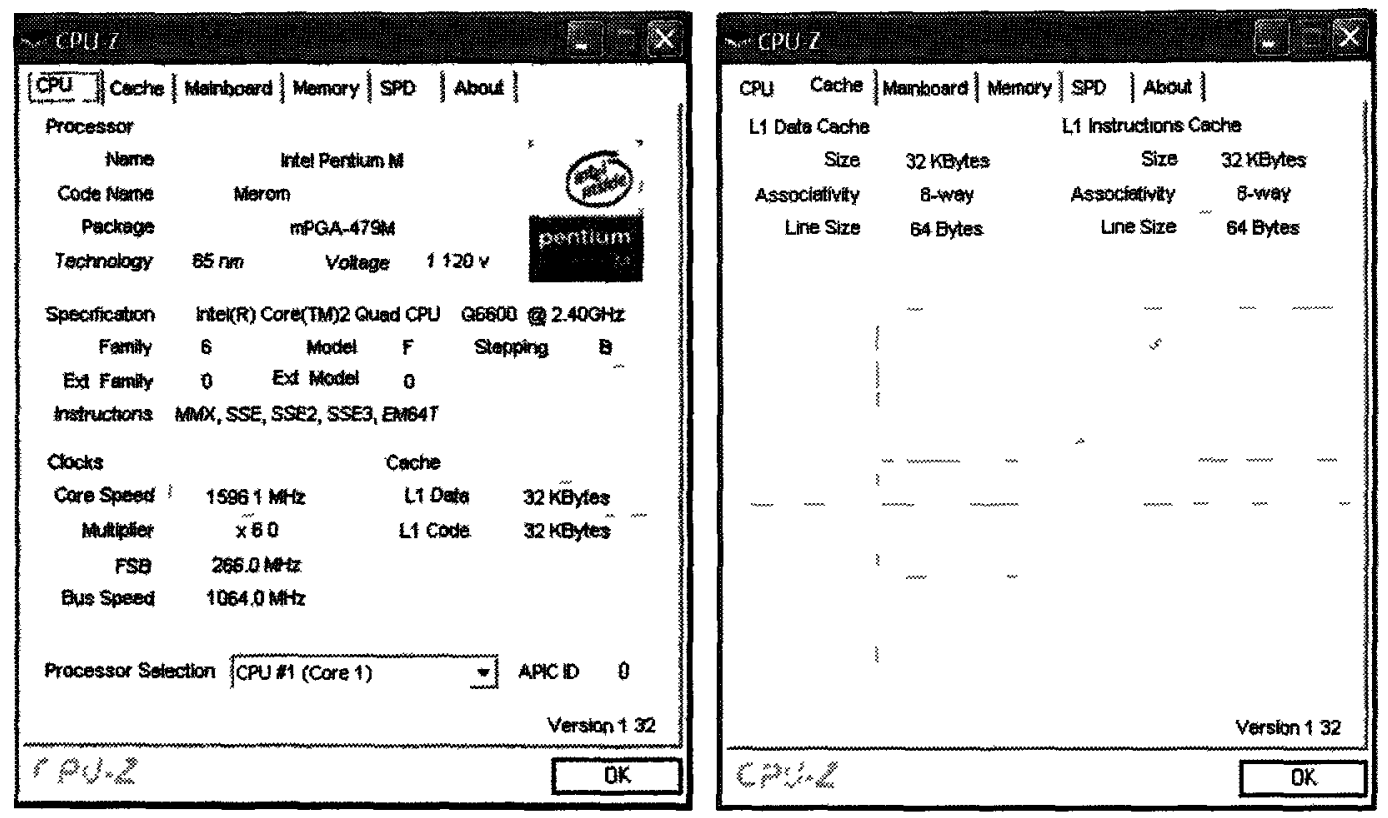

Figure 4.2 CPU and cache specification of the server machine

Figure 4.3 illustrates the emulated scenario on the server side by mimicking the I/O bound operations [Oso07]. The hypothetical I/O bound operations are emulated by invoking a Java thread sleep ( method to stop the CPU cycle and yield the CPU resources to other threads while waiting for the return of the sleep() call [Oak04]. Since there is no consumption of CPU while a thread goes to sleep, this is considered as the I/O bound operation while processing the requests [Wel00]. 


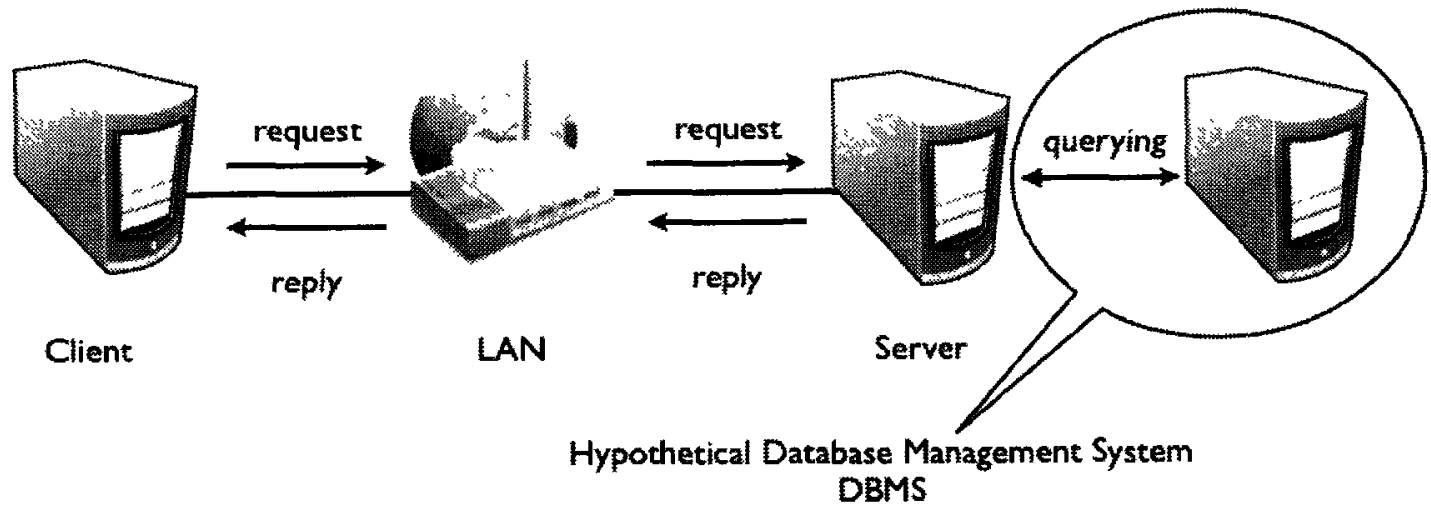

Figure 4.3 Emulated I/O bound operation in request processing [Oso07] [Zha10a]

\subsection{Performance Measurements}

This section presents different measurements considered on this experiment at the server side.

1. Average Service Time - This is the average service time of multiple runs of 32,000 packets served at the server side.

2. Performance Impact of AOP program - This is a measurement of the performance overhead of the AOP-based program compared to the non-AOP program. This is calculated as follows:

Average Service Time with AOP Disabled * Average Service Time with AOP Enabled Average Service Time with AOP Disabled $\times 100 \%$ (Eq. 1)

This value can either be positive or negative. A positive value indicates that the AOP based program runs faster than the non-AOP program, and vice versa.

3. System Throughput - It represents the number of requests processed per second. This is defined as: 
Total number of packet processed

Total elapse time

Packets continuously arrive at the server when the first packet arrives. The total elapse time is the gap between the arrival time of first packet served and the departure time of last packet served at the server side.

\subsection{Experiment Parameters}

The following parameters are varied in both HS/HA and LFs and are evaluated with either AOP paradigm enabled or disabled. Different experiment settings have been conducted and a tabulated set of results are presented in later sections. The details of the parameters are described as follows:

1. Thread Pool Size - This parameter refers to the total number of threads allocated in LFs architecture, or the total number of threads allocated to become the synchronous threads in the synchronous layer of HS/HA architecture.

2. Queue Size - This parameter only applies to HS/HA architecture where the queuing layer resides. This is the critical section where both the synchronous and asynchronous layers compete for the accessing right. It is also called message queue in this experiment.

3. Number of join points - This parameter is used to manually increase or decrease the number of join points by adding some custom-made method invocations to be captured by the PCDs. These additional methods added to the base code do not alter nor modify the functional behavior of the base application software. 
4. CPU only or CPU- and IO-Bound operation - The previous three parameters will be evaluated under the CPU-bound only environment and a hybrid environment of CPU- and IO-bound. Emulated database operations are to mimic the V/O-bound operations as shown in Figure 4.3.

\subsection{Performance Evaluation of AOP Using LFs}

There is one intrinsic parameter of LFs, which we can vary, i.e., the size of the thread pool. The size of the thread pool also defines the number of threads created in the system. Setting this value too high will lead to low CPU utilization and setting it too low will cause long response times instead [Zha10a]. The main idea of the experiment is to investigate the performance impact of AOP paradigm. Thus we will manually vary and add join points to measure and analyze the performance impact of AOP.

\subsubsection{Performance Evaluation of AOP Using LFs with Different Thread Numbers in a CPU-Bound Operating Environment}

This section demonstrates the phenomena of changing the number of threads created in the thread pool in terms of the service time at the server side. The service time is defined as the average amount of time it takes to process the requests at the server side. This average service time is measured by processing multiple runs of 32,000 packets in a row.

Figure 4.4 illustrates the average service time with CPU-bound operations. Looking at threads 5-160, the difference between AOP and non-AOP is not significant. But as the number of threads allocated to the thread pool increases to 640 or 5000 , the 
application software program with AOP paradigm enabled runs even faster than the base program with AOP-disabled.

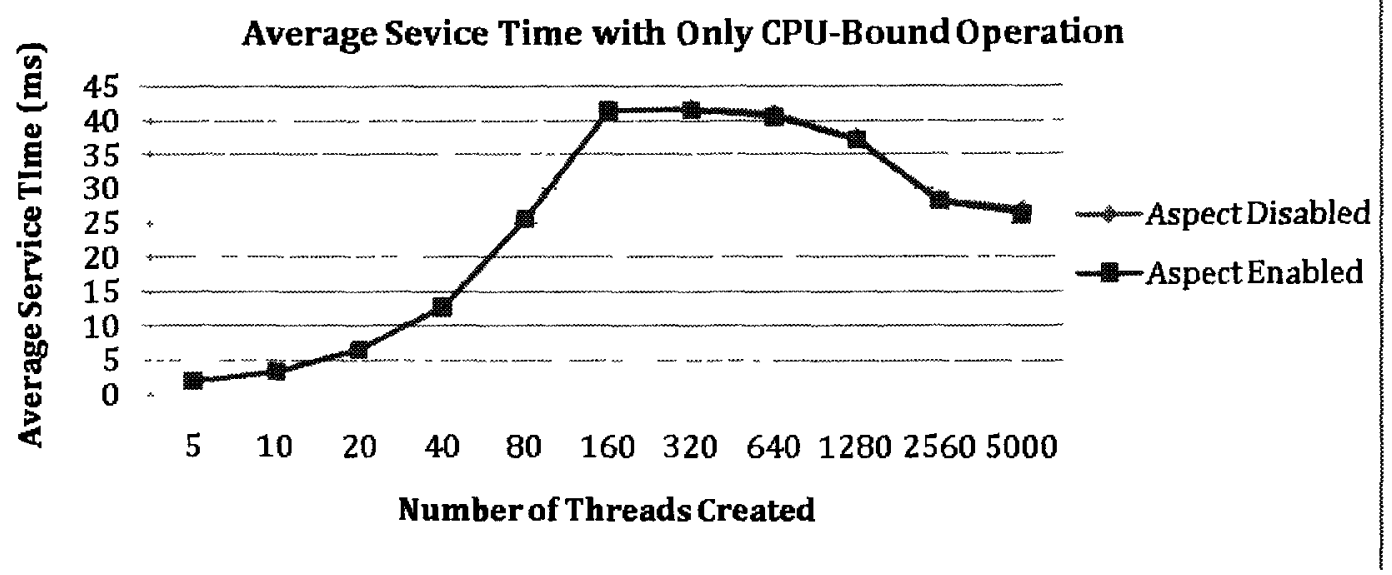

Figure 4.4 Average service time of AOP-enabled and AOP-disabled using LFs

The Performance Impact of AOP program is shown in Table 4.1 which presents the detailed results of how AOP-based program performs compared with AOP-disabled software. Based on Eq. 1 in Section 4.2, a negative value indicates that the AOP based program runs slower than the base application program and a positive value means that the AOP-based program runs faster. With the help of what Table 4.1 presents, it can be observed when the hypothesis stands and when it fails. At thread 80, AOP-disabled program uses $25.30 \mathrm{~ms}$ of average service time, but AOP-enabled uses 25.52. This indicates AOP-enabled needs more time to process a request. But for other numbers of threads, AOP-enabled software is faster than AOP-disabled base program. 
Table 4.1 Average service time of with different thread numbers created

\begin{tabular}{|l|c|c|c|c|c|c|c|c|c|c|c|}
\hline No. of & 5 & 10 & 20 & 40 & 80 & 160 & 320 & 640 & 1280 & 2560 & 5000 \\
\hline $\begin{array}{l}\text { Average } \\
\text { Service } \\
\text { Time } \\
\text { with Aspect } \\
\text { Disabled } \\
\text { (ms) }\end{array}$ & 2.00 & 3.45 & 6.60 & 12.88 & 25.30 & 41.50 & 41.85 & 41.13 & 37.49 & 28.34 & 27.00 \\
\hline $\begin{array}{l}\text { Average } \\
\text { Service Time } \\
\text { with Aspect } \\
\text { Enabled } \\
\text { ( m s })\end{array}$ & 1.99 & 3.43 & 6.57 & 12.78 & 25.52 & 41.44 & 41.51 & 40.53 & 37.19 & 28.09 & 26.34 \\
\hline $\begin{array}{l}\text { Performance } \\
\text { Impact of } \\
\text { AOP } \\
\text { program (\%) }\end{array}$ & 0.54 & 0.60 & 0.47 & 0.75 & -0.87 & 0.14 & 0.81 & 1.45 & 0.81 & 0.88 & 2.44 \\
\hline
\end{tabular}

After careful analysis, the AOP-based program running faster is due to the low cache fault data. In other words, whenever the OS has low cache fault/second, the program provides better performance results. The cache fault data were collected by using the built-in Performance Logs and Alerts tool from Microsoft Window (XP Professional) [Ms06], and the average values of multiple runs are presented in Table 4.2. 
Table 4.2 Cache fault/second with different number of threads created

\begin{tabular}{|l|c|c|c|c|c|c|c|c|c|c|c|}
\hline No. Threads & 5 & 10 & 20 & 40 & 80 & 160 & 320 & 640 & 1280 & 2560 & 5000 \\
\hline $\begin{array}{l}\text { Cache } \\
\text { fault/second } \\
\text { with } \\
\text { Aspect } \\
\text { Disabled }\end{array}$ & 4089 & 3004 & 3733 & 3592 & 3935 & 2524 & 2494 & 1201 & 977 & 1513 & 569 \\
\hline $\begin{array}{l}\text { Cache } \\
\text { fault/second } \\
\text { with } \\
\text { Aspect } \\
\text { Enabled }\end{array}$ & 3007 & 2016 & 3601 & 3445 & 4559 & 2387 & 1932 & 1016 & 811 & 1040 & 515 \\
\hline $\begin{array}{l}\text { Performance } \\
\text { Impact of } \\
\text { AOP } \\
\text { program (\%) }\end{array}$ & 0.54 & 0.60 & 0.47 & 0.75 & -0.87 & 0.14 & 0.81 & 1.45 & 0.81 & 0.88 & 2.44 \\
\hline
\end{tabular}

The data presented in Table 4.2 are not exactly reproducible, which means the measurements are not expected to be exactly the same for the same experiment if executed again. Caching is highly dependent on the management of cache memory and users do not have control of it. The fact that AOP program runs faster than non-AOP is different from general conception which is that AOP has some performance overhead. Even though when the AOP-based program does not run faster than the non-AOP program, the performance impact is small compared to the potential gain in modularity enhancement.

Figure 4.5 illustrates when the AOP-based program runs faster. This graph is based on the data presented in Table 4.1. It is observed that the AOP-based program does not have much performance impact on degrading the system. And in fact, AOP-based program even runs faster in most cases. The observations are only based on the 
experimental results of this particular system; further analysis should be conducted for other systems to get more generalized results.



Figure 4.5 Performance impact of AOP-based program compared with non-AOP based program

The system throughput of both the AOP based program and non-AOP based program are almost the same as illustrated in Figure 4.6. It is observed that the overall system throughput is degraded when the system is over-burdened with too many thread resources allocated. When a higher number of threads are allocated, thread contention and overhead cause the degradation of system throughput. Moreover, the value 5000 threads are allocated instead of 5120 is because the Eclipse system crashes, Figure 4.6 explains the reason. 


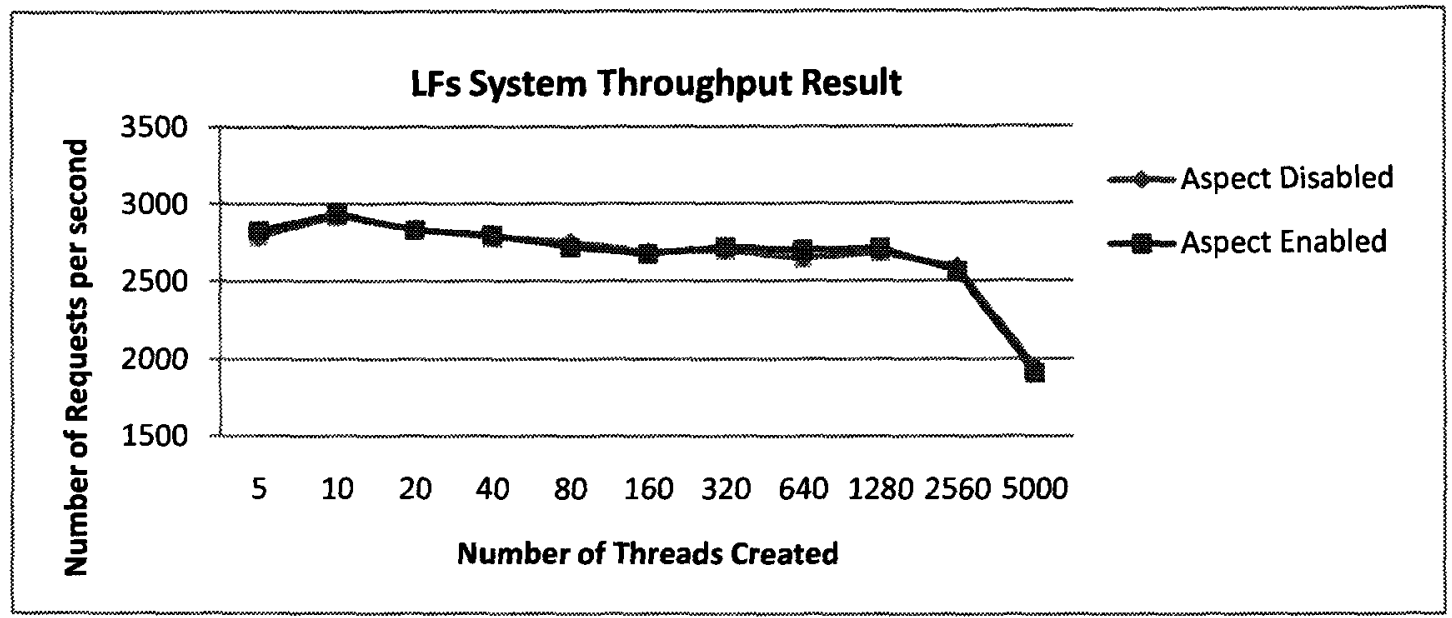

4.4.2 Figure 4.6 System throughput based on Table 4.1Performance Evaluation of AOP Using LFs with Different Number of Threads in a CPUand I/O-Bound Operating Environment

This section includes the emulated $\mathrm{V} / \mathrm{O}$-bound operations that use the thread sleep() method call to engage the thread in the running state. Since there is some extent of CPU-bound operations during the process of capturing the request from the socket, it is almost impossible to have a pure $\mathrm{I} / \mathrm{O}$-bound operations testing environment. The sleep() method call will cause the calling thread to sleep 100 milliseconds (ms), and the results are illustrated in Figure 4.7. 


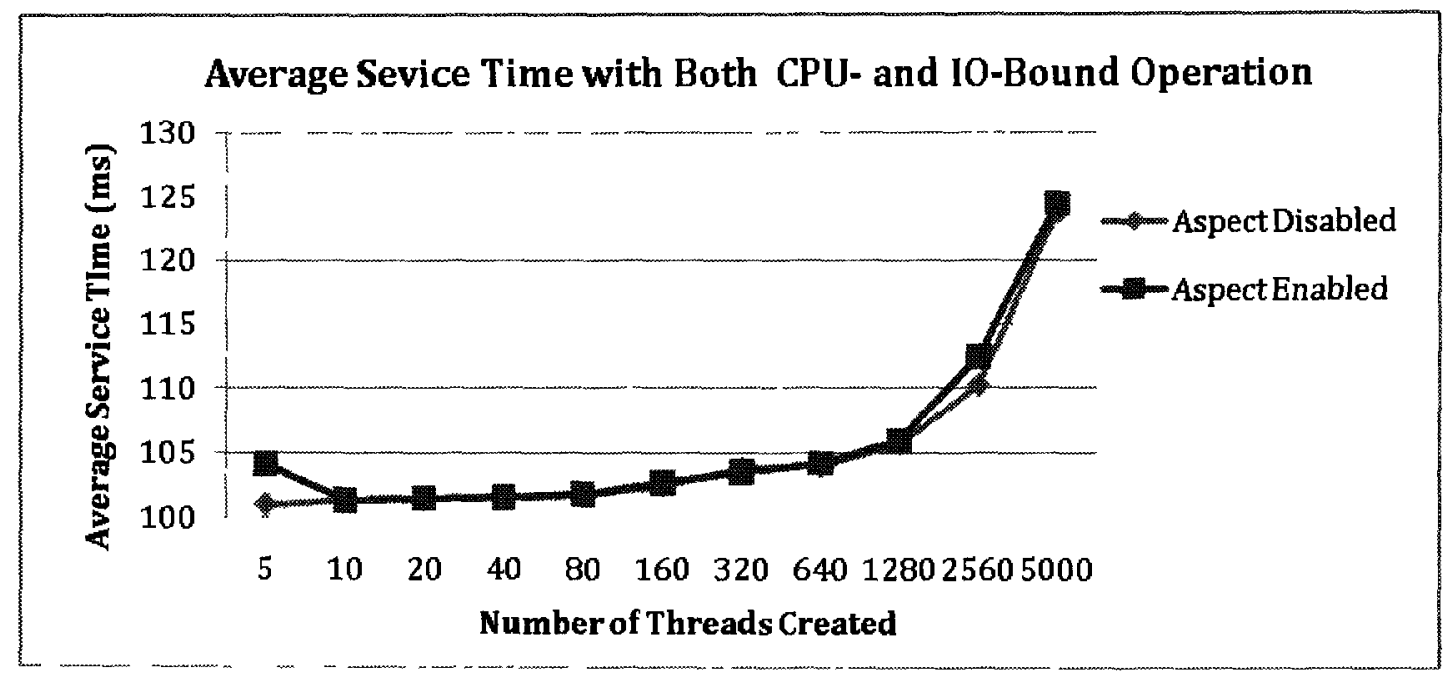

Figure 4.7 Average service time of LFs with $100 \mathrm{~ms}$ of I/O-bound operation

The data plotted inFigure 4.7 is presented in Table 4.3. It is average service time which represents the total time of CPU- and emulated I/O-bound operation. Since the sleep() call is set at $100 \mathrm{~ms}$, the time taken for CPU-bound operations can be calculated by subtracting $100 \mathrm{~ms}$ from the average service time as shown in Figure 4.7 or Table 4.3. 
Table 4.3 Average service time with different thread number created

\begin{tabular}{|l|c|c|c|c|c|c|c|c|c|c|c|}
\hline No. Threads & 5 & 10 & 20 & 40 & 80 & 160 & 320 & 640 & 1280 & 2560 & 5000 \\
\hline $\begin{array}{l}\text { Average } \\
\text { Service } \\
\text { Time } \\
\text { with } \\
\text { Aspect } \\
\text { Disabled } \\
\text { (ms) }\end{array}$ & 101 & 101.3 & 101.5 & 101.5 & 101.7 & 102.4 & 103.7 & 104 & 105.7 & 110.2 & 123.8 \\
\hline $\begin{array}{l}\text { Average } \\
\text { Service } \\
\text { Time } \\
\text { with } \\
\text { Aspect } \\
\text { Enabled } \\
\text { (ms) }\end{array}$ & 104 & 101.2 & 101.4 & 101.4 & 101.8 & 102.6 & 103.5 & 104.2 & 105.9 & 112.4 & 124.4 \\
\hline $\begin{array}{l}\text { Performance } \\
\text { Impact of } \\
\text { AOP } \\
\text { program (\%) }\end{array}$ & -3.15 & 0.047 & -0.05 & 0.005 & -0.06 & -0.24 & 0.184 & -0.2 & -0.25 & -2.05 & -0.50 \\
\hline
\end{tabular}

Table 4.4 presents the cache fault data of Figure 4.7 above.

Table 4.4 Cache fault/second of LFs with different number of threads created under CPU- and IO-bound operation

\begin{tabular}{|l|c|c|c|c|c|c|c|c|c|c|c|}
\hline No. Threads & 5 & 10 & 20 & 40 & 80 & 160 & 320 & 640 & 1280 & 2560 & 5000 \\
\hline $\begin{array}{l}\text { Cache } \\
\text { fault/second } \\
\text { with Aspect } \\
\text { Disabled }\end{array}$ & 33.5 & 34.5 & 34.4 & 37.6 & 48.5 & 45.8 & 45.2 & 33.8 & 41.1 & 55.2 & 44.0 \\
\hline $\begin{array}{l}\text { Cache } \\
\text { fault/second } \\
\text { with Aspect } \\
\text { Enabled }\end{array}$ & 33.9 & 31.9 & 31.4 & 37.2 & 56.2 & 49.4 & 40.7 & 42.8 & 42.5 & 56.7 & 49.8 \\
\hline $\begin{array}{l}\text { Performance } \\
\text { Impact of } \\
\text { AOP } \\
\text { program (\%) }\end{array}$ & -3.15 & 0.04 & 0.04 & 0.00 & -0.06 & -0.24 & 0.18 & -0.20 & -0.25 & -2.05 & -0.50 \\
\hline
\end{tabular}


Figure 4.8 illustrates the performance impact of AOP vs. non-AOP based programs. AOP-enabled software is a little bit faster than the base program in some cases. It is observed that overall the AOP-based program has very similar performance when an emulated $\mathrm{I} / \mathrm{O}$ operation is added to each request.

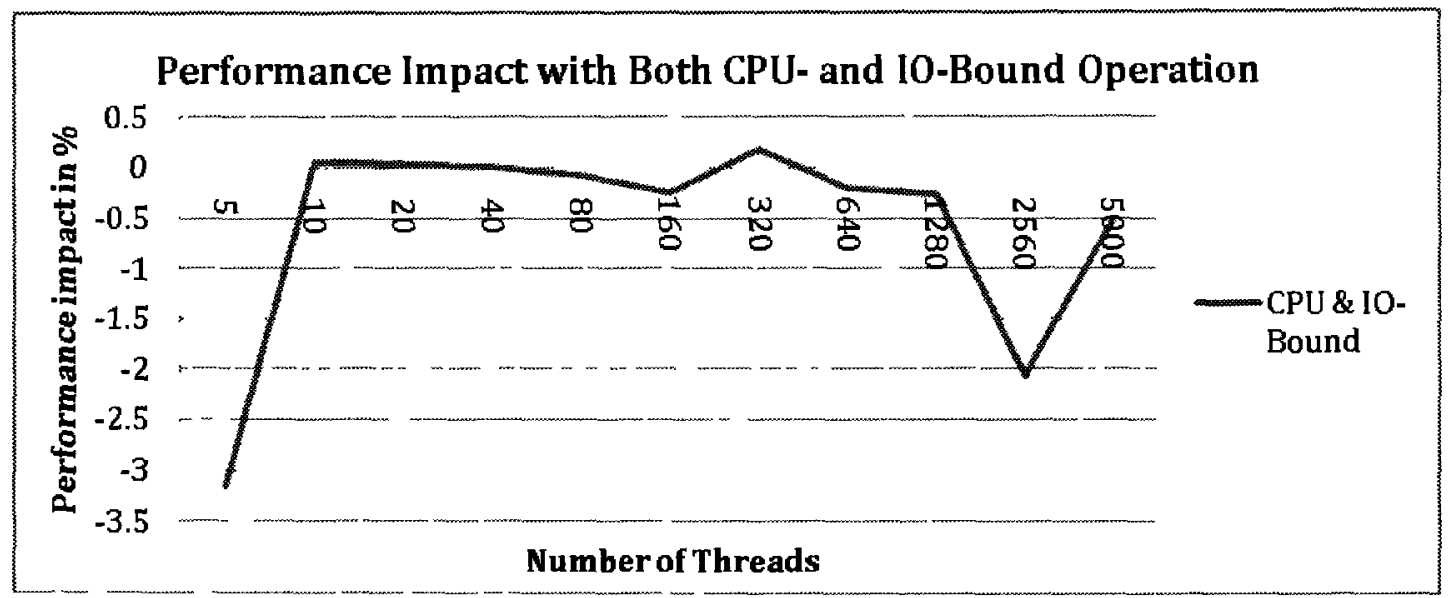

Figure 4.8 Performance of AOP-based program compared with the base program

Figure 4.11 illustrates the system throughput based on Table 4.3 with an I/Obound operation of $100 \mathrm{~ms}$ for each packet. Similar to the results presented in section 4.4.1, the system throughput of both the AOP and non-AOP programs are very close to each other as observed from the figure. 


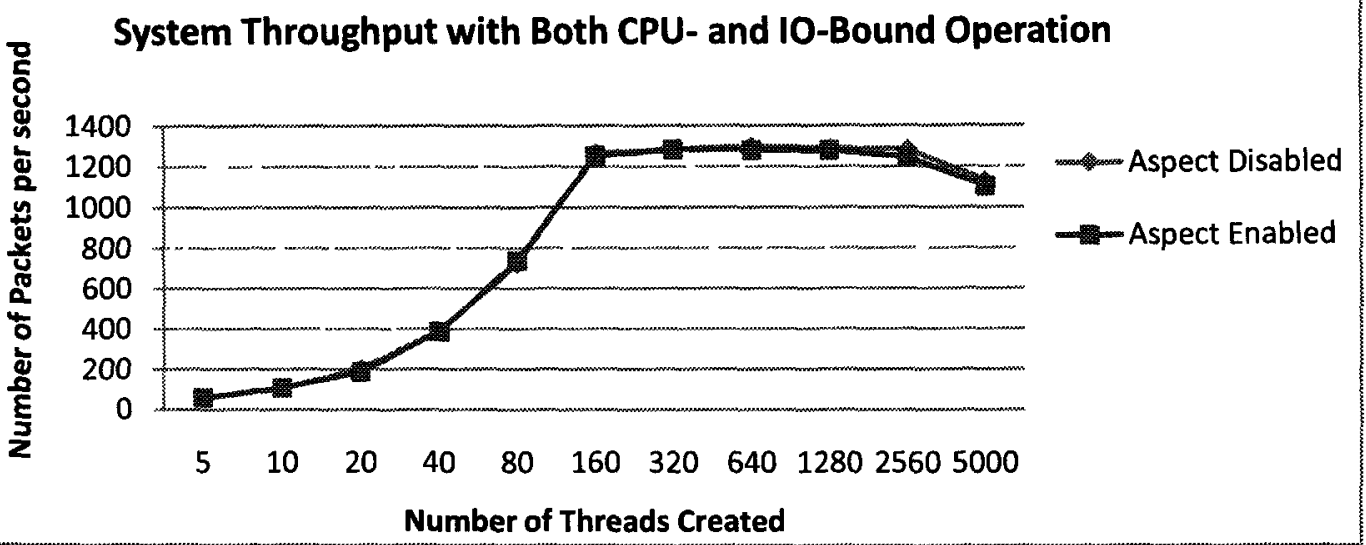

Figure 4.9 Comparison of system throughput of both CPU and I/O operation

\subsubsection{Performance Evaluation of AOP Using LFs with Different Number of Join Points in a CPU-Bound Operating Environment}

One of the aims of this research is to find the impact of switching control flow back and forth between the base program and the aspect module as shown in Figure 3.5. Theoretically, the additional switching of control flow should contribute additional CPU cycles, thus more time will be taken than just having the base program running without invoking the aspect code. However, the results shown in Section 4.4.1 suggest that the program with aspects can even run faster than the base application software. This section presents the results of additional join points that are manually added to the base program and using the aspect's pointcut descriptions (PCDs) to identify these method calls as join points. The additional method calls however do not alter the functionality of the program; they simply perform some dummy operations to consume some CPU time; for instance, 
printing out a message on the console. As shown in the previous section, both CPUbound and emulated $\mathrm{I} / \mathrm{O}$-bound operations are considered in this environment.

Figure 4.10 illustrates the average service time. The invariants in this case are the number of threads which is fixed at 160 and CPU-bound operations. The method calls are manually added to the base program. These method calls do not alter the functionalities of the original software, but serve only as join points which are later captured by PCDs whenever AOP is enabled. Similar to Sections 4.4.1 and 4.4.2, AOP outperforms its nonAOP counterpart in many scenarios. The number of threads is fixed at 160 in this section of the experiment for the following reasons [Zha10a]:

- 160 threads are expected to be enough to handle the arrival rate of the request.

- It is the middle sample point of previous sections (Section 4.4.1 and 4.4.2).

- According to Figure 4.4, the average service time is at the peak for 160 threads. Selection of this point may reveal more performance impact. 


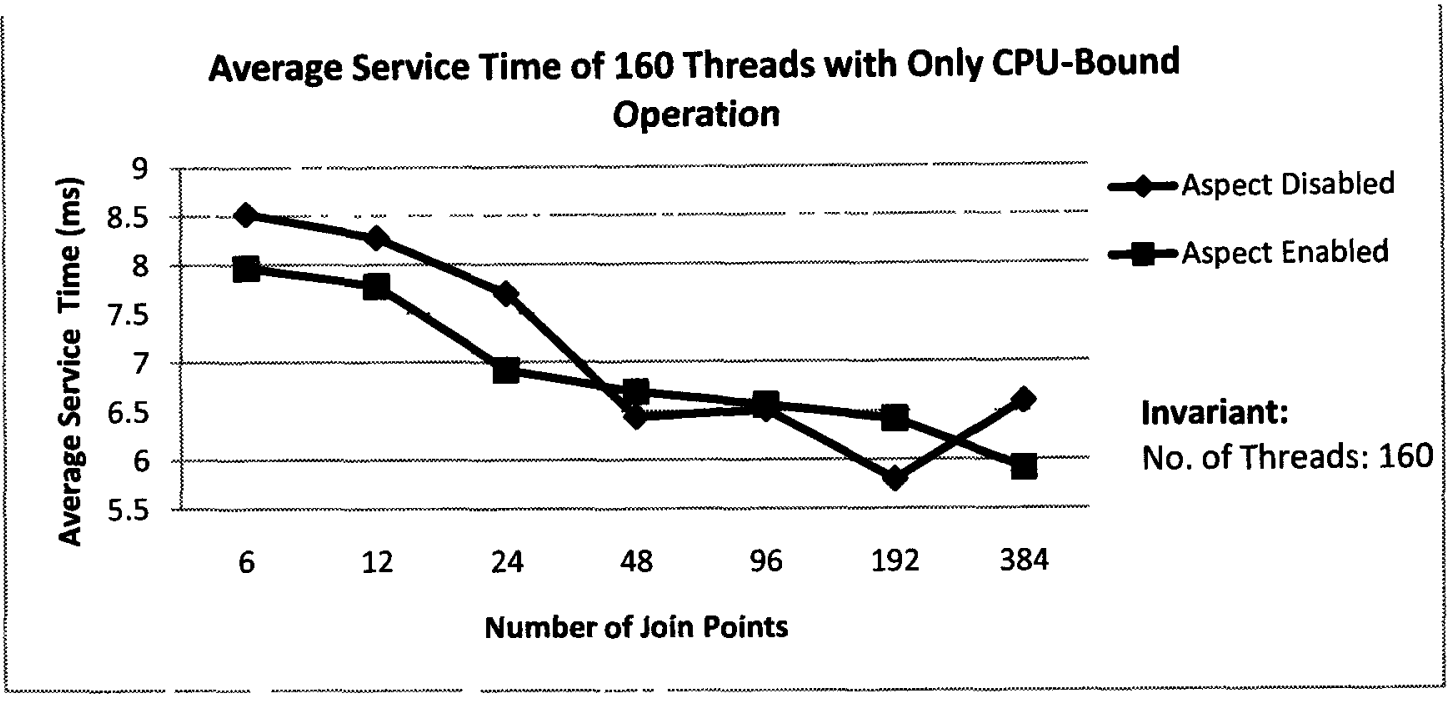

Figure 4.10 Average service time of a CPU-bound only operations for AOP-enabled and AOP-disabled programs w.r.t. number of joint points

Table 4.5 presents the cache fault data of Figure 4.10 above.

Table 4.5 Cache fault/second of LFs with different number of threads created under CPUbound operation

\begin{tabular}{|l|c|c|c|c|c|c|c|}
\hline No. Threads & 6 & 12 & 24 & 48 & 96 & 192 & 384 \\
\hline $\begin{array}{l}\text { Cache } \\
\text { fault/second } \\
\text { with Aspect } \\
\text { Disabled }\end{array}$ & 35.23 & 40.96 & 40.25 & 40.51 & 31.81 & 40.91 & 31.91 \\
\hline $\begin{array}{l}\text { Cache } \\
\text { fault/second } \\
\text { with Aspect } \\
\text { Enabled }\end{array}$ & 31.08 & 37.93 & 37.64 & 43.51 & 34.32 & 44.59 & 24.36 \\
\hline $\begin{array}{l}\text { Performance } \\
\text { Impact of } \\
\text { AOP } \\
\text { program (\%) }\end{array}$ & 6.467 & 5.947 & 10.12 & -3.98 & -0.73 & -10.6 & 10.34 \\
\hline
\end{tabular}


Figure 4.10 shows the performance impact of the number of method calls or join points. Theoretically speaking, having more join points means higher overhead. However, as Figure 4.11 illustrates, there is no trend or pattern of gradual decrease of performance spotted in the graph; instead, the figure shows that the AOP-enabled program can sometimes run up to $10 \%$ faster than AOP-disabled software. This happens sometimes because an AOP-based program has less cache faults than a non-AOP based program, which is dependent on the computing system. The result indicates that the performance impact could be closely tied to the memory management system instead of the aspect-oriented feature.

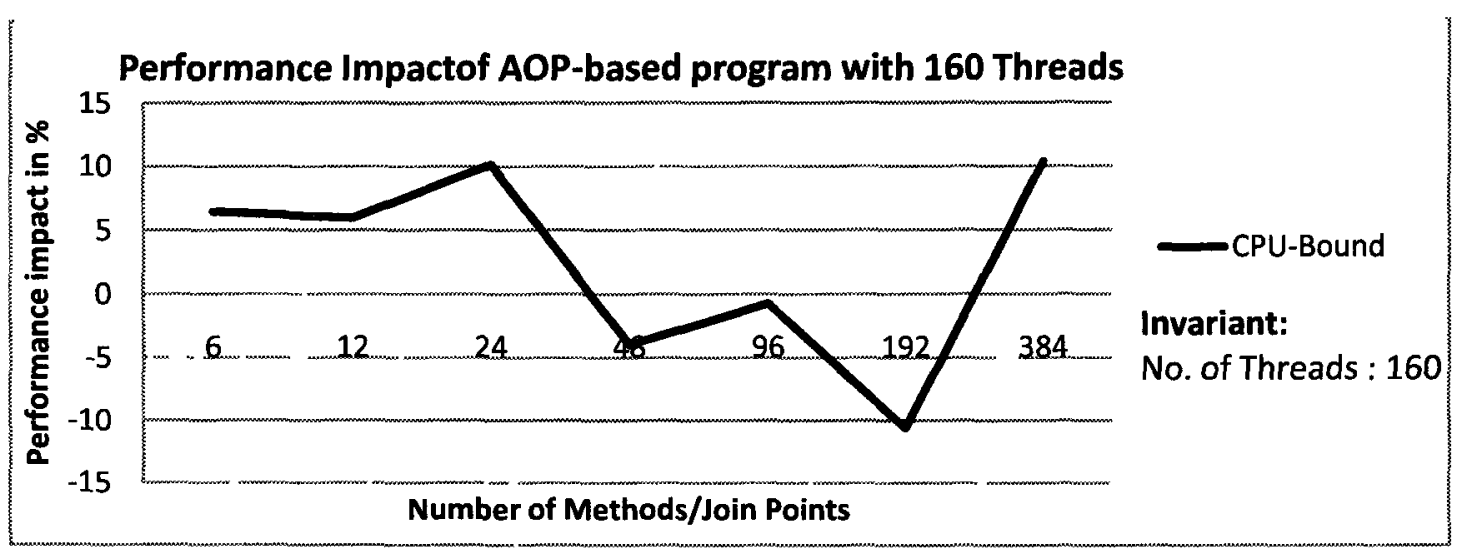

Figure 4.11 Performance impact of the AOP-based program

Figure 4.16 illustrates the system throughput with different number of join points. An interesting point observed from Figure 4.16 is when there are 192 methods/join points added manually. The system throughput is affected by the total elapsed. 


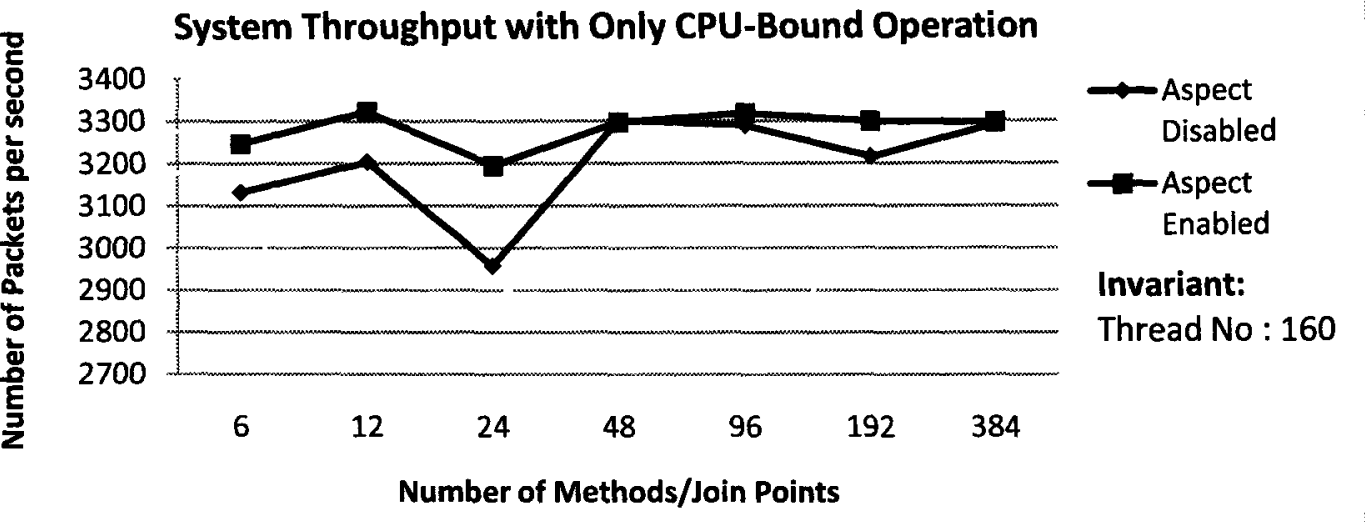

Figure 4.12 LFs system throughput of CPU-bound only operations for AOP-enabled and AOP-disabled programs w.r.t. number of joint points

\subsubsection{Performance Evaluation on AOP Using LFs with Different Number of Join Points in a CPU- and I/O-Bound Operating Environment}

Section 4.4.3 has presented the results of varying the number of join points in a CPU-bound only environment. In order to verify how AOP paradigm based program performs in different environment parameters, this section adds an emulated $\mathrm{I} / \mathrm{O}$ bound operation of $100 \mathrm{~ms}$ with 160 threads created.

Figure 4.13 illustrates the average service time of 32,000 packets being processed at the server machine with emulated $\mathrm{I} / \mathrm{O}$-bound operation. The invariant in this case is the number of threads which is allocated at 160 . As the number of methods/join points increases, the overall service time to process 32,000 packet should increase theoretically. However, such expectation was not observed. Moreover, the performance of the AOPbased program sometimes runs faster than the base application program as shown in 
Figure 4.13. These results are due to the cache fault data of AOP-based program which is low for the experiment.

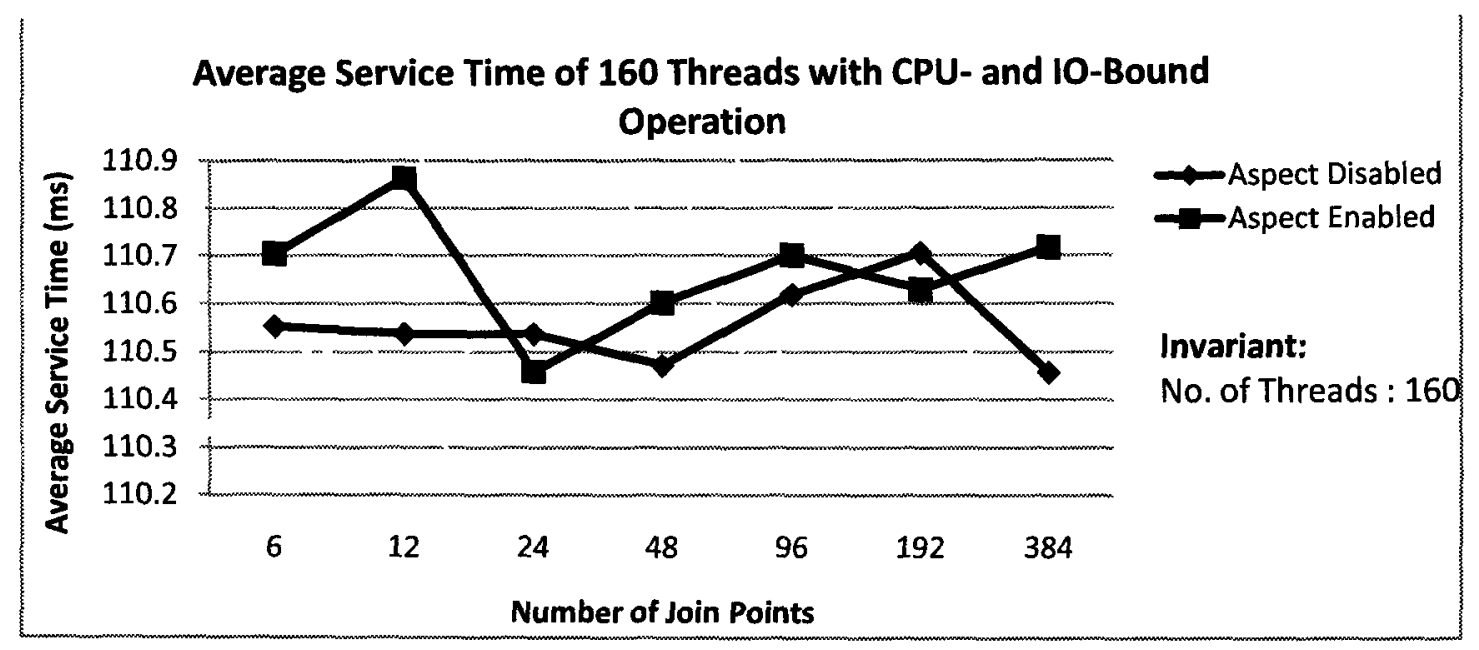

Figure 4.13 Average service time of a CPU- and IO-bound only operations for AOPenabled and AOP-disabled programs w.r.t. number of joint points 
Table 4.6 presents the cache fault data of Figure 4.13 above.

Table 4.6 Cache fault/second of LFs with different number of threads created under CPUand IO-bound operation

\begin{tabular}{|l|c|c|c|c|c|c|c|}
\hline No. Threads & 6 & 12 & 24 & 48 & 96 & 192 & 384 \\
\hline $\begin{array}{l}\text { Cache } \\
\text { fault/second } \\
\text { with Aspect } \\
\text { Disabled }\end{array}$ & 1069 & 1059 & 1846 & 1125 & 756 & 901.9 & 455.7 \\
\hline $\begin{array}{l}\text { Cache } \\
\text { fault/second } \\
\text { with Aspect } \\
\text { Enabled }\end{array}$ & 1570 & 1166 & 1316 & 1675 & 977.6 & 702.5 & 713.1 \\
\hline $\begin{array}{l}\text { Performance } \\
\text { Impact of } \\
\text { AOP } \\
\text { program (\%) }\end{array}$ & -0.13 & -0.29 & 0.071 & -0.11 & -0.07 & 0.068 & -0.23 \\
\hline
\end{tabular}

Figure 4.14 further illustrates the speed difference in percentage. Within the sample range of 6-384 of methods/join points, only 24 and 192 have positive values of performance impact. Even though this is not deterministic at all times, there is a chance of having an AOP-based program perform better in terms of average service time than the original base application software. There are seven measurements recorded. Out of these seven measurements, two were positive (e.g., AOP-enabled program is faster). Thus, there is about a $28 \%$ chance in this environment that an AOP-based program may yield better performance results. These are not exactly reproducible data, since we do not have control of the memory management. 


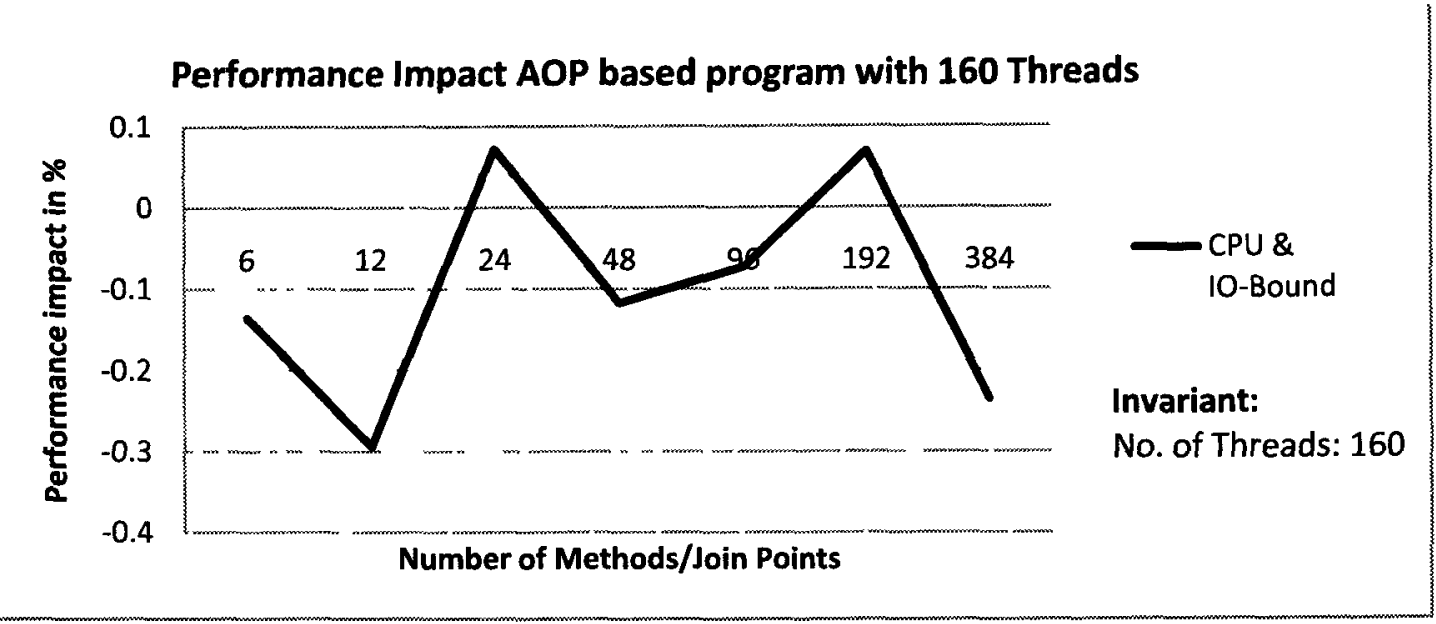

Figure 4.14 Performance of AOP-based program against the base application software

Figure 4.15 illustrates the system throughput with 160 threads allocated with both the I/O and CPU bound operation. They both run very closely to each other most of the time.

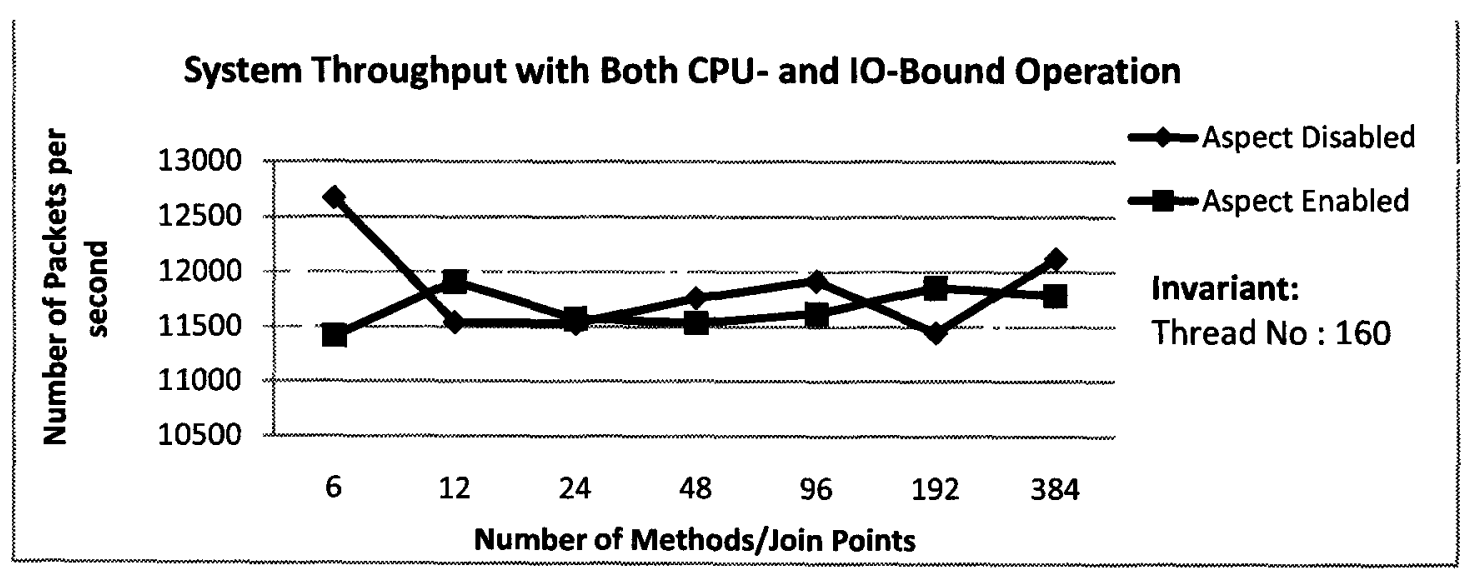

Figure 4.15 System throughput of LFs with 160 threads allocated 


\subsection{Performance Evaluation on HS/HA}

There are two intrinsic parameters in the HS/HA concurrency architecture; they are the size of the thread pool and the size of the message queue. The size of the thread pool determines the number of synchronous threads allocated to the system in the synchronous layer of HS/HA. In theory, setting the number of synchronous threads over a specific threshold, which depends on the hardware, will lead to high CPU and memory usage. CPU contentions and context switching of threads will slow down the overall performance of the system [Sch00][Zha10a]. Similarly, the size of the message queue could affect performance. If the size is too large, resources may be wasted and latency could be high. On the other hand, for very small queue size, some requests could be discarded.

The intention of the experiments presented in this section is to evaluate the effect of the HS/HA AOP-based program against the HS/HA non-AOP based program. The AOP-based program carries the exact same functionality of the original program while processing multiple runs of 32,000 requests. In order to identify the effect of the AOPenabled program, join points with seamless operations will also be manually added to the base code as well without altering the functionality of the original program. 


\subsubsection{Performance Evaluation on AOP Using HS/HA with Different Number of Threads in a CPU-Bound Operating Environment}

This section presents the result of changing the number of synchronous threads allocated in the thread pool of the HS/HA concurrency architecture. The invariants of the operating environment are set as follows:

- Message queue size: 20

- No emulated I/O operation

Figure 4.16 illustrates the average service time of the HS/HA concurrency architecture with respect to the number of threads. The average service time increased from 5 to 160 threads (and then dropped) as shown in Figure 4.16. Overall, the AOPenabled program still follows the trend of the original base program very closely. It can sometimes run faster and requires less average service time than the original non-AOP based program.

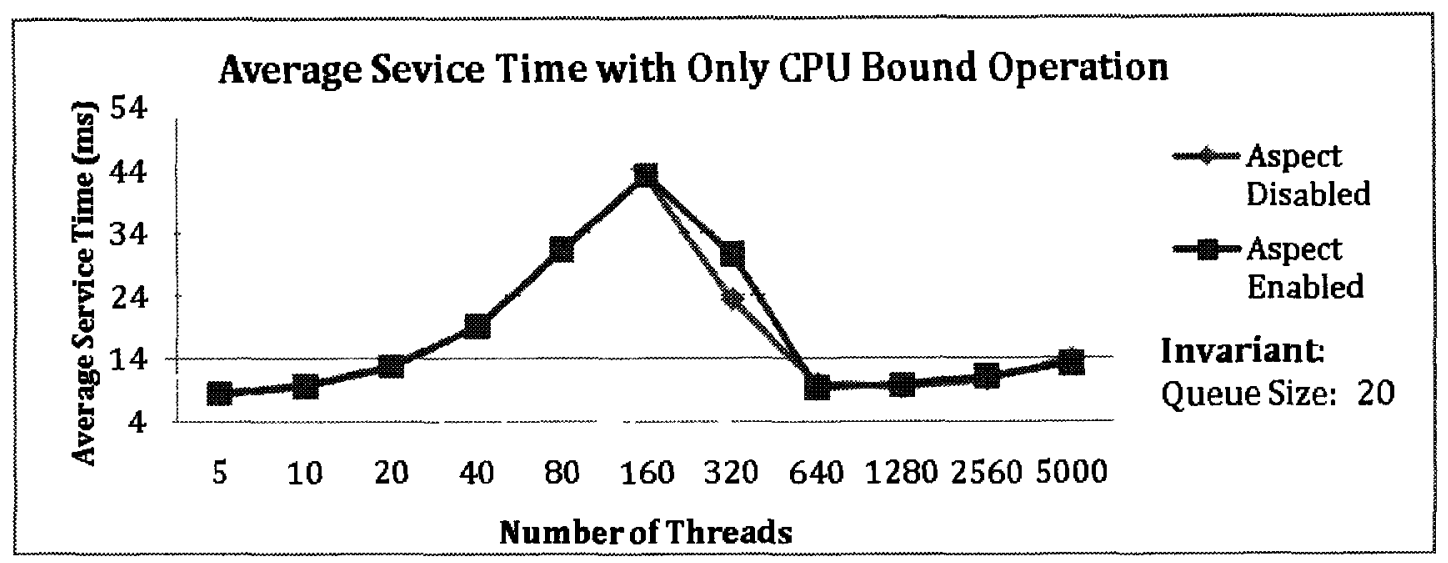

Figure 4.16 Average service time of HS/HA with only CPU-bound operation 
Apart from the case where the number of threads is 320 , the performance difference is not clear between AOP-enabled and AOP-disabled software. With the help of Table 4.7, which reveals the cache faults of each sample point, we can identify which program has better performance. Similar to the performance evaluation of LFs, memory management could contribute to the performance discrepancy. Since the non-AOP based program has lower cache faults than the AOP-based program at sample point 320 , it is therefore understandable that a non-AOP based program is faster.

Table 4.7 Cache fault/second of HS/HA with only CPU-bound operation

\begin{tabular}{|l|c|c|c|c|c|c|c|c|c|c|c|}
\hline No. Threads & 5 & 10 & 20 & 40 & 80 & 160 & 320 & 640 & 1280 & 2560 & 5000 \\
\hline $\begin{array}{l}\text { Cache } \\
\text { fault/second } \\
\text { with Aspect } \\
\text { Disabled }\end{array}$ & 4910 & 5535 & 3234 & 1400 & 1576 & 1349 & 1021 & 2389 & 1403 & 684 & 1361 \\
\hline $\begin{array}{l}\text { Cache } \\
\text { fault/second } \\
\text { with Aspect } \\
\text { Enabled }\end{array}$ & 4141 & 2869 & 2146 & 1473 & 1238 & 1869 & 1738 & 2137 & 1606 & 938 & 559 \\
\hline
\end{tabular}

Figure 4.17 illustrates the performance impact of the AOP-based program compared with the non-AOP software. The main point from this figure is that there is no absolute or obvious winner and the discrepancies in most cases are very small, except when the number of thread is 320 . 




Figure 4.17 Performance impact of AOP-based HS/HA with only CPU-bound operation

Figure 4.18 illustrates the system throughput of how the two programs perform in terms of how many packets they can process per second. It is not obvious to distinguish which one processes more requests when the number of threads allocated is from 5 to 2560. Then it becomes clear as the number of threads increases from 2560 to 5000 . The reason of not choosing 5120 as the number of thread is because the server machine cannot handle such high demands. Therefore, a smaller number, 5000, is chosen in this case. A very interesting point at sample point 320 is spotted. Even though the non-AOP based program at this point has lower average service time and lower cache faults, its throughput is almost the same as the AOP based program. As discussed in Section 4.4.3 for the LFs, the system throughput is also affected by three main factors that are not controlled by the general user-level application software. Those factors are the cache fault or memory management scheme, and Java virtual machine. 


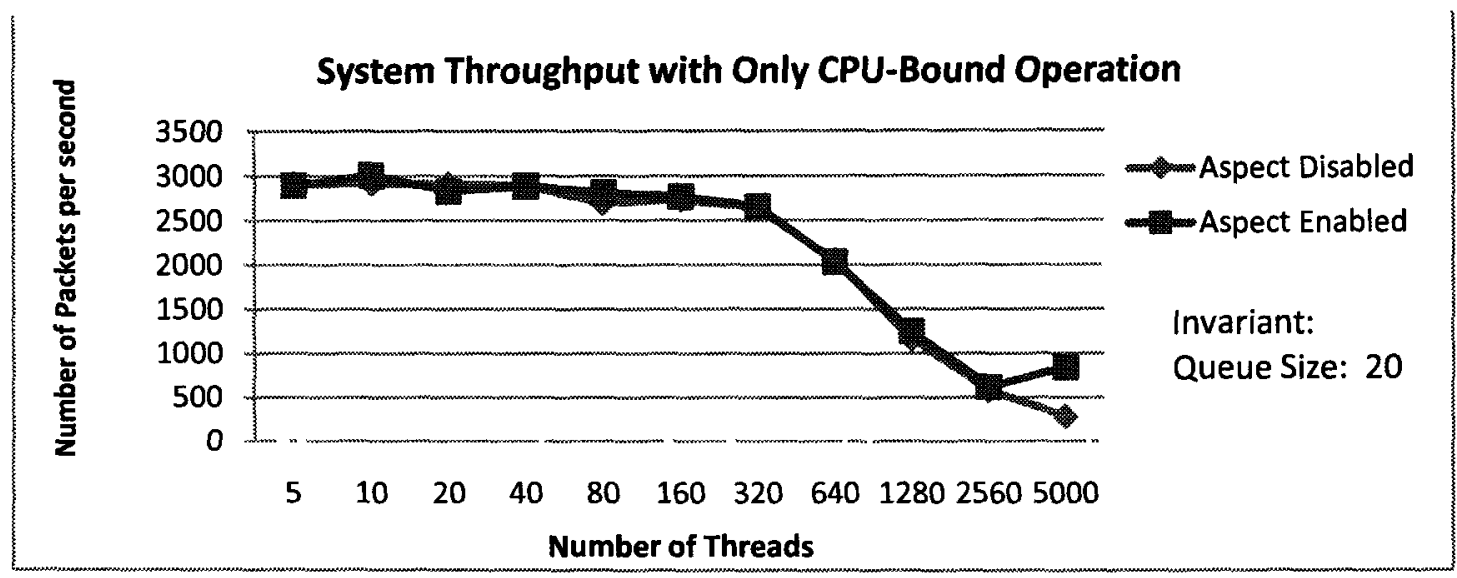

Figure 4.18 System throughputs of HS/HA with only CPU-bound operation

\subsubsection{Performance Evaluation on AOP Using HS/HA with Different Number of Threads in a CPU- and IO-Bound Operating Environment}

This section presents the performance result of having the HS/HA concurrency architecture set to the following invariants:

- Message queue size: 20

- IO-bound operation: $100 \mathrm{~ms}$

Figure 4.26 illustrates the average service time of the HS/HA concurrency architecture with an IO-bound operation that lasts for $100 \mathrm{~ms}$. It is not very obvious to see the difference between these two options. Both the AOP-based and non-AOP-based programs have very similar average service time as presented in Figure 4.19. 


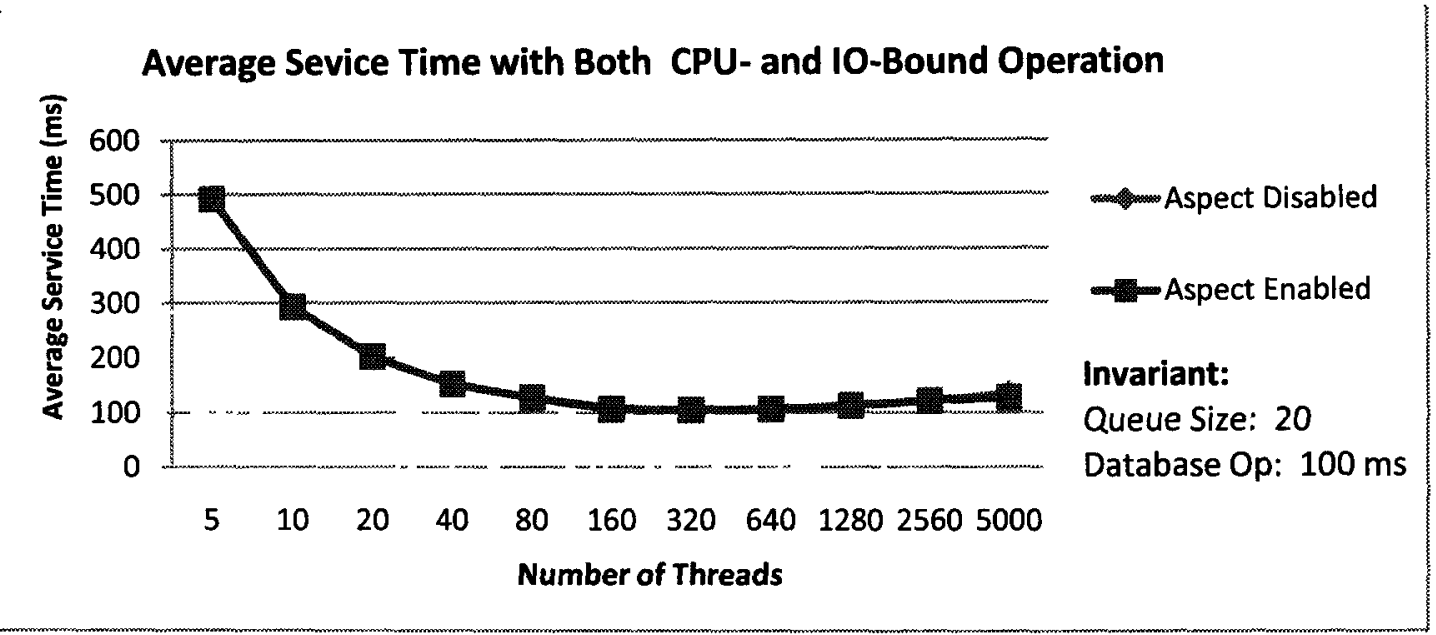

Figure 4.19 Average service time of HS/HA with CPU- and IO-bound operation

Table 4.8 presents the cache fault/second of both AOP and non-AOP based programs. The information in this table also indicates which program has better performance in average service time.

Table 4.8 Cache fault/second of HS/HA with CPU- and IO-bound operating environment

\begin{tabular}{|l|c|c|c|c|c|c|c|c|c|c|c|}
\hline No. Threads & 5 & 10 & 20 & 40 & 80 & 160 & 320 & 640 & 1280 & 2560 & 5000 \\
\hline $\begin{array}{l}\text { Cache } \\
\text { fault/second } \\
\text { with Aspect } \\
\text { Disabled }\end{array}$ & 356 & 306 & 245 & 395 & 475 & 367 & 375 & 586 & 377 & 435 & 676 \\
\hline $\begin{array}{l}\text { Cache } \\
\text { fault/second } \\
\text { with Aspect } \\
\text { Enabled }\end{array}$ & 322 & 303 & 247 & 404 & 486 & 356 & 629 & 735 & 366 & 684 & 489 \\
\hline
\end{tabular}

Figure 4.20 illustrates the performance impact of the AOP-based program compared to the base program. The results of sample points from 5 to 80 threads are quite 
steady. However, the AOP-based program runs faster over the base program at 160,1280 and 5000 threads.

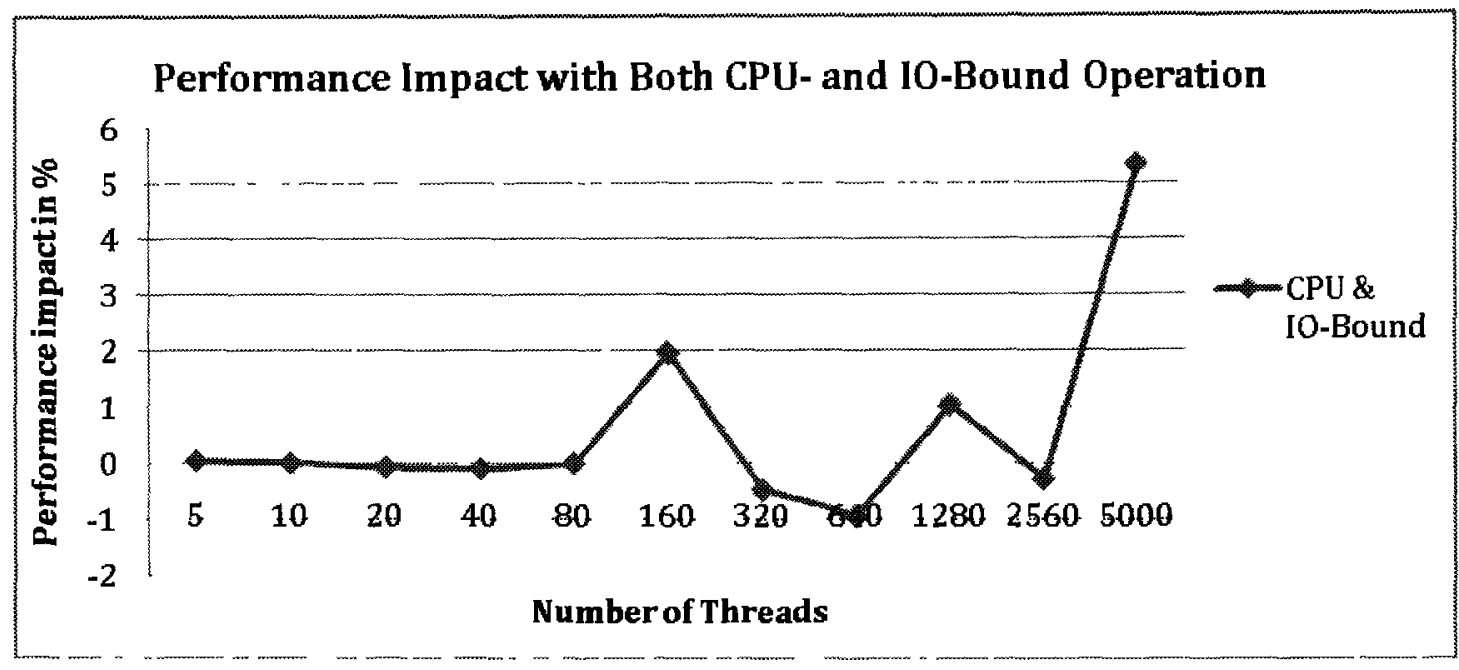

Figure 4.20 Performance impact of AOP-based HS/HA with CPU- and IO-bound operation

Figure 4.21 illustrates the system throughput of the HS/HA concurrency architecture. It is observed that they both process roughly the same amount of request per second from 5 to 160 . The AOP based program processes more requests per second than the base program from 320 to 5000 threads allocated to the thread pool. The result of having AOP program runs faster is depending on two factors, which are Java virtual machine and cache fault rate. Thus having less cache fault/second does not guarantee better system throughput.

An interesting sample point at 2560 from Figure 4.21 demonstrates that even when an AOP based program has disadvantage in cache fault rate, it still achieves better system throughput. However, the difference is not significant. 


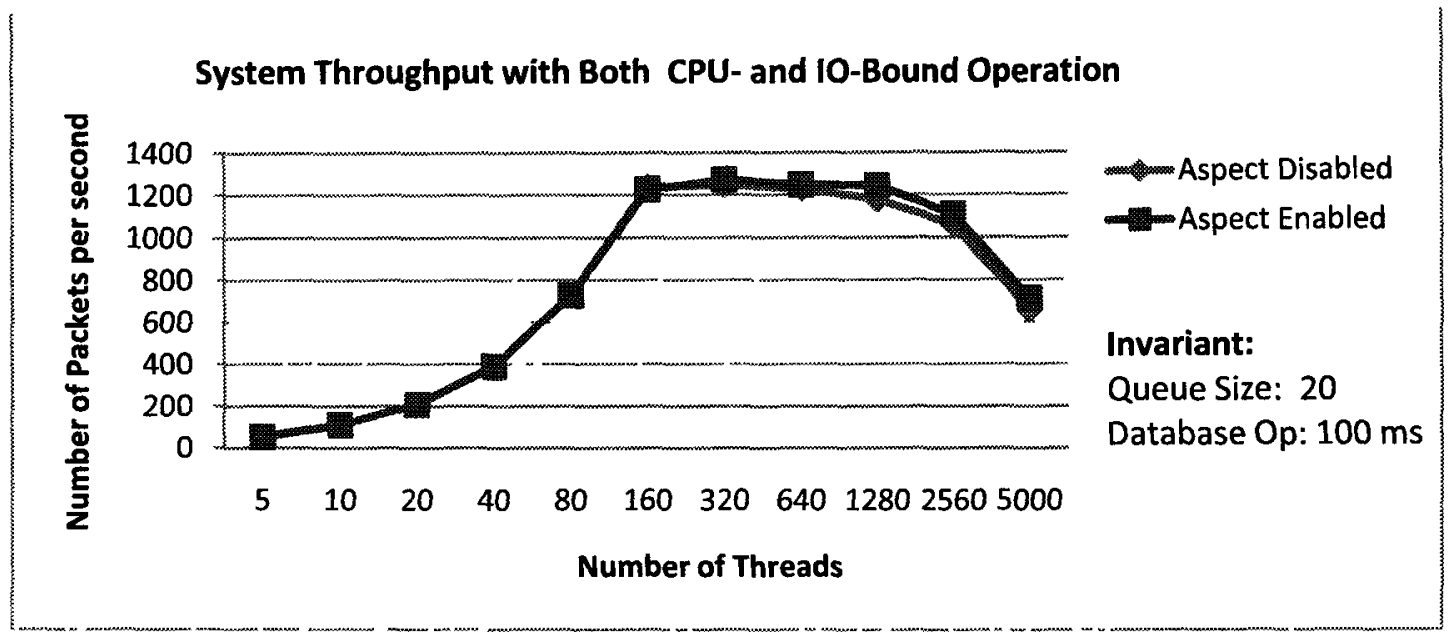

Figure 4.21 System throughput of HS/HA with CPU- and IO-bound operation

\subsubsection{Performance Evaluation of AOP Using HS/HA with Different Message Queue Sizes in a CPU-Bound Operating Environment}

This section presents the performance results of having the HS/HA concurrency architecture set to the following invariants:

- Number of synchronous thread: 160

- No IO-bound operation

Figure 4.22 illustrates the average service time of the HS/HA concurrency architecture with the message queue size increasing on the horizontal axis. The reason for choosing 160 as the number of synchronous threads allocated in the thread pool is explained in Section 4.4.3. The AOP-based program runs very closely with the base program. It is the same type of phenomenon observed in Sections 4.5.1 and 4.5.2, that AOP-based program has less average service time sometimes. The cache fault data of this experiment is presented in Table 4.9. 


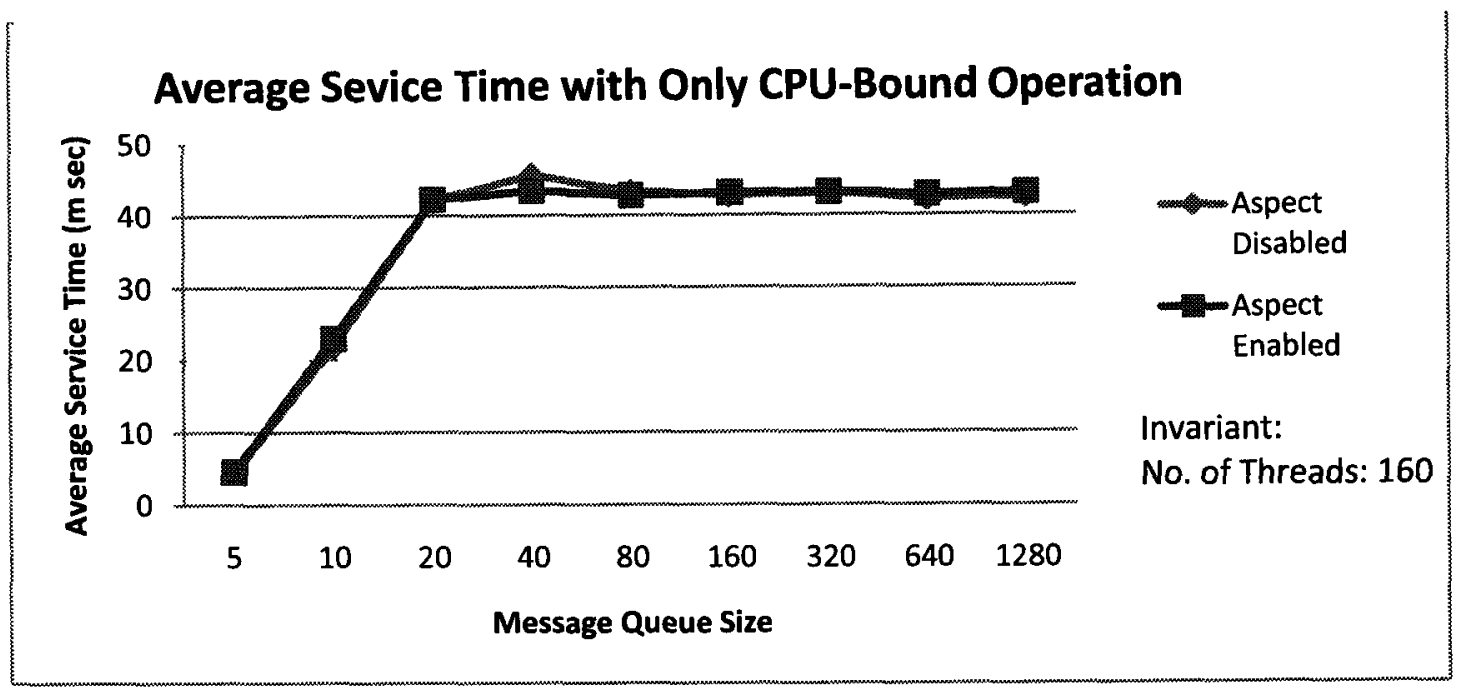

Figure 4.22 Average service time of AOP-enabled and AOP-disabled using HS/HA

\section{with only CPU-bound operations}

Table 4.9 presents the cache fault/second collected from Figure 4.22 . It provides a clue as to which sample point from Figure 4.22 has the AOP-based program running faster.

Table 4.9 Cache fault/second of HS/HA with only CPU operating environment

\begin{tabular}{|l|c|c|c|c|c|c|c|c|c|}
\hline $\begin{array}{l}\text { Message Queue } \\
\text { Size }\end{array}$ & 5 & 10 & 20 & 40 & 80 & 160 & 320 & 640 & 1280 \\
\hline $\begin{array}{l}\text { Cache } \\
\text { fault/second } \\
\text { with Aspect } \\
\text { Disabled }\end{array}$ & 528 & 579 & 513 & 2046 & 570 & 567 & 528 & 506 & 775 \\
\hline $\begin{array}{l}\text { Cache } \\
\text { fault/second } \\
\text { with Aspect } \\
\text { Enabled }\end{array}$ & 740 & 668 & 525 & 527 & 541 & 778 & 530 & 520 & 810 \\
\hline
\end{tabular}


Figure 4.23 presents the results of the performance impact of the AOP-based program. There are fluctuations at various points. If we look at sample point 40 in Figure 4.23 and Table 4.9, we notice that AOP-based program runs a lot faster than the nonAOP based program due to the fact that it has far less cache fault data than the base program. However, they both have very similar average service time overall.

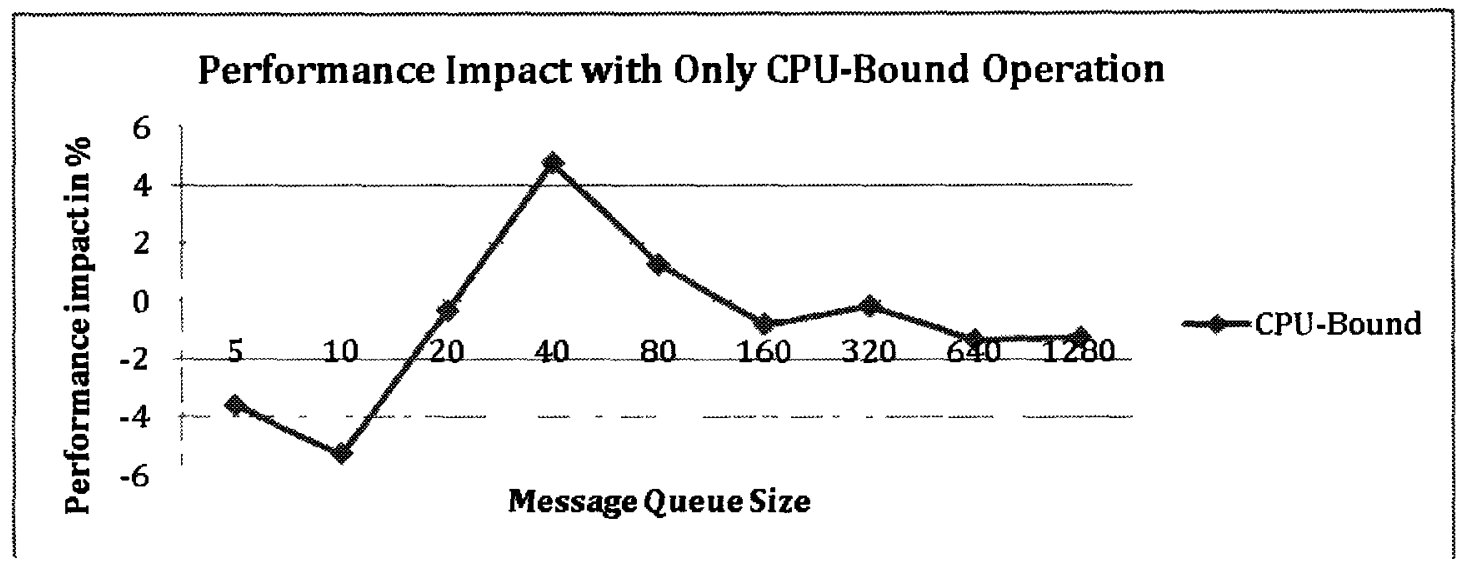

Figure 4.23 Performance impact of AOP-based HS/HA with only CPU-bound operation

Figure 4.24 illustrates the system throughput of both programs. The results of both system throughputs are similar but fluctuate a little as the size of message queue increases. 


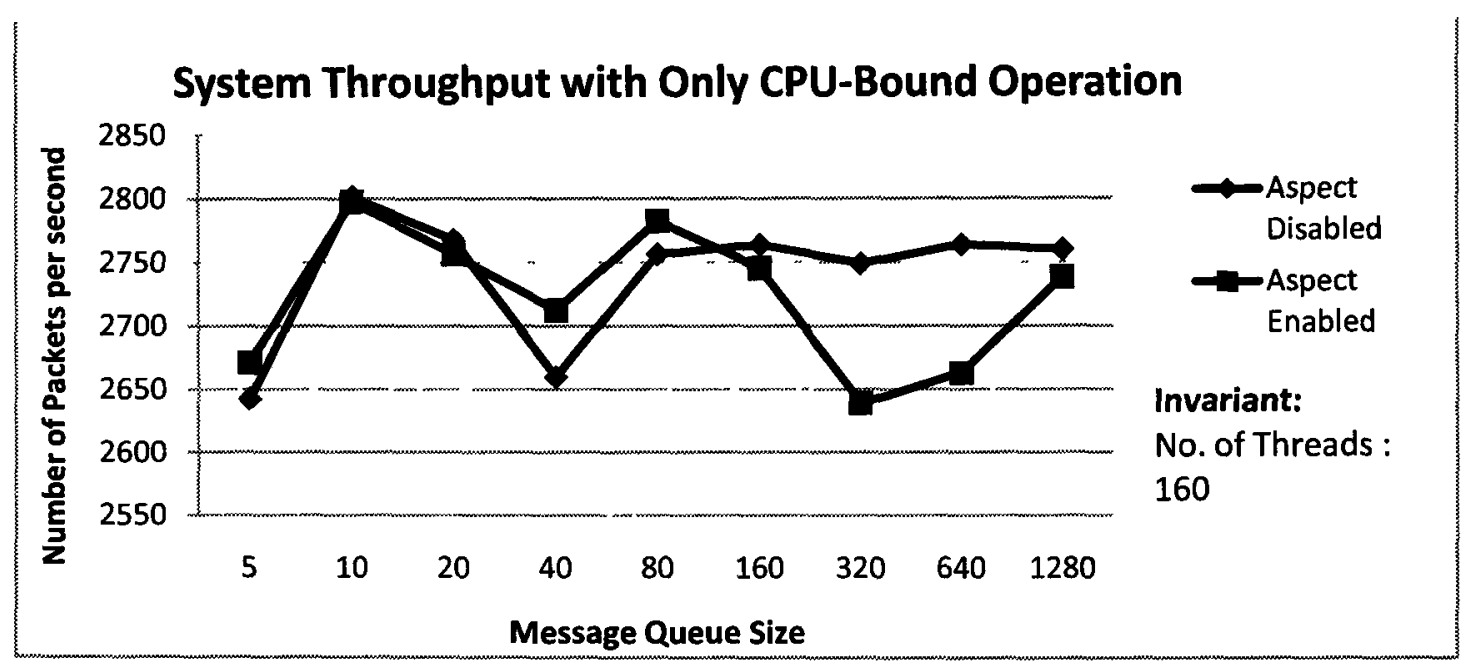

Figure 4.24 System throughput of HS/HA with only CPU bound operation

\subsubsection{Performance Evaluation of AOP Using HS/HA with Different Message Queue Size in a CPU- and IO-Bound Operating Environment}

This section is a further extension from Section 4.5.3 to verify how AOP paradigm behaves in an IO-bound operation. The invariants of this experiment setting are defined as follows:

- Number of synchronous thread: 160

- IO-bound operation: $100 \mathrm{~ms}$

Figure 4.25 presents the average service time of the HS/HA concurrency architecture with a different message queue size and a fixed IO-bound operation of 100 ms. There are some sample points in which the base program consumes less average service time than the AOP-based program (sample points 20, 160, 320 and 1280). Comparing this figure to Figure 4.25 , both design options show the same trend of 
stabilization when the queue size is greater than 40 . This indicates that the size of the message queue no longer needs to increase after 40 for this environment.

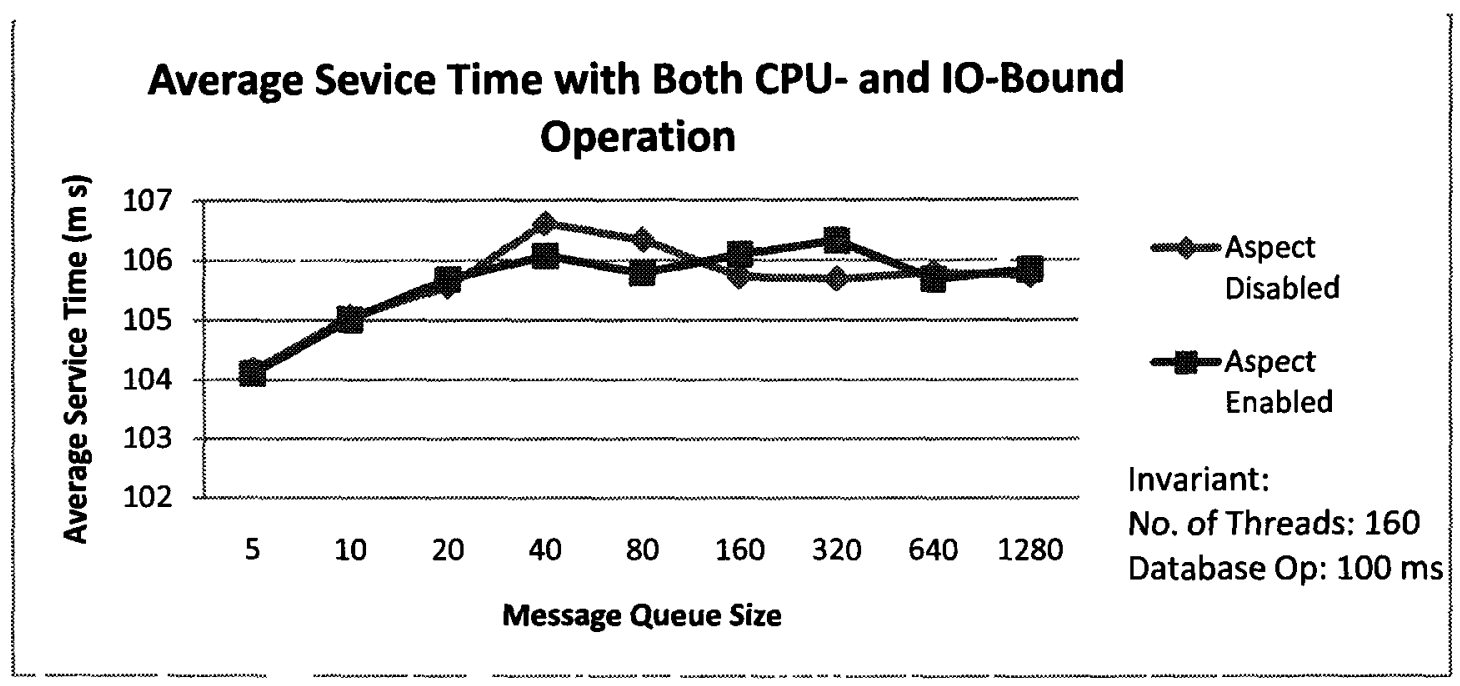

Figure 4.25 Average service time of HS/HA with CPU- and IO-Bound database operation

Table 4.10 presents the data of cache fault corresponding to Figure 4.25 . With the data, one can tell which program requires less average service time (Figure 4.25) if they both perform very similar to each other (e.g., message queue size at10). 
Table 4.10 Cache fault/second of HS/HA with CPU- and IO-Bound operating environment

\begin{tabular}{|l|c|c|c|c|c|c|c|c|c|}
\hline $\begin{array}{l}\text { Message Queue } \\
\text { Size }\end{array}$ & 5 & 10 & 20 & 40 & 80 & 160 & 320 & 640 & 1280 \\
\hline $\begin{array}{l}\text { Cache } \\
\text { fault/second } \\
\text { with Aspect } \\
\text { Disabled }\end{array}$ & 505 & 374 & 379 & 365 & 488 & 359 & 329 & 351 & 380 \\
\hline $\begin{array}{l}\text { Cache } \\
\text { fault/second } \\
\text { with Aspect } \\
\text { Enabled }\end{array}$ & 361 & 368 & 382 & 348 & 336 & 693 & 583 & 345 & 590 \\
\hline
\end{tabular}

Figure 4.26 illustrates the performance impact of the AOP-based program. The result of the performance impact does conform to Table 4.10. From the cache fault data, we can identify when the AOP-based program runs faster than the base program in terms of their average service time.



Figure 4.26 Performance impact of AOP-based HS/HA with CPU- and IO-bound operation 
Figure 4.27 illustrates the system throughput of both programs. Out of nine sample points taken, four results demonstrate that the AOP based program processed more packets per second than the non-AOP base program.



Figure 4.27 System throughput of HS/HA with CPU- and IO-bound operation

\subsubsection{Performance Evaluation of HS/HA with Different Number of Join Points in a CPU-Bound Operating Environment}

This section is similar to Section 4.4.3. In order to verify the hypothesis stated earlier in Section 4.1, various numbers of join points are manually added into the base program without altering the original functionalities of the software. The invariants of the experiment setting are defined as follows:

- Number of synchronous thread: 40

- Message queue size: 40

- No IO-bound operation 
In theory, when the number of join points increases, the control flow of the system will have to switch back and forth frequently from the base code to the aspect code. The overhead should lead to an increase of average service time. Since we have collected quite a lot of data in the previous sections for LFs, it is also expected that such theoretical results may not be consistent with the expectation.

Figure 4.28 presents the results of average service time with respect to different numbers of join points. The overall average service time of the AOP-based program is more than the base program. It is observed from Figure 4.28 that the base program generally needs less average service time than the AOP program. This confirms the hypothesis that the the higher the number of join points, the more overhead is required.



Figure 4.28 Average service time of a CPU-bound only operations for AOP-enabled and AOP-disabled programs w.r.t. number of joint points 
Table 4.11 presents the cache faults corresponding to Figure 4.28. Apart from the sample point 96 , one can clearly identify which program requires less average service time.

Table 4.11 Cache fault/second of HS/HA with CPU-bound operating environment

\begin{tabular}{|l|c|c|c|c|c|c|c|}
\hline $\begin{array}{l}\text { No. Methods/ Join } \\
\text { Points }\end{array}$ & 6 & 12 & 24 & 48 & 96 & 192 & 384 \\
\hline $\begin{array}{l}\text { Cache fault/second } \\
\text { with Aspect } \\
\text { Disabled }\end{array}$ & 561 & 563 & 590 & 608 & 610 & 635 & 812 \\
\hline $\begin{array}{l}\text { Cache fault/second } \\
\text { with Aspect } \\
\text { Enabled }\end{array}$ & 600 & 606 & 785 & 611 & 584 & 701 & 827 \\
\hline
\end{tabular}

Figure 4.29 illustrates the performance impact of the AOP-based program. It shows that the base program runs faster most of the time, except at sample point 96 . If we cross check with Table 4.11, we can understand why it has a positive value. It is because the AOP-based program has less cache faults for different join points.

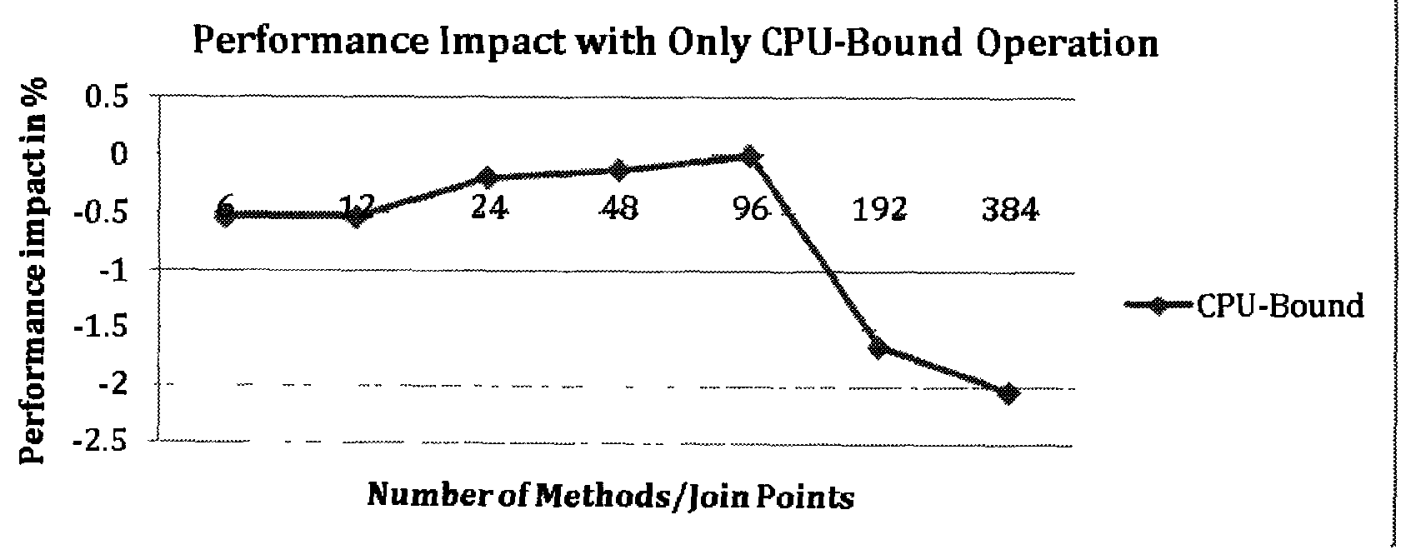

Figure 4.29 Performance impact of HS/HA AOP-based program 
Figure 4.30 illustrates how the AOP based program behaves in term of system throughput compared to the base program. Overall, the discrepancy between AOP based program and the base program is very small in most of the cases. When the number of joint point is 12 , the difference is obvious. But there are factors related to the memory management or Java virtual machine that is not under the control of our application programs.

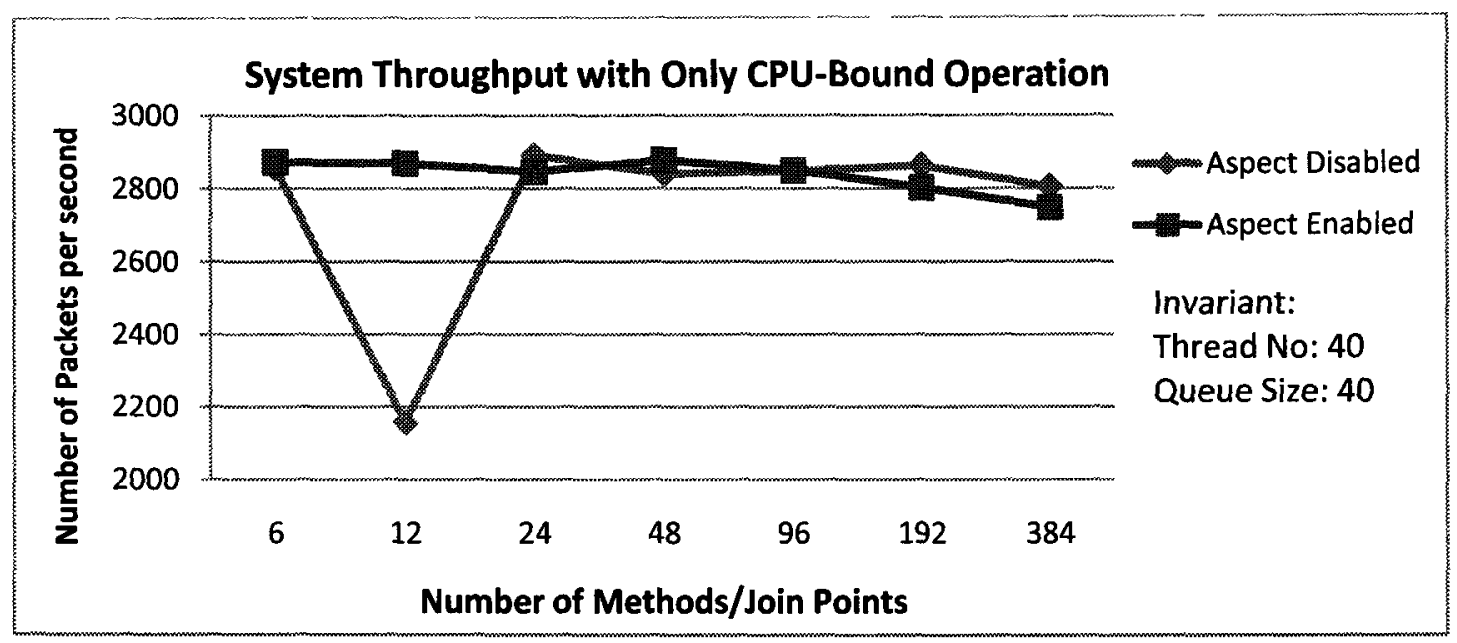

Figure 4.30 System throughputs of HS/HA with CPU-bound operation only

\subsubsection{Performance Evaluation of AOP Using HS/HA with Different Number of Join Points in a CPU- and IO-Bound Operating Environment}

The invariants of this experiment setting are as follows:

- Number of synchronous thread: 40

- Message queue size: 40

- IO-bound operation: $100 \mathrm{~ms}$ 
Section 4.5.5 has demonstrated the effect of AOP-based program with the number of join points as a variable in a CPU-bound operational environment. Thus, this section verifies the performance impact of the AOP-based program in a CPU- and IO-bound operating environment with an IO-bound operation of $100 \mathrm{~ms}$.

Figure 4.31 presents the average service time of both the AOP-based and base program. The AOP-based program runs very closely to the base program, as the scale of $\mathrm{Y}$-axis is very small.

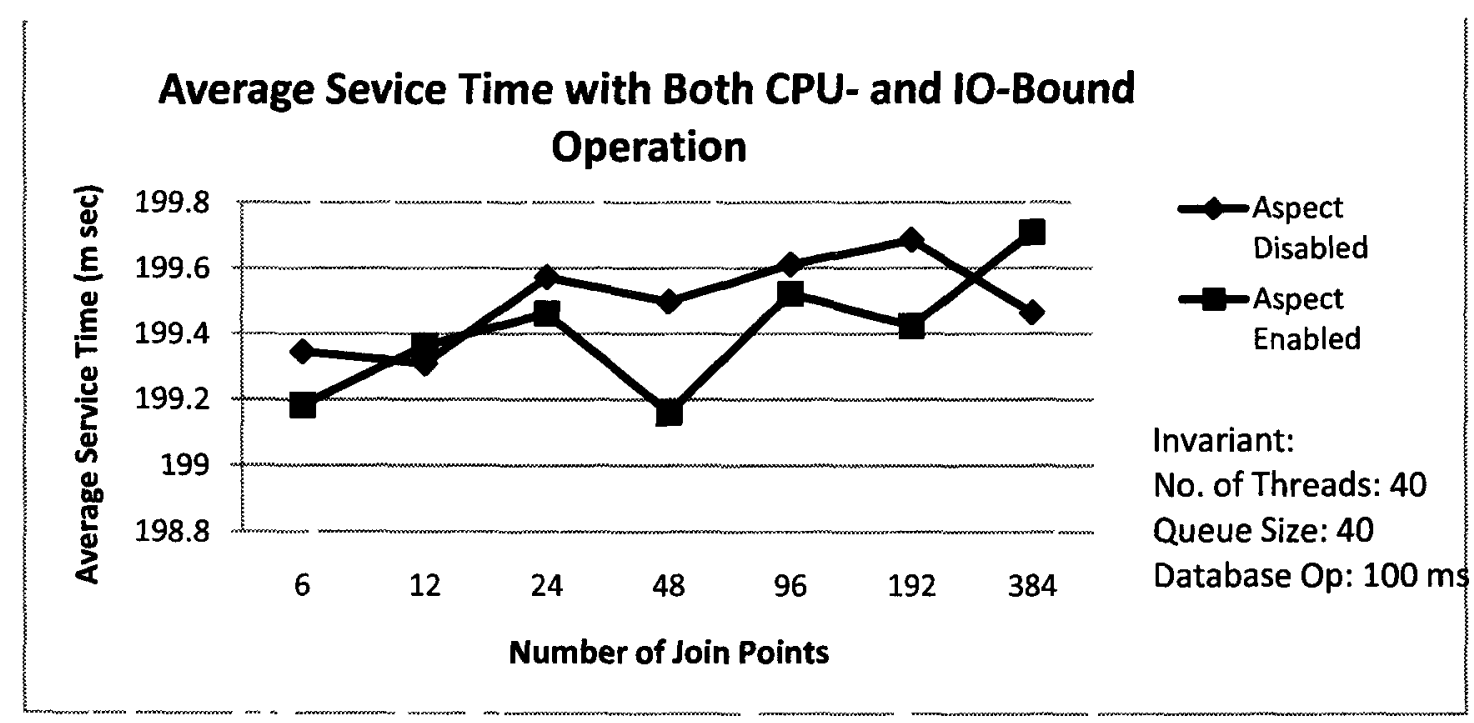

Figure 4.31 Average service time of a CPU- and IO-Bound operations for AOPenabled and AOP-disabled programs w.r.t. number of joint points

Table 4.12 presents the cache fault data for the experiment. It shows which program runs faster than the other from the figure or we can also cross check their cache fault data as shown in Table 4.12. 
Table 4.12 Cache fault/second of HS/HA with CPU- and IO-Bound operating environment

\begin{tabular}{|l|c|c|c|c|c|c|c|}
\hline $\begin{array}{l}\text { No. Methods/ Join } \\
\text { Points }\end{array}$ & 6 & 12 & 24 & 48 & 96 & 192 & 384 \\
\hline $\begin{array}{l}\text { Cache fault/second } \\
\text { with Aspect } \\
\text { Disabled }\end{array}$ & 302 & 311 & 297 & 302 & 294 & 300 & 335 \\
\hline $\begin{array}{l}\text { Cache fault/second } \\
\text { with Aspect } \\
\text { Enabled }\end{array}$ & 278 & 630 & 266 & 284 & 276 & 296 & 677 \\
\hline
\end{tabular}

Figure 4.32 illustrates the performance impact of the AOP-based program with CPU- and IO-bound operation. By looking at the results from Figure 4.32, AOP paradigm does not have much impact in this case.



Figure 4.32 Performance impact of HS/HA with CPU- and IO-bound operation

Figure 4.33 presents the system throughput of AOP and base program. The difference between these two software systems is negligible in this scenario. 




Figure 4.33 System throughput of HS/HA with CPU- and IO-bound operation

\subsection{Summary}

This chapter describes the result of the experimental process undertaken to compare the performance impact of AOP paradigm on system software. Two concurrency architectural patterns are chosen, HS/HA and LFs, due to their popularity. Both patterns were modified to incorporate aspects. The aspects embedded programs have the same functionalities as the base programs.

The summaries presented from Figure 4.34 to Figure 4.37 show that the AOPbased programs follow the performance trend of the original base program closely, and they sometimes even run faster than the non-AOP based counterparts. 


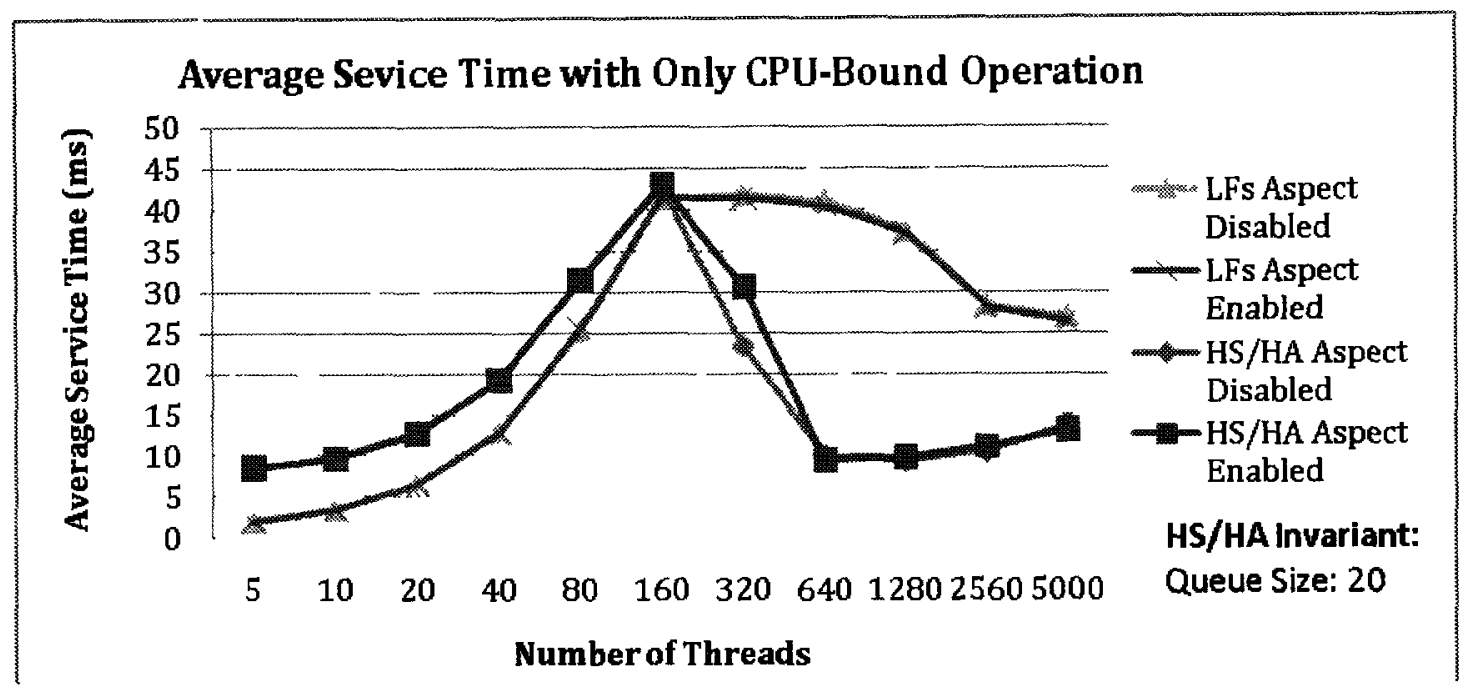

Figure 4.34 Average service time of LFs and HS/Ha with CPU-bound only operations

Figure 4.35 illustrates the performance impact of AOP for LFs and HS/HA concurrency architectures. There is an outlier at sample point 320 . However, if we look at Figure 4.34, the overall average service time of HS/HA is very similar in both the AOP and the base program.

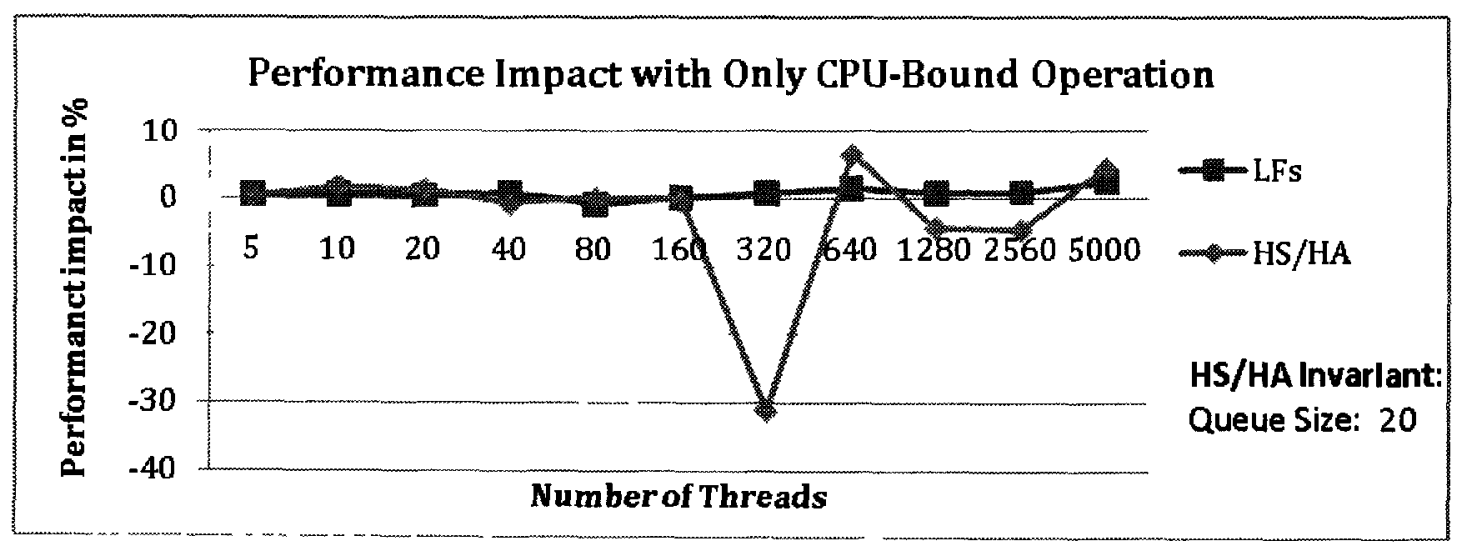

Figure 4.35 Performance impact of LFs and HS/HA with CPU-bound operation 
Figure 4.36 illustrates average service time of LFs and HS/HA in a CPU- and IObound operation environment. It is observed that the AOP-based programs run closely to their original base programs respectively.

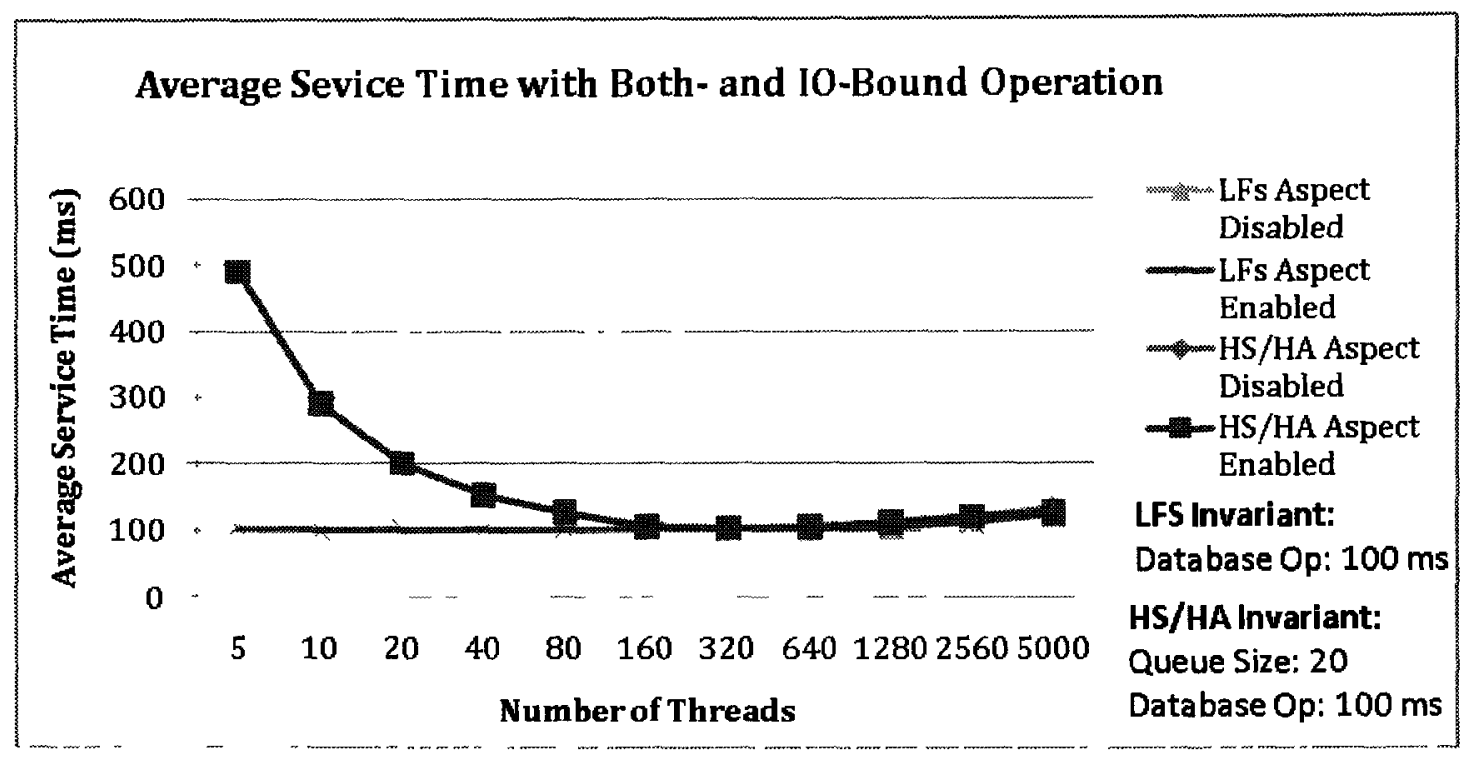

Figure 4.36 Average service time of LFs and HS/HA with CPU- and IO-bound operations

Figure 4.37 illustrates the performance impact of LFs and HS/HA concurrency farchitectures. It has been observed that the performance impact of AOP is negligible or AOP becomes even slightly better than the base program (negative values). Also, both LFs and HS/HA have fluctuations when the number of threads is high. 


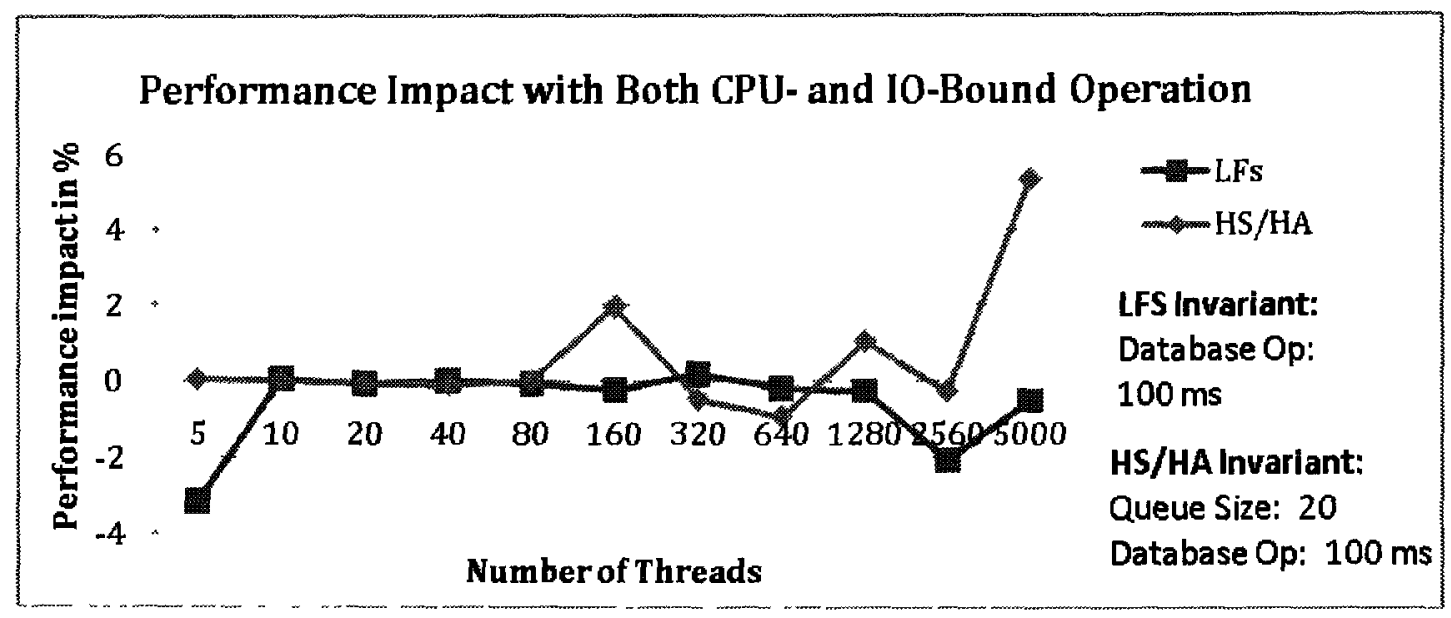

Figure 4.37 Performance impact of LFs and HS/HA with CPU- and IO-bound operation 


\section{Chapter 5 Conclusion and Future Work}

This chapter concludes the work presented in this thesis and presents some potential future research topics.

\subsection{Conclusion}

In order to separate the present concerns and anticipated future changes, aspectoriented programming can effectively help. However, performance has been a concern in practice for AOP. The thesis first applied the aspect-oriented design and programming technologies to software programs that used two architectural patterns, LFs and HS/HA, in concurrent programming. Concurrent programming involves synchronization of multiple components and could be very complex. Using AOD/AOP could simplify the design by separating the concurrency aspect. To date, there is no research reported on applying AOD/AOP to LFs or HS/HA [Aok07][Ape08a][Ape08b][Asp10][Kim08a] [Kim08b][Kle07][Lea10].

Further, a number of experiments have been conducted in this research to evaluate the performance impact of AOP. Based on the results we have collected, it appears that the performance overhead of AOP is negligible. In some cases, based on our results, AOP-enabled programs even outperformed their counterparts.

The hypotheses presented in Chapter 4 are:

1. The performance of the base code is somewhat degraded when AOP approach is applied to the base application software. 
2. Incremental effect on performance becomes more obvious as the number of join points increases.

Based on the analysis and results, we can conclude Hypotheses 1 as follows:

- The introduction of AOP paradigm does not have significant effect on the performance of the base program

- Oftentimes, the AOP programs do have equal or better performance vis-àvis the base programs. This could be due to the operating system cache fault rate. Since we do not have control over the memory management, we cannot generalize the effect.

Hypothesis 2: We may reject this hypothesis based on the following facts:

- The results of our experiment show that the introduction of huge number of joint points does not have significant effect on the performance. Where there are differences, it is very close to the extent that one can ignore such differences.

In conclusion, the analysis and the results obtained in chapter 4 provide us with the idea that the AOP overhead, caused by switching of control flow, is small and negligible in both of the concurrency architecture. In general, people think that the overhead in using AOP paradigm is very high and that the performance impact is huge. However, in this thesis, we find out that the performance of AOP-based program is as good as non-AOP based program. 


\subsection{Future Work}

There are various areas that could be further investigated:

- The effect of memory management on performance can be studied. Some factors to consider include the cache size, main memory size, and even the program size.

- The performance impact of programs with a high degree of code scattering or code tangling is left to find out.

- Evaluate the performance of AOP in embedded devices or mobile phones, like Android phones. Since the Android is an OS based on Java, AspectJ can possibly be implemented on mobile devices powered by Android. 


\section{References}

[Aik98] P. Aiken, Reverse Engineering of Data, IBM Systems Journal 37(2), 1998.

[Aok07] Y. Aoki, and S. Chiba, "Performance improvement for persistent systems by AOP", Proc. of the Software-Engineering Properties of Languages and Aspect Technologies (SPLAT),2007.

[Ape08a] S. Apel, C. Kästner, and D. Batory. "Program Refactoring using Functional Aspects," Proc. of the $7^{\text {th }}$ International Conference on Generative Programming and Component Engineering, 2008.

[Ape08b] S. Apel and D. Batory, "How AspectJ is Used: An Analysis of Eleven AspectJ Programs", Technical report MIP-0801, Universität Passau, Passau, Germany, 2008.

[Asp10] "AspectJ: Crosscutting Objects for Better Modularity," Eclipse.org, [Online.] Available: http://www.eclipse.org/aspecti/index.php. [Accessed: Jun. 10, 2010].

[Bla10] "Design Patterns: Layers,"'Online] Available: http://stevenblack.com/PTNLayers.html. [Access: Jul. 16, 2010].

[Byr91] E. J. Byrne, "Software reverse engineering: A case study", Proc. of the Software Practice and Experience, 1991, pp. 1349-1364.

[Cha03] F. G. Chavez, and C. P. Lucena, "A Theory of Aspects for Aspect-oriented Software Development," Proc. of the Brazilian Symposium on Software Engineering, 2003.

[Chi90] E.J Chikofsky, and J.H. Cross II, "Reverse Engineering and Design Recovery: A Taxonomy in IEEE Software", IEEE Computer Society, pp. 13-17, 1990.

[Cla99] Siobhan Clarket ,"Extending UML Metamodel for Design Composition", Proc. of the $3^{\text {rd }}$ European Conference on Object-Oriented Programming (ECOOP), 1999.

[Cun06] C. A. Cunha, J. L. Sobral, Monteiro, and M.P. Monteiro, "Reusable AspectOriented Implementations of Concurrency Control Patterns and Mechanisms," Proc. of the Aspect-Oriented Software Development, 2006, pp. 134 - 145.

[CPU10] CPUID Technical Resources, "What is CPU-Z", 
http://www.cpuid.com/softwares/cpu-z.html, last accessed on Sep. 28, 2010.

[Dat10] "Software Design Patterns" Data \& Object Factory, [Online]. Available: http://www.dofactory.com/Patterns/Patterns.aspx. [Accessed: May 31, 2010].

[Dem03] S. Demeyer, S. Ducasse, and O. Nierstrasz, Object-Oriented Reengineering Patterns, San Francisco, Morgan Kaufmann, 2003.

[Goe06] B. Goetz, T. Peierls, J. Bloch, J. Bowbeer, D. Holmes, and D. Lea, Java Concurrency in Practice, Addison Wesley Professional, May 2006.

[Gra03] J. Gradecki, and N. Lesiecki, Mastering AspectJ, Wiley Publishing, 2003.

[Har05] E. Harold, Java network Programming, O’Reilly, Sebastopol, CA, USA, 2005.

[Lad03] R. Laddad, AspectJ in Action: Practical Aspect-Oriented Programming, Manning, Greenwich, 2003.

[Lea99] D. Lea, Concurrent Programming in Java: Design Principles and Patterns, Addison-Wesley, Boston, MA, USA, 1999.

[Lea10] G. T. Leavens, "Introduction to Aspect-Oriented Programming and its Reasoning Problems", June 2010. [Online]. Available: http://research.microsoft.com/enus/um/people/leino/ifip-wg2.3/media/48/leavens-concerningreasoning.ppsx. [Accessed: Jun 8, 2010].

[Lun06] C. H. Lung, K. Selvarajah, B. Balasubramaniam, P. Elankeswaran, and U. Gopalasundaram "Architecture-Centric Software Generation: An Experimental Study on Distributed Systems", Proc. of the $5^{\text {th }}$ International Conference on Generative Programming and Component Engineering, 2006.

[Mil05] R. Miles, AspectJ Cookbook, O’REILLY, 2005.

[Ms06] Microsoft Support, "How to manage System Monitor counters in Windows XP", Microsoft, June, 2006, http://support.microsoft.com/kb/305610, last accessed on Jun. $30^{\text {th }}, 2010$.

[Ker05] M. Kersten, “AOP@Work: AOP tools comparision, Part1,” IBM, Feb. 8, 2005, [Online.] Available: http://www.ibm.com/developerworks/java/library/jaopwork1/. [Accessed: Jun. 10, 2010].

[Kic97] G. Kiczales, “Aspect-Oriented Programming”, Proc of the European Conference 
on Object-Oriented Programming (ECOOP), Finland, 1997.

[Kim08a] C. H. P. Kim, K. Czarnecki, and D. Batory, "On-Demand Materialization of Aspects for Application Development," Proc. of the Software-Engineering Properties of Languages and Aspect Technologies (SPLAT), 2008, pp. 1-6.

[Kim08b] C. Kim, C. Kästner, and D. Batory, "On the Modularity of Feature Interactions," Prof. of the International Conference on Generative Programming and Component Engineering (GPCE), 2008, pp. 23-34.

[Kle07] M. Kleyman, S. Tyszberowicz and A.Yehudai, "Refactoring Aspects into Java Code," IEEE, 2007. [Online]. Available:

http://ieeexplore.ieee.org/stamp/stamp.jsp?arnumber $=04384083$. [Accessed: Apr. 24, 2010].

[Oak04] A. Oaks and H. Wong, Java Threads, $3^{\text {rd }}$ ed., O'Reilly, Sebastopol, CA, USA, 2004.

[OMG07] OMG White Paper on the Profile mechanism V1.0. OMG Document ad/9904-07.

[Oso07] T. Osogami and S. Kato S, "Optimizing System Configurations Quickly by Guessing at the Performance", Proc. of 2007 ACM Special Interest Group on Measurement and Evaluation, June 07, pp.145-156.

[Rug93] S. Rugaber and R. Clayton, "The representation problem in reverse engineering", Proc. of the Working Conference on Reverse Engineering, 1993, pp. 8-16.

[Sch96] D. C. Schmidt and C. D. Cranor, "Half-Sync/Half-Async: an Architectural Pattern for Efficient and Well-structured Concurrent I/O," in Pattern Languages of Program Design, Reading, MA: Addison-Wesley, 1996.

[Sch00] D. C. Schmidt, M. Stal, H. Rohnert and F. Bushmann, Pattern-Oriented Software Architecture: Patterns for Concurrent and Networked Objects, John Wiley \& Sons, 2000.

[Shi00] J. Shirazi, Java Performance Tuning, O'Reilly, Sebastopol, CA, USA, 2000.

[Spi10] "Chapter 6: Aspect Oriented Programming with Spring," [Online.] Available: http://static.springsource.org/spring/docs/2.0.x/reference/aop.html. [Accessed: Jun. 10, 2010]. 
[Sor07] S. Soares, P. Borba, "Towards Reusable and Modular Aspect-Oriented Concurrency Control," Prof. of the ACM Symposium on Applied computing, 2007, pp. $1293-1294$.

[Sub05] S. Subotic, and J. Bishop, "Emergent Behaviour of Aspects in High Performance and Distributed Computing". Proc. of the South African Institute for Computer Scientists and Information Technologists (SAICSIT), 2005, pp. 11-19.

[UML97a] UML Summery V1.1, OMG, ad/97-08-03, www.rational.com/UML.

[UML97b] UML Syntax and Semantics Guide V1.1, OMG, ad/97-08-03, www.rational.com/UML.

[War92] R. Warden, Software Reuse and Reverse Engineering in Practice, Chapman \& Hall, 1992. pp. 283-305.

[Wel00] M. Welsh , S. Gribble , E. Brewer, and D. Culler, "A Design Framework for Highly Concurrent Systems," Tech. Rep., UC Berkeley, April 2000

[Wik10a] Wikipedia, "Reverse Engineering", [Online]. Available: http://en.wikipedia.org/wiki/Reverse engineering [Accessed: May. 24, 2010].

[Wik 10b] "Aspect-Oriented Programming”, Wikpedia, [Online.] Available: http://en.wikipedia.org/wiki/Aspect-oriented programming. [Accessed: Jun. 10, 2010].

[Zab98] F. Zabatta and K. Ying, "Dynamic thread creation: an asynchronous load balancing scheme for parallel searches", Proc. of $10^{\text {th }}$ International Conference on Parallel and Distributed Computing and Systems, 1998.

[Zha08] Y. Zhang, Y. Chen, J. Zhang, and Q. Wang, "Resolving Synchronization and Analyzing Based on Aspect-Oriented Programming," Proc. of the International Symposium on Computer Science and Computational Technology (ISCSCT), vol. 1, pp.34-37, 2008.

[Zha09] J. Zhang, Y. Chen, G. Liu, and H Li, "An Aspectual State Model and its Realization based on AOP," Proc. of the 2009 WRI World Congress on Software Engineering, vol.3, pp. 163-166.

[Zha10a] X. Zhang, An Architecture-based Self-Adaptive Framework for Performance and Reliability Improvement, M.S. thesis, Carleton University, Ottawa, ON, Canada, 2010. 
[Zha10b] Y. Zhang, J. Zhang, and D. Zhang, "Implementing and Testing ProducerConsumer Problem Using Aspect-Oriented Programming," Proc. of the $5^{\text {th }}$ International Conference on Information Assurance and Security, 2009, pp.749-752. 


\section{Appendix A Java and AspectJ code for LFs}



The following code is the LFsThreadPool class being advised by LeaderProcess aspect LFs: public class LFsThreadPool i

private int number of worker thread;

private ArrayList<IFsThread> worker_Thread_Pool;

private IoService ioservice;

private int numpackage, count;

private boolean needTostop = false;

public ConcurrenthashMap<Integer, ServiceTime> hashMap; public long firstPacketArivalTime;

private LoggingService logging;

private Authenticationservice authenticate;

private Authorizationservice authorize;

public LFsThreadPool (int numPackage)

\{

worker_Thread_Pool = new ArrayList $\langle$ LFsThread $>()$; ioservice = new IOService(); 


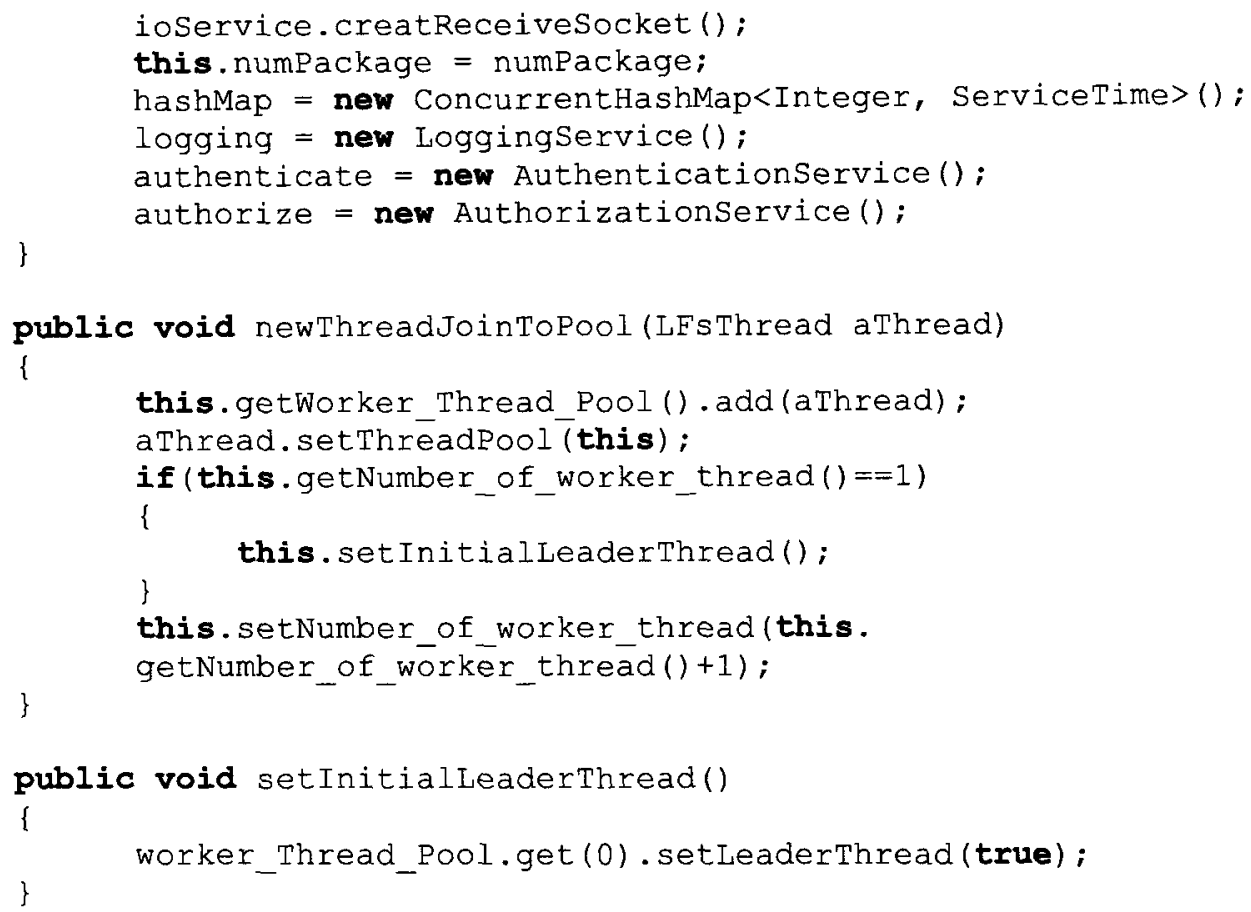




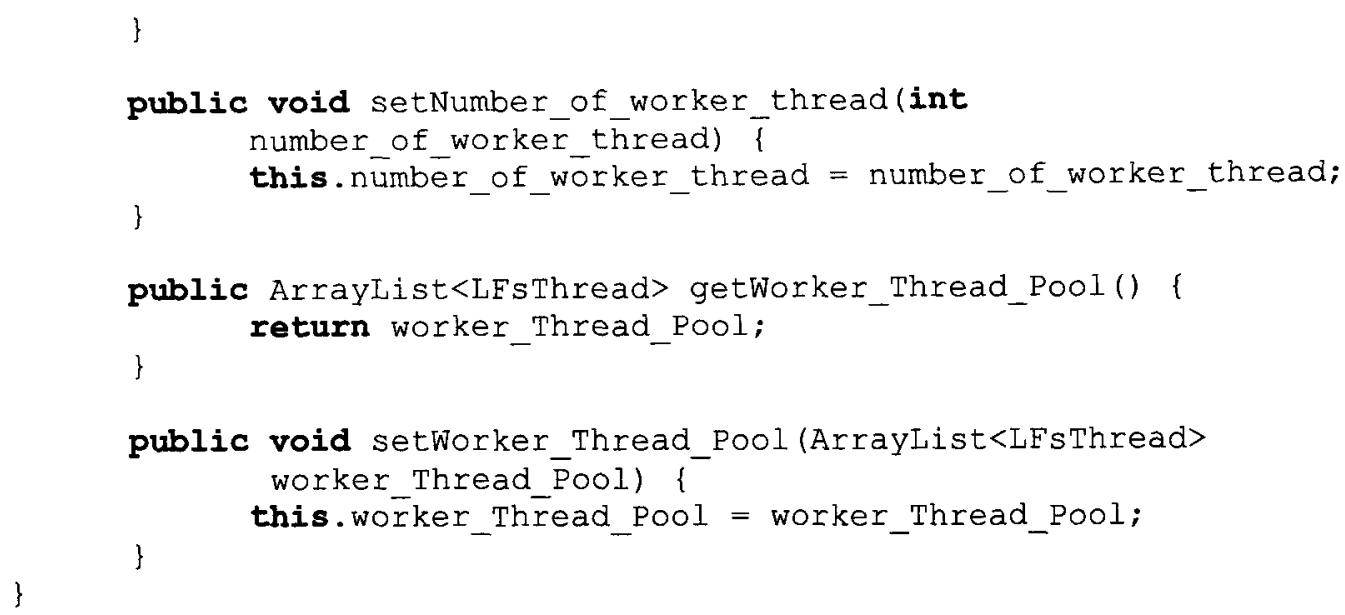

The following code is the LFsThread class being advised by WorkerProcess aspect LFs: public class LFsThread extends Thread f

private int workerThreadId;

private boolean isLeaderThread;

private LFsThreadPool threadPool;

private Ioservice ioservice;

private Datagrampacket workItem = null;

public ServiceTime serviceTime;

private static AtomicInteger key = new AtomicInteger(); private Loggingservice logging;

private AuthenticationService authenticate;

private Authorizationservice authorize;

public LFsThread(int theworkerThreadId)

\{

this. isteaderThread = false;

this. ioService = new IOService ();

this. workerThreadId = theworkerThreadId;

serviceTime = new ServiceTime();

logging = new LoggingService();

authenticate = new Authenticationservice();

\}

authorize = new AuthorizationService();






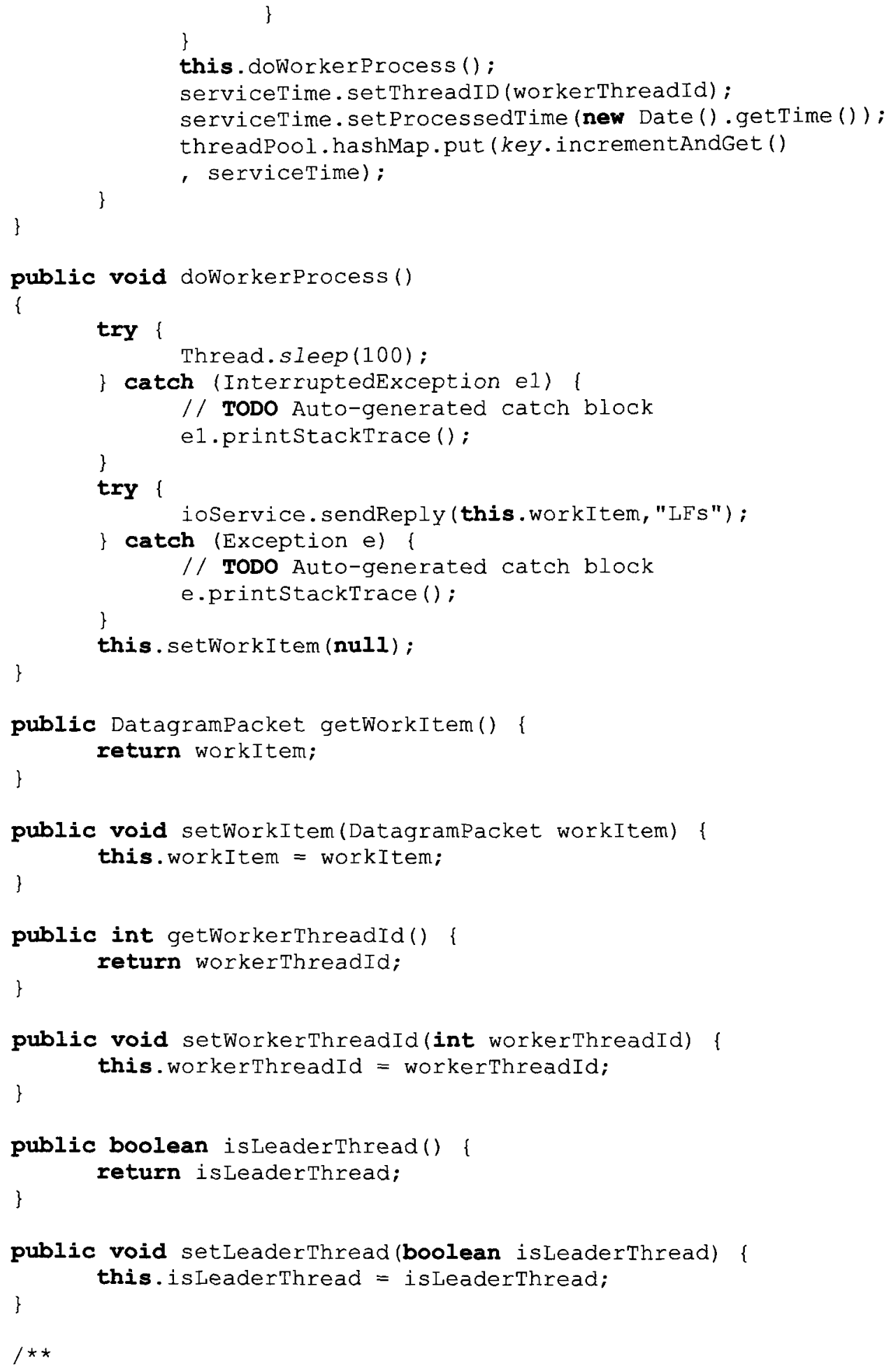




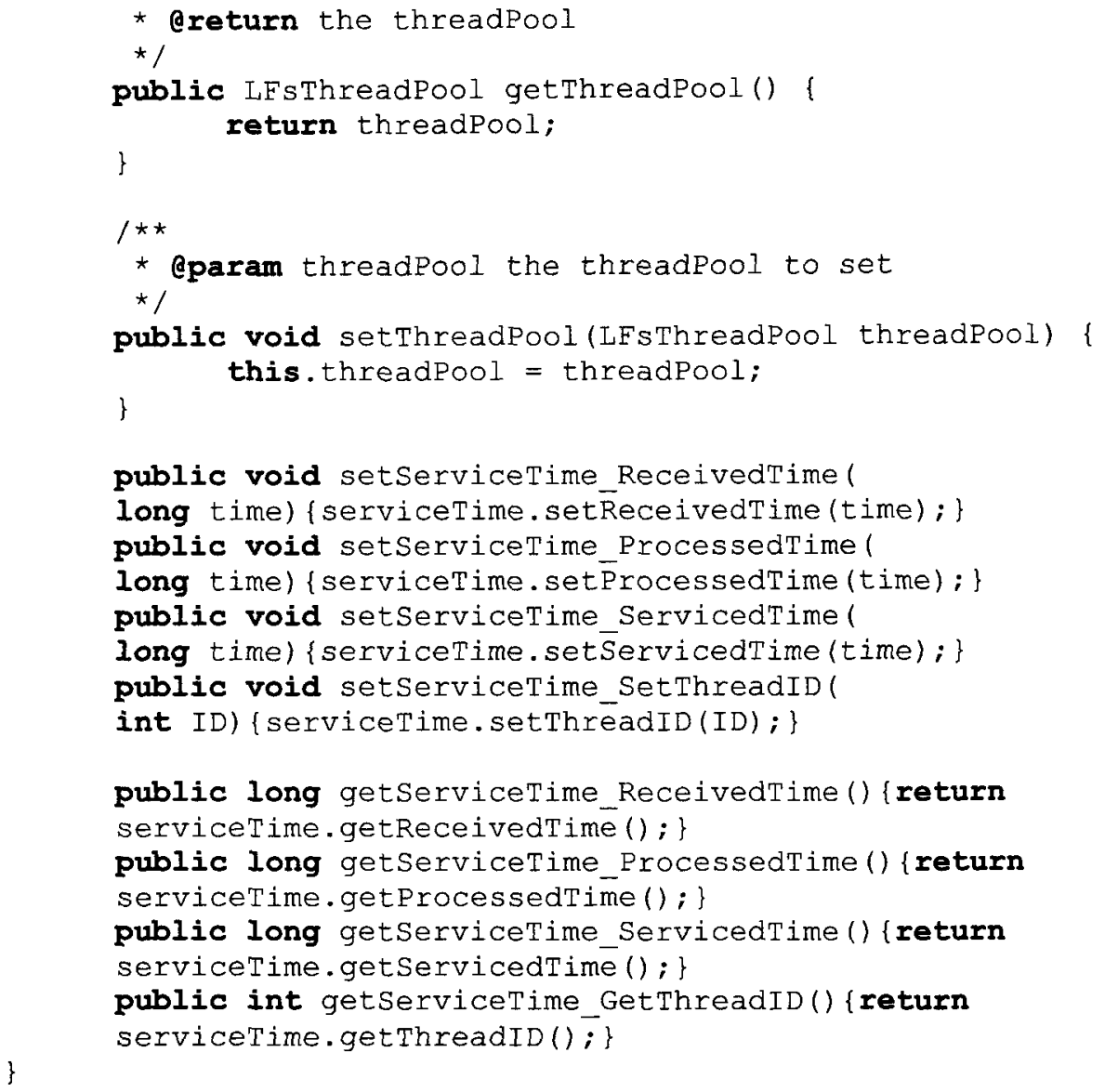

The following code is the public method doLeaderProcess from $L F$ SThreadPool class without advice: public synchronized void doLeaderProcess (LFsThread leader Thread) 


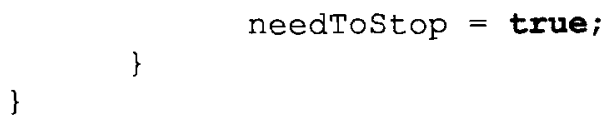

The following code is the public method doWorkerProcess from $L F$ sThread class without advice: public void doworkerprocess () \{

this.setPriority (Thread. NORM PRIORITY);

try

Thread.sleep (100);

\} catch (InterruptedException el) \{

// TODO Auto-generated catch block

e1.printStackTrace ();

\}

try

ioService.sendReply (this.workItem, "LFs");

\} catch (Exception e) \{

// TODo Auto-generated catch block

e.printstackTrace ();

?

\}

this.setWorkItem (null); 


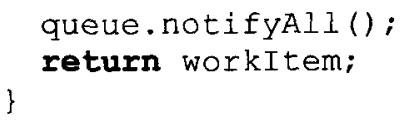

The following code is the AsyncThread class being advised by writeMessageQueue aspect LFs:

public class Asyncthread implements Runnable \{

private Messagequeue queue;

private IOService theIOService;

private Node workItem;

private int numPackage, count;

private Thread parentThread;

private ElapsTime elapseTime;

private LoggingService logging;

private Authenticationservice authenticate;

private Authorizationservice authorize;

public Asyncthread(Messagequeue queue, Thread hsHa,

ElapsTime elapseTime) \{

theIoservice = new IOService ();

theIOService.creatReceiveSocket ();

this.queve = queue;

this. parentThread $=$ hsHa;

count $=0$;

this.elapseTime = elapseTime;

logging = new Loggingservice();

authenticate = new Authenticationservice();

authorize = new AuthorizationService();

\}

public void run() \{

numPackage $=32000$;

while (count < numPackage) \&

try 1

workItem = new Node (this.theIoservice

.receiveDatagramPacket (),

((new Date()).getTime()), null);

\} catch (Exception e1) \{

el.printStackTrace();

\}

if (elapseTime.firstPacketArivalTime $=0)$ \{ elapseTime.firstPacketArivalTime = new

\} Date ().getTime();

queue.addIast (workItem.getItem(), workItem.getTime());

\} count++;

parentThread. interrupt (); 
The following code is the SyncThread class being advised by readMessageQueue aspect LFs: public class SyncThread extends Thread \{

private Messagequeue queue;

private int workerThreadId;

private IOService ioService;

static int numPackageReceived;

private ConcurrentHashMap<Integer, ServiceTime> hashMap;

private static AtomicInteger key = new AtomicInteger();

private Loggingservice logging;

private Authenticationservice authenticate;

private AuthorizationService authorize;

public SyncThread(Messagequeue queue, int workerThreadId, ConcurrentHashMap<Integer, ServiceTime> map) \{

this.queue = queue;

this. workerThreadId = workerThreadId;

ioservice = new IOService ();

hashMap = map;

logging = new Loggingservice();

authenticate = new Authenticationservice();

\}

authorize = new AuthorizationService();

public void run() (

Node workItem = null;

while (! (Thread.currentThread().isInterrupted() \&\& queue.isEmpty()) 1

workItem = queue. removeFirst ();

if (workItem ! = null) \{

this.processitem (workItem);

\}

\}

\}

public void processitem(Node s) \{

try \{

Thread.sleep (100);

\} catch (InterruptedException el) \{

Thread. current Thread() . interrupt ();

\}

try \{

this.ioService.sendReply (s.getItem(), "HS/HA");

\} catch (Exception e) (

e.printstackTrace ();

\}

long time1, time2;

timel = s.getTime ()$; / /$ received time

time2 = new Date().getTime (); // processed time

synchronized (hashMap) \&

hashMap.put (key.incrementAndGet (), new

ServiceTime(time1, time2)); 


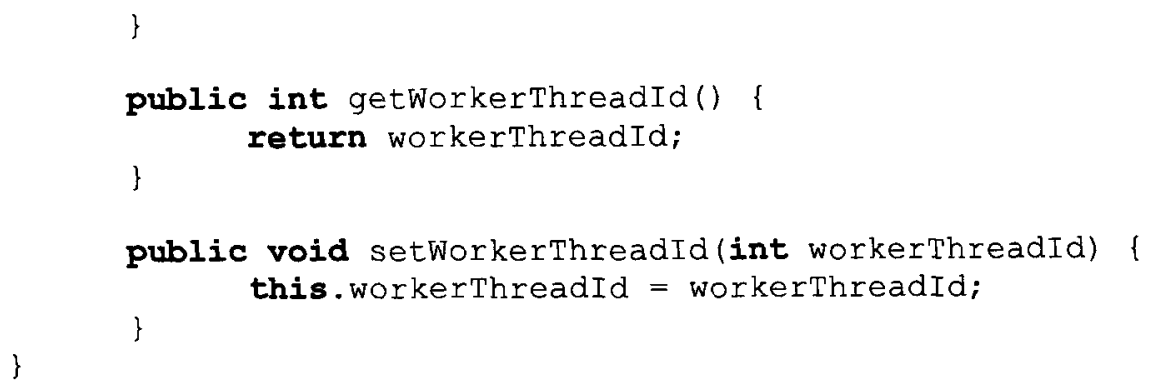

The following code public run method from the AsyncThread class without advice:

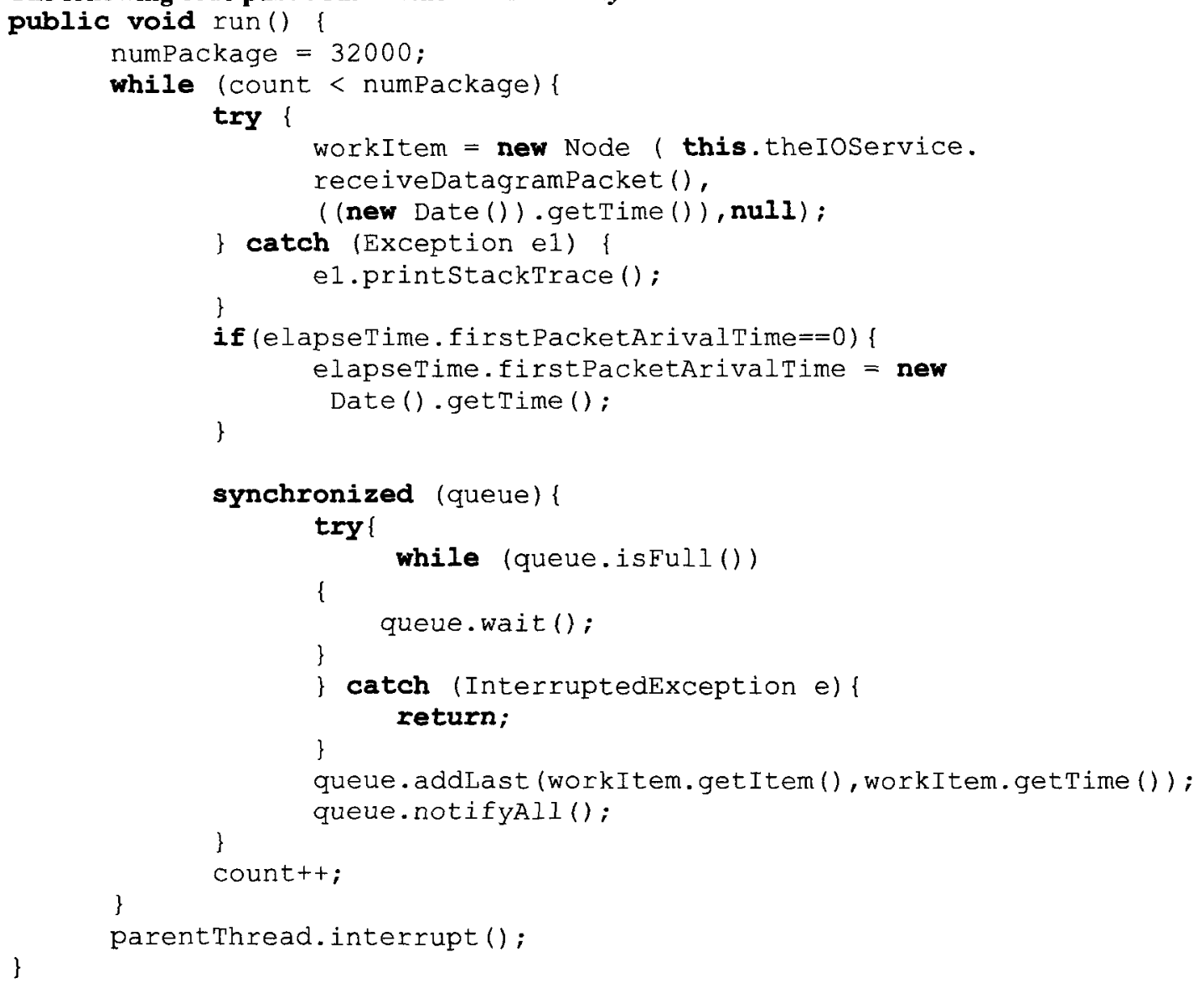

The following code public run method from the SyncThread class without advice: public void run() \{ Node workItem = null;

while (! (Thread.currentThread(). is Interrupted() \&\& queue.isEmpty()) ) ( synchronized (queue) ( 
$\operatorname{try}\{$

while (queve.isEmpty())

queue.wait ();

\} catch (InterruptedException e) \{

Thread. currentThread(). interrupt ();

continue;

\}

workItem = queue. removeFirst ();

queve. notifyAll();

\}

if (workItem ! = null)

this.processitem (workItem);

\} 